\title{
A survey on robotic devices for upper limb rehabilitation
}

\author{
Paweł Maciejasz ${ }^{1,2,3}$, Jörg Eschweiler ${ }^{4 *}$, Kurt Gerlach-Hahn $^{5}$, Arne Jansen-Troy ${ }^{4}$ and Steffen Leonhardt ${ }^{5}$
}

\begin{abstract}
The existing shortage of therapists and caregivers assisting physically disabled individuals at home is expected to increase and become serious problem in the near future. The patient population needing physical rehabilitation of the upper extremity is also constantly increasing. Robotic devices have the potential to address this problem as noted by the results of recent research studies. However, the availability of these devices in clinical settings is limited, leaving plenty of room for improvement. The purpose of this paper is to document a review of robotic devices for upper limb rehabilitation including those in developing phase in order to provide a comprehensive reference about existing solutions and facilitate the development of new and improved devices. In particular the following issues are discussed: application field, target group, type of assistance, mechanical design, control strategy and clinical evaluation. This paper also includes a comprehensive, tabulated comparison of technical solutions implemented in various systems.
\end{abstract}

\section{Introduction}

An individual's capacity to move is necessary to perform basic activities of daily living (ADL). Movement disorders significantly reduce a patient's quality of life. Disorders of the upper extremities specifically limit the independence of affected subjects. Fortunately, there are various approaches to restore the functionality of the upper extremity, e.g., orthoses, functional electrical stimulation, and physical therapy. Positive outcome of physical rehabilitation, in the case of neurologically based disorders, depends heavily on: onset, duration, intensity and taskorientation of the training [1,2], as well as the patient's health condition, attention and effort [3]. Intense repetitions of coordinated motor activities constitute a significant burden for the therapists assisting patients. In addition and due to economical reasons, the duration of primary rehabilitation is getting shorter and shorter [4]. These problems will probably exacerbate in the future as life expectancy continues to increase accompanied by the prevalence of both moderate and severe motor disabilities in the elderly population [5] and consequently increasing their need of physical assistance. To counteract

\footnotetext{
*Correspondence: eschweiler@hia.rwth-aachen.de

${ }^{4}$ Chair of Medical Engineering (mediTEC), Helmholtz-Institute for Biomedical Engineering, RWTH Aachen University, Pauwelsstraße 20, 52074 Aachen, Germany

Full list of author information is available at the end of the article
}

these problems, prevailing research studies present a wide variety of devices specifically assisting physical rehabilitation. Robotic devices with the ability to perform repetitive tasks on patients are among these technically advanced devices. In fact, robotic devices are already used in clinical practice as well as clinical evaluation. However, considering the number of devices described in the literature, so far only a few of them have succeeded to target the subject group - for more details see Table 1 . Furthermore, it seems that the outcome of the use of devices already in clinical practice is not as positive as expected [3]. New solutions are being considered. Most of the literature reviews on robotic devices for upper extremity rehabilitation (e.g. [6,7]) concentrate on devices that have already undergone clinical evaluation. Gopura and Kiguchi [8] compared the mechanical design of selected robotic devices for upper extremity rehabilitation. However, no other publication presents a summary of different robotic solutions for upper extremity rehabilitation, including those being in the development phase. An assessment of different technical solutions would provide developers of robotic devices for upper limb rehabilitation an evaluation of solutions that have already been considered, and thus learn from successes as well as shortfalls from other research teams. Hence, a comparison of various robotic devices would facilitate the development of 
Table 1 Robotic devices for upper limb rehabilitation

\begin{tabular}{|c|c|c|c|c|c|c|}
\hline $\begin{array}{l}\text { System } \\
\text { name,references }\end{array}$ & DOF & $\begin{array}{l}\text { Supported } \\
\text { movements }\end{array}$ & $\begin{array}{l}\text { Main } \\
\text { control inputs }\end{array}$ & Actuators & $\begin{array}{l}\text { Type; field of } \\
\text { application }\end{array}$ & $\begin{array}{l}\text { Stage of development; } \\
\text { additional information }\end{array}$ \\
\hline \multicolumn{7}{|c|}{ Systems assisting shoulder movements } \\
\hline Kiguchi [114] & 2 & Shoulder - FE, AA & sEMG & DC motors (x2) & $\begin{array}{l}\text { Stationary system } \\
\text { (exoskeleton-based); } \\
\text { power assistance }\end{array}$ & CO study: 1 hs \\
\hline \multicolumn{7}{|c|}{ Systems assisting elbow movements } \\
\hline Cheng [9] & 1 & Elbow - FE & sEMG & DC motor & $\begin{array}{l}\text { Stationary system; } \\
\text { physical therapy }\end{array}$ & Cl study: 5 stroke +5 hs \\
\hline Cozens [10] & 1 & Elbow - FE & Joint angle & $\begin{array}{l}\text { Electric servo- } \\
\text { motor }\end{array}$ & $\begin{array}{l}\text { Stationary system; } \\
\text { physical therapy }\end{array}$ & $\mathrm{Cl}$ study: 10 stroke $+\mathrm{MS}$ \\
\hline Kiguchi [170] & 1 & Elbow - FE & sEMG & DC motor & $\begin{array}{l}\text { Stationary system } \\
\text { (exoskeleton-based); } \\
\text { physical therapy }\end{array}$ & Co study: 2 hs \\
\hline $\begin{array}{l}\text { MARIONET, Sulzer } \\
{[142]}\end{array}$ & 1 & Elbow - FE & Joint angle & $\begin{array}{l}\text { AC servomotor } \\
\text { (SEA) }\end{array}$ & $\begin{array}{l}\text { Stationary system } \\
\text { (end-effector-based); } \\
\text { physical therapy }\end{array}$ & C0 study: 6 hs \\
\hline Mavroidis [11] & 1 & Elbow - FE & Force/torque & DC motor & $\begin{array}{l}\text { Portable orthosis } \\
\text { (continuous passive } \\
\text { motion device); } \\
\text { physical therapy }\end{array}$ & Prototype \\
\hline MEM-MRB, Oda [104] & [1] & [Elbow - flexion] & $\begin{array}{l}\text { Joint angular } \\
\text { velocity, torque }\end{array}$ & MRF brake & $\begin{array}{l}\text { Stationary system; } \\
\text { physical therapy }\end{array}$ & CO study: 1 hs \\
\hline $\begin{array}{l}\text { Myomo e100, Myomo, } \\
\text { Inc.; Stein [172] }\end{array}$ & 1 & Elbow - FE & sEMG & DC motor (x1) & $\begin{array}{l}\text { Portable orthosis; } \\
\text { physical therapy }\end{array}$ & $\begin{array}{l}\text { Commercial system (FDA } \\
\text { clearance); Cl study: } 8 \mathrm{cS}\end{array}$ \\
\hline Ögce [171] & 1 & Elbow - FE & sEMG & DC step motor & $\begin{array}{l}\text { Wearable shoulder- } \\
\text { elbow orthosis; } \\
\text { physical therapy }\end{array}$ & $\begin{array}{l}\text { Cl study: } 2 \text { traumatic } \\
\text { brachial plexus injury }\end{array}$ \\
\hline Pylatiuk [153] & 1 & Elbow - FE & sEMG & Hydraulic & $\begin{array}{l}\text { Wearable orthosis; } \\
\text { physical therapy }\end{array}$ & First prototype \\
\hline Rosen [169] & 1 & Elbow - FE & sEMG & DC motor $(x 1)$ & $\begin{array}{l}\text { Stationary system } \\
\text { (exoskeleton-based); } \\
\text { power assistance }\end{array}$ & $\begin{array}{l}\text { C0 study: } 1 \text { hs; predecessor } \\
\text { of CADEN-7 }\end{array}$ \\
\hline Song [12] & 1 & Elbow - FE & sEMG & AC servo motor & $\begin{array}{l}\text { Stationary system } \\
\text { (end-effector-based); } \\
\text { physical therapy }\end{array}$ & $\begin{array}{l}\text { Cl studies: } 8 \text { CS [12], } 7 \text { CS } \\
\text { [13], } 3 \text { CS [14] }\end{array}$ \\
\hline Vanderniepen [143] & 1 & Elbow - FE & Joint angle & $\begin{array}{l}\text { Electric motors (x2) } \\
(\mathrm{SEA})\end{array}$ & $\begin{array}{l}\text { Wearable orthosis; } \\
\text { orthopedic physical } \\
\text { therapy }\end{array}$ & Prototype \\
\hline \multicolumn{7}{|c|}{ Systems assisting forearm movements } \\
\hline Kung [15] & 1 & Forearm - PS & $\begin{array}{l}\text { Joint angle, } \\
\text { torque }\end{array}$ & $\begin{array}{ll}\text { AC } & \text { servomotor } \\
(1) & \end{array}$ & $\begin{array}{l}\text { Stationary system; } \\
\text { physical therapy }\end{array}$ & Cl study: 7 cS + 8 hs [16] \\
\hline \multicolumn{7}{|c|}{ Systems assisting wrist movements } \\
\hline ASSIST, Sasaki [146] & 1 & Wrist - flexion & Joint angle & $\begin{array}{l}\text { Rotary-type } \\
\text { pneumatic } \\
\text { actuators (x2) }\end{array}$ & $\begin{array}{l}\text { Wearable orthosis; } \\
\text { power assistance }\end{array}$ & CO study: 5 hs \\
\hline Colombo [17] & 1 & Wrist - FE & Torque & Not specified & $\begin{array}{l}\text { Stationary system; } \\
\text { physical therapy }\end{array}$ & Cll study: 20(8) CS \\
\hline $\mathrm{Hu}[18]$ & 1 & Wrist - FE & sEMG & Electric motor & $\begin{array}{l}\text { Stationary system } \\
\text { (end-effector-based); } \\
\text { physical therapy }\end{array}$ & Cl study: 15 cS \\
\hline Loureiro [100] & [1] & {$[$ Wrist - FE] } & $\begin{array}{l}\text { Hand motion } \\
\text { (tremor) }\end{array}$ & MRF brake & $\begin{array}{l}\text { Wearable orthosis; } \\
\text { tremor suppression }\end{array}$ & Cl study: 1 ET \\
\hline PolyJbot, Song [175] & 1 & Wrist - FE & $\begin{array}{l}\text { sEMG, joint } \\
\text { angle and } \\
\text { torque }\end{array}$ & DC servomotor $(x 1)$ & $\begin{array}{l}\text { Stationary system; } \\
\text { physical therapy }\end{array}$ & Cll study: 27(15) cS [19] \\
\hline \multicolumn{7}{|c|}{ Systems assisting finger(s) movements } \\
\hline $\begin{array}{l}\text { Amadeo, tyromotion } \\
\text { GmbH }\end{array}$ & 5 & $\begin{array}{l}\text { Fingers (each) - } \\
\text { FE }\end{array}$ & $\begin{array}{l}\text { End-point } \\
\text { position } \\
\text { and force }\end{array}$ & Electric motors & $\begin{array}{l}\text { Stationary system } \\
\text { (end-effector-based); } \\
\text { physical therapy }\end{array}$ & $\begin{array}{l}\text { Commercial system; Cl } \\
\text { study: } 7 \text { aS [20] }\end{array}$ \\
\hline
\end{tabular}


Table 1 Robotic devices for upper limb rehabilitation (Continued)

\begin{tabular}{|c|c|c|c|c|c|c|}
\hline Chen [21] & 5 & $\begin{array}{l}\text { Independent } \\
\text { linear movement } \\
\text { of each finger }\end{array}$ & $\begin{array}{l}\text { Fingers } \\
\text { positions and } \\
\text { forces, sEMG }\end{array}$ & $\begin{array}{l}\text { DC linear motors } \\
(\times 5)\end{array}$ & $\begin{array}{l}\text { Stationary system } \\
\text { (end-effector-based); } \\
\text { physical therapy }\end{array}$ & Co study: 1 hs \\
\hline $\begin{array}{l}\text { CyberGrasp, } \\
\text { CyberGlove Systems } \\
\text { LLC; Turner [22] }\end{array}$ & {$[5]$} & $\begin{array}{l}\text { [Resistive force to } \\
\text { each finger] }\end{array}$ & $\begin{array}{l}\text { Joint angles } \\
\text { (CyberGlove) }\end{array}$ & DC motors (x5) & $\begin{array}{l}\text { Force-feedback } \\
\text { glove; interactions } \\
\text { with virtual } \\
\text { environment }\end{array}$ & $\begin{array}{l}\text { Commercial system for } \\
\text { other applications, used in } \\
\text { some clinical studies e.g. } \\
{[191,192]}\end{array}$ \\
\hline Ertas [23] & 1 & $\begin{array}{l}\text { Concurrent FE of } \\
3 \text { joints of a single } \\
\text { finger }\end{array}$ & Joint angles & DC motor $(x 1)$ & $\begin{array}{l}\text { Finger exoskeleton } \\
\text { (underactuated } \\
\text { mechanism); tendon } \\
\text { physical therapy }\end{array}$ & Co study: 4 hs \\
\hline Fuxiang [24] & 4 & $\begin{array}{l}\text { Index finger- FE } \\
(x 3), A A\end{array}$ & $\begin{array}{l}\text { Joint positions } \\
\text { and toques }\end{array}$ & $\begin{array}{l}\text { Linear stepping } \\
\text { motors }\end{array}$ & $\begin{array}{l}\text { Modular-finger } \\
\text { exoskeleton } \\
\text { (continuous passive } \\
\text { motion device); } \\
\text { physical therapy }\end{array}$ & Co study: 3 hs \\
\hline Gloreha, Idrogenet srl & 5 & $\begin{array}{l}\text { Independent } \\
\text { passive movement } \\
\text { of each finger }\end{array}$ & $\begin{array}{l}\text { Fingers } \\
\text { positions }\end{array}$ & Electric motors (x5) & $\begin{array}{l}\text { Portable (Gloreha } \\
\text { Lite)/Movable } \\
\text { (Gloreha } \\
\text { Professional) (end- } \\
\text { effector-based, } \\
\text { cable-driven); } \\
\text { physical therapy }\end{array}$ & $\begin{array}{l}\text { Commercial system (CE } \\
\text { mark); Cll study: } 10(5) \text { sS } \\
\text { [25], Cl studies: } 9 \text { stroke }+ \\
3 \text { other diseases [26], } 4 \text { cS } \\
\text { [27] }\end{array}$ \\
\hline $\begin{array}{l}\text { Hand of Hope, } \\
\text { Rehab-Robotics } \\
\text { Comp. Ltd., Ho [28] }\end{array}$ & 5 & $\begin{array}{l}\text { Each finger } \\
\text { separately - FE }\end{array}$ & sEMG & $\begin{array}{l}\text { DC linear motors } \\
(\times 5)\end{array}$ & $\begin{array}{l}\text { Portable system } \\
\text { (orthosis); } \\
\text { physical therapy }\end{array}$ & $\begin{array}{l}\text { Commercial system (CE } \\
\text { Mark), Cl study: } 8 \mathrm{cS}\end{array}$ \\
\hline $\begin{array}{l}\text { HandCARE, Dovat } \\
\text { [113] }\end{array}$ & 5 & $\begin{array}{l}\text { Independent } \\
\text { linear movement } \\
\text { of each finger (1 } \\
\text { at a time) }\end{array}$ & $\begin{array}{l}\text { Fingers } \\
\text { positions and } \\
\text { forces }\end{array}$ & DC motor $(x 1 !)$ & $\begin{array}{l}\text { Stationary system } \\
\text { (end-effector- } \\
\text { based, cable-driven); } \\
\text { physical therapy }\end{array}$ & Cl study: $5 \mathrm{cS}+8 \mathrm{hs}$ \\
\hline $\begin{array}{l}\text { HEXORR, Schabowsky } \\
\text { [29] }\end{array}$ & 2 & $\begin{array}{l}\text { Thumb - FE, } \\
\text { other fingers } \\
\text { together - FE }\end{array}$ & $\begin{array}{l}\text { Fingers } \\
\text { positions and } \\
\text { forces }\end{array}$ & $\begin{array}{l}\text { DC motor }(x 1), A C \\
\text { motor }(x 1)\end{array}$ & $\begin{array}{l}\text { Stationary system } \\
\text { (end-effector- } \\
\text { based, cable-driven); } \\
\text { physical therapy }\end{array}$ & Cl study: $5 \mathrm{cS}+9$ hs \\
\hline HIFE, Mali [183] & 2 & 1 finger - FE & $\begin{array}{l}\text { End-point } \\
\text { position }\end{array}$ & DC motors & $\begin{array}{l}\text { Haptic interface } \\
\text { (end-effector-based); } \\
\text { physical therapy }\end{array}$ & Prototype \\
\hline $\begin{array}{l}\text { InMotion HAND, } \\
\text { previous name } \\
\text { InMotion 5.0, } \\
\text { Interactive Motion } \\
\text { Tech., Inc.; Masia [165] }\end{array}$ & 1 & $\begin{array}{l}\text { All fingers } \\
\text { together - GR }\end{array}$ & Not specified & DC brushless motor & $\begin{array}{l}\text { Add-on module for } \\
\text { InMotion ARM; } \\
\text { physical therapy }\end{array}$ & Commercial system \\
\hline Kline [30] & 1 & $\begin{array}{l}\text { All fingers } \\
\text { together - } \\
\text { extension }\end{array}$ & $\begin{array}{l}\text { Joint angles, } \\
\text { sEMG }\end{array}$ & Pneumatic & $\begin{array}{l}\text { Wearable glove; } \\
\text { physical therapy }\end{array}$ & Cl study: 1 stroke + hs (np) \\
\hline Lucas [147] & 1 & $\begin{array}{l}\text { Index finger - } \\
\text { flexion (passive } \\
\text { extension) }\end{array}$ & sEMG & Pneumatic (x2) & $\begin{array}{l}\text { Wearable orthosis; } \\
\text { grasp assistance }\end{array}$ & Cl study: $1 \mathrm{SCl}$ \\
\hline $\begin{array}{l}\text { MR_CHIROD v.2, } \\
\text { Khanicheh [158] }\end{array}$ & {$[1]$} & $\begin{array}{l}\text { [All fingers } \\
\text { together - GR] }\end{array}$ & $\begin{array}{l}\text { Finger position } \\
\text { and torque }\end{array}$ & ERF brake & $\begin{array}{l}\text { Exercising device } \\
\text { (handle-like); physical } \\
\text { therapy }\end{array}$ & $\begin{array}{l}\text { Co study: hs (np); } \\
\text { fMRI compatible }\end{array}$ \\
\hline MRAGES, Winter [157] & {$[5]$} & [Fingers (each) - FE] & $\begin{array}{l}\text { Finger positions } \\
\text { and torques }\end{array}$ & MRF brakes (5) & $\begin{array}{l}\text { Force-feedback } \\
\text { glove; physical } \\
\text { therapy }\end{array}$ & Prototype \\
\hline Mulas [31] & 2 & $\begin{array}{l}\text { Thumb - FE, } \\
\text { other fingers } \\
\text { together - FE }\end{array}$ & $\begin{array}{l}\text { sEMG, pulleys } \\
\text { position }\end{array}$ & $\begin{array}{l}\text { DC servo motors } \\
(x 2)\end{array}$ & $\begin{array}{l}\text { Wearable orthosis; } \\
\text { physical therapy }\end{array}$ & Cl study: 1 sS \\
\hline Nathan [167] & 1 & $\begin{array}{l}\text { All fingers } \\
\text { together - grasp } \\
\text { (passive release) }\end{array}$ & $\begin{array}{l}\text { Hand-held } \\
\text { trigger, index and } \\
\text { thumb fingers } \\
\text { joint angles }\end{array}$ & FES & $\begin{array}{l}\text { Wearable orthosis } \\
\text { (glove); physical } \\
\text { therapy }\end{array}$ & Cl study: 2 stroke +1 hs \\
\hline $\begin{array}{l}\text { PowerGrip, Broaden } \\
\text { Horizons, Inc. }\end{array}$ & 1 & $\begin{array}{l}\text { Thumb, index } \\
\text { and middle finger }\end{array}$ & $\begin{array}{l}\text { Switches } \\
\text { or sEMG }\end{array}$ & DC motor (1) & $\begin{array}{l}\text { Wearable orthosis; } \\
\text { grasp assistance }\end{array}$ & Commercial system \\
\hline
\end{tabular}


Table 1 Robotic devices for upper limb rehabilitation (Continued)

\begin{tabular}{|c|c|c|c|c|c|c|}
\hline $\begin{array}{l}\text { Reha-Digit, } \\
\text { Reha-Stim; } \\
\text { Hesse [32] }\end{array}$ & 1 & $\begin{array}{l}4 \text { fingers (except } \\
\text { the thumb) } \\
\text { together - FE }\end{array}$ & None & DC motor & $\begin{array}{l}\text { Portable system } \\
\text { (rotating handle); } \\
\text { physical therapy }\end{array}$ & $\begin{array}{l}\text { Commercial system (CE } \\
\text { mark); CIl study: 8(4) sS, } \\
\text { Cl study: } 1 \text { cS }\end{array}$ \\
\hline Rosati [144] & 1 & $\begin{array}{l}4 \text { fingers (except } \\
\text { the thumb) } \\
\text { together - FE }\end{array}$ & $\begin{array}{l}\text { Not } \\
\text { selected yet }\end{array}$ & DC motor (SEA) & $\begin{array}{l}\text { Wearable orthosis; } \\
\text { physical therapy }\end{array}$ & Design \\
\hline Rotella [33] & 4 & $\begin{array}{l}\text { Index finger } \\
\text { flexion (x2) } \\
\text { (passive } \\
\text { extension), } \\
\text { thumb - } \\
\text { flexion, other } \\
\text { fingers together - } \\
\text { flexion }\end{array}$ & Not specified & Electric motors & $\begin{array}{l}\text { Wearable orthosis; } \\
\text { grasp assistance }\end{array}$ & Design \\
\hline $\begin{array}{l}\text { Rutgers Master II-ND, } \\
\text { Bouzit [184] }\end{array}$ & 4 & $\begin{array}{l}\text { Thumb, index, } \\
\text { middle, and ring } \\
\text { finger - FE }\end{array}$ & $\begin{array}{l}\text { Actuator } \\
\text { translation and } \\
\text { inclination }\end{array}$ & Pneumatic (x4) & $\begin{array}{l}\text { Force-feedback } \\
\text { glove; interactions } \\
\text { with virtual } \\
\text { environment }\end{array}$ & $\begin{array}{l}\text { Research device; } \\
\text { often used for } \\
\text { hand therapy } \\
\text { (e.g. [185-187]) }\end{array}$ \\
\hline $\begin{array}{l}\text { Salford Hand } \\
\text { Exoskeleton, } \\
\text { Sarakoglou [34] }\end{array}$ & 7 & $\begin{array}{l}\text { Index, middle, and } \\
\text { ring finger }-F E \\
(x 2) \text {, thumb }-F E\end{array}$ & $\begin{array}{l}\text { Joint angles } \\
\text { and end-point } \\
\text { force }\end{array}$ & DC motors & $\begin{array}{l}\text { Wearable orthosis } \\
\text { (exoskeleton); } \\
\text { physical therapy }\end{array}$ & C0 study: hs (np) \\
\hline Tong [35] & 10 & $\begin{array}{l}\text { Each finger - FE } \\
(x 2)\end{array}$ & sEMG & $\begin{array}{l}\text { Electric linear } \\
\text { motors }(x 10)\end{array}$ & $\begin{array}{l}\text { Portable system } \\
\text { (wearable orthosis); } \\
\text { physical therapy }\end{array}$ & Cl study: 2 cS \\
\hline $\begin{array}{l}\text { TU Berlin Finger } \\
\text { Exoskeleton, Wege [36] }\end{array}$ & 4 & $\begin{array}{l}1 \text { finger - FE (x3), } \\
\text { AA }\end{array}$ & Joint angles & DC motors (x4) & $\begin{array}{l}\text { Finger exoskeleton; } \\
\text { physical therapy }\end{array}$ & CO study: 1 hs \\
\hline $\begin{array}{l}\text { TU Berlin Hand } \\
\text { Exoskeleton, Fleischer } \\
{[117]}\end{array}$ & 20 & $\begin{array}{l}\text { FE and AA of all } \\
\text { major joints of } \\
\text { each finger }\end{array}$ & $\begin{array}{l}\text { Joint angles, } \\
\text { end-point force, } \\
\text { sEMG }\end{array}$ & DC motors & $\begin{array}{l}\text { Wearable orthosis } \\
\text { (exoskeleton); } \\
\text { physical therapy }\end{array}$ & Prototype \\
\hline Worsnopp [37] & 3 & $\begin{array}{l}\text { Index finger - } \\
\text { FE (x3) }\end{array}$ & $\begin{array}{l}\text { Joint angles } \\
\text { and torques }\end{array}$ & $\begin{array}{l}\text { DC brushless ser- } \\
\text { vomotors }(x 6)\end{array}$ & $\begin{array}{l}\text { Finger exoskeleton; } \\
\text { physical therapy }\end{array}$ & Prototype \\
\hline Xing [38] & 2 & $\begin{array}{l}\text { Thumb-FE, } \\
\text { other fingers } \\
\text { together - FE }\end{array}$ & Position, force & $\begin{array}{l}\text { Pneumatic } \\
\text { (PAMs) }(\times 2)\end{array}$ & $\begin{array}{l}\text { Wearable orthosis; } \\
\text { physical therapy }\end{array}$ & CO study: 3 hs \\
\hline
\end{tabular}

\section{Systems assisting shoulder and elbow movements}

\begin{tabular}{|c|c|c|c|c|c|c|}
\hline ACRE, Schoone [108] & 5 & Shoulder* elbow & Joint angles & $\begin{array}{l}\text { Electrical motors } \\
(\times 5)\end{array}$ & $\begin{array}{l}\text { Stationary system } \\
\text { (end-effector-based); } \\
\text { physical } \\
\text { therapy }\end{array}$ & Cl: 10 sS \\
\hline$A_{C T}{ }^{3 D}$, Ellis [39] & 3 & Shoulder * elbow & $\begin{array}{l}\text { End-point } \\
\text { torque, } \\
\text { position and } \\
\text { velocity } \\
\text { (HapticMaster) }\end{array}$ & $\begin{array}{l}\text { DC brushed } \\
\text { motors } \\
\text { (HapticMaster) }\end{array}$ & $\begin{array}{l}\text { Stationary system } \\
\text { (end-effector-based); } \\
\text { physical therapy } \\
\text { and assessment of } \\
\text { therapy results }\end{array}$ & Cl study: 6 stroke \\
\hline ARC-MIME, Lum [137] & $1+[2]$ & $\begin{array}{l}\text { Shoulder * elbow } \\
\text { (longitudinal } \\
\text { movements of } \\
\text { the forearm) } \\
\text { [forearm's eleva- } \\
\text { tion and yaw] }\end{array}$ & $\begin{array}{l}\text { Forearm } \\
\text { position and } \\
\text { torque }\end{array}$ & $\begin{array}{l}\text { DC motor }(x 1) \text {, } \\
\text { magnetic particle } \\
\text { brakes }(x 2)\end{array}$ & $\begin{array}{l}\text { Stationary system } \\
\text { (end-effector-based); } \\
\text { physical } \\
\text { therapy }\end{array}$ & $\begin{array}{l}\text { An attempt to } \\
\text { commercialize; Cl study: } 4 \\
\text { cS; merges concepts from } \\
\text { MIME and ARM Guide }\end{array}$ \\
\hline $\begin{array}{l}\text { ARM Guide, } \\
\text { Reinkensmeyer [136] }\end{array}$ & $1+[2]$ & $\begin{array}{l}\text { Shoulder * elbow } \\
\text { (longitudinal } \\
\text { movements of } \\
\text { the forearm) } \\
\text { [forearm's eleva- } \\
\text { tion and yaw] }\end{array}$ & $\begin{array}{l}\text { Forearm } \\
\text { position and } \\
\text { torque }\end{array}$ & $\begin{array}{l}\text { DC motor }(x 1) \text {, } \\
\text { magnetic particle } \\
\text { brakes }(x 2)\end{array}$ & $\begin{array}{l}\text { Stationary system } \\
\text { (end-effector-based); } \\
\text { physical } \\
\text { therapy }\end{array}$ & $\begin{array}{l}\text { Cll study: 19(10) CS [40]; see } \\
\text { also: ARC-MIME }\end{array}$ \\
\hline BFIAMT, Chang [41] & 2 & $\begin{array}{l}\text { Shoulder * elbow } \\
\text { (bilateral longitu- } \\
\text { dinal movements } \\
\text { of the forearms) }\end{array}$ & $\begin{array}{l}\text { End point posi- } \\
\text { tion and torque }\end{array}$ & $\begin{array}{l}\text { DC servomotor } \\
(\times 2) \text {, magnetic } \\
\text { particle brakes } \\
(\times 2)\end{array}$ & $\begin{array}{l}\text { Stationary system } \\
\text { (end-effector-based); } \\
\text { physical } \\
\text { therapy }\end{array}$ & $\begin{array}{l}\text { Cl study: } 20 \text { cS } \\
{[41]}\end{array}$ \\
\hline BONES, Klein [118] & 4 & $\begin{array}{l}\text { Shoulder - FE, AA, } \\
\text { RT, elbow - FE }\end{array}$ & $\begin{array}{l}\text { Joint angles, } \\
\text { cylinder } \\
\text { pressure }\end{array}$ & Pneumatic (x5) & $\begin{array}{l}\text { Stationary system } \\
\text { (parallel robot + } \\
\text { exoskeleton-based } \\
\text { distal part); physical } \\
\text { therapy }\end{array}$ & $\begin{array}{l}\text { Prototype; see also: } \\
\text { Supinator extender (SUE) }\end{array}$ \\
\hline
\end{tabular}


Table 1 Robotic devices for upper limb rehabilitation (Continued)

\begin{tabular}{|c|c|c|c|c|c|c|}
\hline $\begin{array}{l}\text { Dampace, Stienen } \\
{[154]}\end{array}$ & {$[4]$} & $\begin{array}{l}\text { [Shoulder - FE, } \\
\text { AA, RT, elbow - } \\
\text { FE] }\end{array}$ & $\begin{array}{l}\text { Joint angles } \\
\text { and torques }\end{array}$ & $\begin{array}{l}\text { Hydraulic brake } \\
\text { actuators (SEA) }\end{array}$ & $\begin{array}{l}\text { Stationary system } \\
\text { (exoskeleton-based); } \\
\text { physical therapy }\end{array}$ & $\begin{array}{l}\text { Cl study: stroke (np); } \\
\text { see also Limpact }\end{array}$ \\
\hline Freeman [163] & 2 & $\begin{array}{l}\text { Shoulder * elbow } \\
\text { (in the plane) }\end{array}$ & $\begin{array}{l}\text { Handle torque } \\
\text { and position }\end{array}$ & $\begin{array}{l}\text { DC brusheless } \\
\text { servomotors }(x 2) \\
\text { FES }\end{array}$ & $\begin{array}{l}\text { Stationary system } \\
\text { (end-effector-based); } \\
\text { physical therapy }\end{array}$ & C0 study: $18 \mathrm{hs}$ \\
\hline $\begin{array}{l}\text { InMotion ARM, } \\
\text { previous name } \\
\text { InMotion 2.0, } \\
\text { Interactive Motion } \\
\text { Tech., Inc.; based on: } \\
\text { MIT Manus, Krebs } \\
\text { [107] }\end{array}$ & $2+[1]$ & $\begin{array}{l}\text { Shoulder* } \\
\text { elbow (in the } \\
\text { plane + gravity } \\
\text { compensation) }\end{array}$ & $\begin{array}{l}\text { Joint positions, } \\
\text { angular velocity } \\
\text { and torque }\end{array}$ & $\begin{array}{l}\text { DC brushless } \\
\text { motors }\end{array}$ & $\begin{array}{l}\text { Stationary system } \\
\text { (end-effector-based); } \\
\text { physical therapy }\end{array}$ & $\begin{array}{l}\text { Commercial system, } \\
\text { CIII/CIV studies: } 127(49) \\
\text { [203], CII studies: } 56(30) \text { a } \\
\text { [42], 30(10) aS [43] and } \\
\text { others }\end{array}$ \\
\hline $\mathrm{Ju}[44]$ & 2 & $\begin{array}{l}\text { Shoulder * elbow } \\
\text { (in the plane) }\end{array}$ & $\begin{array}{l}\text { Handle torque } \\
\text { and position }\end{array}$ & AC motors ( $x 2)$ & $\begin{array}{l}\text { Stationary system } \\
\text { (end-effector-based; } \\
\text { physical therapy }\end{array}$ & Cl study: stroke (np) \\
\hline Kiguchi [45] & 3 & $\begin{array}{l}\text { Shoulder-FE, AA, } \\
\text { elbow - FE }\end{array}$ & sEMG & DC motors & $\begin{array}{l}\text { Wheelchair mounted } \\
\text { system (exoskeleton- } \\
\text { based); power } \\
\text { assistance }\end{array}$ & $\begin{array}{l}\text { CO study: hs (np); see } \\
\text { also: shoulder, elbow and } \\
\text { shoulder-elbow-forearm } \\
\text { orthoses developed by } \\
\text { Kiguchi and SUEFUL-7 }\end{array}$ \\
\hline Kobayashi [149] & 4 & $\begin{array}{l}\text { Shoulder - FE, AA, } \\
\text { RT, elbow - FE }\end{array}$ & Joint angle & $\begin{array}{l}\text { Pneumatic (PAMs) } \\
(x 10)\end{array}$ & $\begin{array}{l}\text { Wearable (but not } \\
\text { portable) orthosis } \\
\text { ("muscle suit"); power } \\
\text { assistance }\end{array}$ & C0 study: 5 hs \\
\hline Limpact, Stienen [155] & 4 & $\begin{array}{l}\text { Shoulder - FE, AA, } \\
\text { RT, elbow - FE }\end{array}$ & $\begin{array}{l}\text { Joint angles } \\
\text { and torques }\end{array}$ & $\begin{array}{l}\text { Rotational } \\
\text { hydroelastic } \\
\text { actuator (SEA) }\end{array}$ & $\begin{array}{l}\text { Stationary system } \\
\text { (exoskeleton-based); } \\
\text { physical therapy }\end{array}$ & $\begin{array}{l}\text { Design; based on } \\
\text { Dampace }\end{array}$ \\
\hline MariBot, Rosati [46] & 5 & Shoulder * elbow & Motor positions & $\begin{array}{l}\text { DC frameless } \\
\text { brushless motors }\end{array}$ & $\begin{array}{l}\text { Stationary system } \\
\text { (end-effector-based, } \\
\text { cable-driven robot); } \\
\text { physical therapy }\end{array}$ & $\begin{array}{l}\text { Prototype; successor of } \\
\text { NeReBot }\end{array}$ \\
\hline MEMOS, Micera [132] & 2 & $\begin{array}{l}\text { Shoulder * elbow } \\
\text { (in the plane) }\end{array}$ & $\begin{array}{l}\text { Torque and } \\
\text { handle position }\end{array}$ & DC motors (x2) & $\begin{array}{l}\text { Stationary system } \\
\text { (end-effector-based); } \\
\text { physical therapy }\end{array}$ & $\begin{array}{l}\text { Cll study: 20(12) CS [17], } \\
\text { Cl study: } 18 \text { cS [47] }\end{array}$ \\
\hline MIME, Burgar [120] & 6 & Shoulder * elbow & $\begin{array}{l}\text { Forearm } \\
\text { position, } \\
\text { orientation, } \\
\text { torque }\end{array}$ & $\begin{array}{l}\text { DC brushed } \\
\text { servomotors } \\
\text { (PUMA } 560 \text { robot) }\end{array}$ & $\begin{array}{l}\text { Stationary system } \\
\text { (end-effector-based); } \\
\text { physical therapy }\end{array}$ & $\begin{array}{l}\text { Cll studies: } 27(13) \text { CS } \\
\text { [48] and } 30(24) \text { sS [49], } \\
\text { Cl study: } 13 \text { CS [50]; } \\
\text { see also ARC-MIME }\end{array}$ \\
\hline Moubarak [51] & 4 & $\begin{array}{l}\text { Shoulder-FE, AA, } \\
\text { RT, elbow - FE }\end{array}$ & $\begin{array}{l}\text { Joint position, } \\
\text { velocity and } \\
\text { torques }\end{array}$ & $\begin{array}{l}\text { DC brushless } \\
\text { motors }(x 4)\end{array}$ & $\begin{array}{l}\text { Wheelchair- } \\
\text { mounted system } \\
\text { (exoskeleton-based); } \\
\text { physical therapy }\end{array}$ & Prototype \\
\hline NeReBot, Rosati [111] & 3 & Shoulder * elbow & Motor positions & DC motors (x3) & $\begin{array}{l}\text { Stationary system } \\
\text { (end-effector-based, } \\
\text { cable-driven robot); } \\
\text { physical therapy }\end{array}$ & $\begin{array}{l}\text { Cll studies: } 24(12) \text { sS } \\
{[111], 35 \text { (17) aS [52], } 21(11} \\
\text { sS [53]; predecessor of } \\
\text { MariBot }\end{array}$ \\
\hline REHAROB, Toth [125] & 12 & Shoulder * elbow & $\begin{array}{l}\text { End-point } \\
\text { torques }\end{array}$ & $\begin{array}{l}\text { Electrical motors } \\
\text { (ABB IRB } 140 \text { and IRB } \\
1400 H \text { robots) }\end{array}$ & $\begin{array}{l}\text { Stationary system } \\
(2 \text { modified indus- } \\
\text { trial robots); physical } \\
\text { therapy }\end{array}$ & $\begin{array}{l}\text { Cll study: } 22 \text { (13) stroke + } \\
\text { 8(2) TBI [54], Cl study: } \\
6 \text { cS + 2 sS + } 4 \text { hs [125] }\end{array}$ \\
\hline
\end{tabular}

Systems assisting forearm and wrist movements

\begin{tabular}{|c|c|c|c|c|c|c|}
\hline $\begin{array}{l}\text { Bi-Manu-Track, } \\
\text { Reha-Stim; Hesse [55] }\end{array}$ & 1 & $\begin{array}{l}\text { Forearm - PS } \\
{ }^{*} \text { wrist - FE }\end{array}$ & Not specified & Not specified & $\begin{array}{l}\text { Stationary system } \\
\text { (end-effector-based); } \\
\text { physical therapy }\end{array}$ & $\begin{array}{l}\text { Commercial system, CII } \\
\text { study: } 44 \text { (22) sA [56], CI } \\
\text { study: } 12 \text { cS [55] }\end{array}$ \\
\hline $\begin{array}{l}\text { CRAMER, Spencer } \\
\text { [109] }\end{array}$ & 3 & $\begin{array}{l}\text { Forearm - PS, } \\
\text { wrist - FE, AA }\end{array}$ & $\begin{array}{l}\text { Hand accelera- } \\
\text { tions (Nintendo } \\
\text { Wii console) }\end{array}$ & $\begin{array}{l}\text { Digital servomotors } \\
(x 4)\end{array}$ & $\begin{array}{l}\text { Stationary system } \\
\text { (parallel robot); } \\
\text { physical therapy }\end{array}$ & Prototype \\
\hline $\begin{array}{l}\text { InMotion WRIST, } \\
\text { previous name } \\
\text { InMotion 3.0, } \\
\text { Interactive Motion } \\
\text { Tech., Inc.; Krebs [138] }\end{array}$ & 3 & $\begin{array}{l}\text { Forearm - PS, } \\
\text { wrist - FE *AA }\end{array}$ & Joint angles & $\begin{array}{l}\text { DC brushless } \\
\text { motors }(x 3)\end{array}$ & $\begin{array}{l}\text { Stationary system, } \\
\text { may be used as an } \\
\text { add-on for InMotion } \\
\text { ARM; physical } \\
\text { therapy }\end{array}$ & Commercial system \\
\hline
\end{tabular}


Table 1 Robotic devices for upper limb rehabilitation (Continued)

\begin{tabular}{|c|c|c|c|c|c|c|}
\hline RiceWrist, Gupta [1 19] & 4 & $\begin{array}{l}\text { Forearm - PS, } \\
\text { wrist - FE * AA }\end{array}$ & $\begin{array}{l}\text { Joint angles } \\
\text { and forces }\end{array}$ & $\begin{array}{l}\text { Frameless DC } \\
\text { brushless motors }\end{array}$ & $\begin{array}{l}\text { Wearable orthosis; } \\
\text { physical therapy }\end{array}$ & $\begin{array}{l}\text { Prototype; extension for } \\
\text { MIME, see also: MAHI }\end{array}$ \\
\hline $\begin{array}{l}\text { Supinator extender } \\
\text { (SUE), Allington [57] }\end{array}$ & 2 & $\begin{array}{l}\text { Forearm - PS, } \\
\text { wrist - FE }\end{array}$ & $\begin{array}{l}\text { Joint angles } \\
\text { and forces }\end{array}$ & Pneumatic & $\begin{array}{l}\text { Wearable orthosis; } \\
\text { physical therapy }\end{array}$ & $\begin{array}{l}\text { Cl study: } 8 \text { cS; extension for } \\
\text { BONES and ArmeoSpring }\end{array}$ \\
\hline Takaiwa [110] & 3 & $\begin{array}{l}\text { Forearm - PS, } \\
\text { wrist - FE, AA }\end{array}$ & Torque & Pneumatic (x6) & $\begin{array}{l}\text { Stationary system } \\
\text { (parallel robot); } \\
\text { physical therapy }\end{array}$ & Prototype \\
\hline W-EXOS, Gopura [174] & 3 & $\begin{array}{l}\text { Forearm - PS, } \\
\text { wrist - FE, AA }\end{array}$ & $\begin{array}{l}\text { sEMG, hand } \\
\text { force, forearm } \\
\text { torque }\end{array}$ & DC motors (x3) & $\begin{array}{l}\text { Stationary system } \\
\text { (exoskeleton-based); } \\
\text { power assistance }\end{array}$ & $\begin{array}{l}\text { C0 study: 2hs; see also: } \\
\text { SUEFUL-7 }\end{array}$ \\
\hline \multicolumn{7}{|c|}{ Systems assisting wrist and fingers movements } \\
\hline AMES, Cordo [58] & 1 & $\begin{array}{l}\text { wrist and MCP } \\
\text { joints of } 4 \text { fingers } \\
\text { (coupled } \\
\text { together) }\end{array}$ & $\begin{array}{l}\text { Flexion/Extension } \\
\text { torque, sEMG } \\
\text { (optional) }\end{array}$ & $\begin{array}{l}\text { Electric motor }+ \\
2 \text { vibrators (for } \\
\text { flexor and extensor } \\
\text { tendons) }\end{array}$ & $\begin{array}{l}\text { Stationary system } \\
\text { (with desktop } \\
\text { mounted orthosis), } \\
\text { physical therapy (at } \\
\text { home) }\end{array}$ & $\begin{array}{l}\text { FDA clearance; } C \text { l study: } \\
20(11) \text { cS; a modified } \\
\text { version of the system } \\
\text { is used for ankle } \\
\text { rehabilitation }\end{array}$ \\
\hline $\begin{array}{l}\text { Hand Mentor }{ }^{\mathrm{TM}}, \\
\text { Kinematic Muscles, } \\
\text { Inc.; Koeneman [59] }\end{array}$ & 1 & $\begin{array}{l}\text { Wrist and } 4 \text { fin- } \\
\text { gers (except the } \\
\text { thumb) extension }\end{array}$ & $\begin{array}{l}\text { Wrist angle, } \\
\text { flexion torque }\end{array}$ & $\begin{array}{l}\text { Pneumatic (PAM) } \\
(\mathrm{x} 1)\end{array}$ & $\begin{array}{l}\text { Wearable orthosis; } \\
\text { physical therapy }\end{array}$ & $\begin{array}{l}\text { Commercial system (FDA } \\
\text { Class I Device); Cll study: } \\
21(11) \text { sS [60], CI studies: } \\
1 \text { CS [61], } 1 \text { cS [62] }\end{array}$ \\
\hline $\begin{array}{l}\text { HWARD, Takahashi } \\
\text { [130] }\end{array}$ & 3 & $\begin{array}{l}\text { Wrist - FE, } \\
\text { thumb - FE, } \\
\text { other fingers } \\
\text { together - FE }\end{array}$ & $\begin{array}{l}\text { Joint angles } \\
\text { and torques }\end{array}$ & Pneumatic (x3) & $\begin{array}{l}\text { Stationary system } \\
\text { (with desktop } \\
\text { mounted orthosis); } \\
\text { physical therapy }\end{array}$ & Cll study: 13(13) cS \\
\hline $\begin{array}{l}\text { My Scrivener, Obslap } \\
\text { Reseach, LLC; Palsbo } \\
{[190]}\end{array}$ & 3 & Wrist * fingers & $\begin{array}{l}\text { End-point } \\
\text { position and } \\
\text { torque (Novint } \\
\text { Falcon) }\end{array}$ & $\begin{array}{l}\text { Electric motors } \\
\text { (Novint Falcon) }\end{array}$ & $\begin{array}{l}\text { Stationary system } \\
\text { (end-effector-based, } \\
\text { using haptic device); } \\
\text { fine motor hand } \\
\text { therapy }\end{array}$ & $\begin{array}{l}\text { Cl study: } 18 \text { children with } \\
\text { weak handwriting skills }\end{array}$ \\
\hline
\end{tabular}

\begin{tabular}{|c|c|c|c|c|c|c|}
\hline \multicolumn{7}{|c|}{ Systems assisting shoulder, elbow and forearm movements } \\
\hline$A D L E R$, Johnson [63] & $3+\{3\}$ & $\begin{array}{l}\text { Shoulder * elbow } \\
\text { * forearm }\end{array}$ & $\begin{array}{l}\text { End-point } \\
\text { torque, position } \\
\text { and velocity } \\
\text { (HapticMaster) }\end{array}$ & $\begin{array}{l}\text { DC brushed motors } \\
\text { (HapticMaster) }\end{array}$ & $\begin{array}{l}\text { Stationary system } \\
\text { (end-effector-based); } \\
\text { physical therapy }\end{array}$ & C0 study: 8 hs [64] \\
\hline ARAMIS, Pignolo [65] & $6 \times 2$ & $\begin{array}{l}\text { Shoulder - FE, AA, } \\
\text { RT, elbow - FE, } \\
\text { forearm - PS }\end{array}$ & $\begin{array}{l}\text { Joint angles } \\
\text { and torques }\end{array}$ & $\begin{array}{l}\text { DC brushed } \\
\text { motors (x6 per } \\
\text { exoskeleton) }\end{array}$ & $\begin{array}{l}\text { Stationary system } \\
\text { (2 exoskeletons); } \\
\text { physical therapy }\end{array}$ & Cl study: 14 sS \\
\hline $\begin{array}{l}\text { Gentle/S, } \\
\text { Amirabdollahian [121] }\end{array}$ & $3+\{3\}$ & $\begin{array}{l}\text { Shoulder * elbow } \\
\text { * forearm }\end{array}$ & $\begin{array}{l}\text { End-point } \\
\text { torque, position } \\
\text { and velocity } \\
\text { (HapticMaster) }\end{array}$ & $\begin{array}{l}\text { DC brushed motors } \\
\text { (HapticMaster) }\end{array}$ & $\begin{array}{l}\text { Stationary system } \\
\text { (end-effector-based); } \\
\text { physical therapy }\end{array}$ & $\begin{array}{l}\text { Cll study: } 31(31) \text { sS + cS } \\
\text { [66]; predecessor of } \\
\text { Gentle/G }\end{array}$ \\
\hline iPAM, Culmer [67] & 6 & $\begin{array}{l}\text { Shoulder * elbow } \\
\text { * forearm }\end{array}$ & Joint torques & Pneumatic & $\begin{array}{l}\text { Stationary system } \\
\text { ( } 2 \text { robotic arms); } \\
\text { physical therapy }\end{array}$ & Cl study: $16 \mathrm{cS}$ \\
\hline Kiguchi [68] & 4 & $\begin{array}{l}\text { Shoulder-FE, AA, } \\
\text { elbow - FE, } \\
\text { forearm - AA }\end{array}$ & sEMG & DC motors & $\begin{array}{l}\text { Wheelchair mounted } \\
\text { system (exoskeleton- } \\
\text { based); power assis- } \\
\text { tance }\end{array}$ & $\begin{array}{l}\text { C0 study: } 1 \text { hs; see also: } \\
\text { shoulder, elbow and } \\
\text { shoulder-elbow orthoses } \\
\text { developed by Kiguchi and } \\
\text { SUEFUL-7 }\end{array}$ \\
\hline L-Exos, Frisoli [197] & 4 & $\begin{array}{l}\text { Shoulder - FE, AA, } \\
\text { RT, elbow - FE } \\
\{\text { forearm - PS }\end{array}$ & Joint angles & Electric motors $(x 4)$ & $\begin{array}{l}\text { Stationary system } \\
\text { (exoskeleton-based); } \\
\text { physical therapy }\end{array}$ & Cl study: 9 cS [69] \\
\hline MGA, Carignan [70] & 5 & $\begin{array}{l}\text { Shoulder - FE, AA, } \\
R T, \text { VD, elbow - } \\
F E,\{\text { forearm - PS }\}\end{array}$ & Joint torques & $\begin{array}{l}\text { DC brushless } \\
\text { motors }(x 5)\end{array}$ & $\begin{array}{l}\text { Stationary system } \\
\text { (exoskeleton-based); } \\
\text { physical therapy }\end{array}$ & Prototype \\
\hline MULOS, Johnson [168] & 5 & $\begin{array}{l}\text { Shoulder - FE, } A \text { A, } \\
R T \text {, elbow - FE, } \\
\text { forearm - PS }\end{array}$ & Joystick (4 DOF) & Electric motors (x5) & $\begin{array}{l}\text { Wheelchair- } \\
\text { mounted system } \\
\text { (exoskeleton-based); } \\
\text { power assistance } \\
\text { and physical therapy }\end{array}$ & CO study: 1 hs \\
\hline
\end{tabular}


Table 1 Robotic devices for upper limb rehabilitation (Continued)

\begin{tabular}{|c|c|c|c|c|c|c|}
\hline NJIT-RAVR, Fluet [71] & $3+\{3\}$ & $\begin{array}{l}\text { Shoulder* } \\
\text { elbow* forearm }\end{array}$ & $\begin{array}{l}\text { End-point } \\
\text { torque, position } \\
\text { and velocity } \\
\text { (HapticMaster) }\end{array}$ & $\begin{array}{l}\text { DC brushed motors } \\
\text { (HapticMaster) }\end{array}$ & $\begin{array}{l}\text { Stationary system } \\
\text { (end-effector-based); } \\
\text { physical therapy } \\
\text { of children }\end{array}$ & Cl study: 8 CP \\
\hline $\begin{array}{l}\text { RehabExos, Vertechy } \\
\text { [131] }\end{array}$ & 4 & $\begin{array}{l}\text { Shoulder - FE, AA, } \\
\text { RT, elbow - FE } \\
\text { fforearm - PS }\end{array}$ & Joint torques & $\begin{array}{l}\text { Custom-made } \\
\text { frameless brushless } \\
\text { motor }(\times 3), D C \\
\text { motor }(\times 1)\end{array}$ & $\begin{array}{l}\text { Stationary system } \\
\text { (exoskeleton-based); } \\
\text { physical therapy }\end{array}$ & First prototype \\
\hline \multicolumn{7}{|c|}{ Systems assisting shoulder, elbow and fingers movements } \\
\hline $\begin{array}{l}\text { Pneu-WREX, } \\
\text { Wolbrecht [145] }\end{array}$ & $4+\{1\}$ & $\begin{array}{l}\text { Shoulder - FE, AA, } \\
\text { HD, elbow - FE, } \\
\text { \{fingers - GR\} }\end{array}$ & $\begin{array}{l}\text { Joint angles, } \\
\text { grasp force, } \\
\text { cylinder } \\
\text { pressure }\end{array}$ & Pneumatic (x4) & $\begin{array}{l}\text { Stationary system } \\
\text { (exoskeleton-based); } \\
\text { physical therapy }\end{array}$ & $\begin{array}{l}\text { Cl study: } 11 \text { CS [72]; } \\
\text { see also: T-WREX and } \\
\text { ArmeoSpring }\end{array}$ \\
\hline T-WREX, Sanchez [106] & $\{5\}$ & $\begin{array}{l}\{\text { Shoulder }-F E \\
A A, R T \text {, elbow - } \\
\text { FE, fingers - GR\} }\end{array}$ & $\begin{array}{l}\text { Joint angles, } \\
\text { grasp force }\end{array}$ & None & $\begin{array}{l}\text { Wheelchair mounted } \\
\text { gravity balancing } \\
\text { orthosis; physical } \\
\text { therapy }\end{array}$ & $\begin{array}{l}\text { Cll studies: } 23(11) \text { cS [73], } \\
\text { 28(14) CS [74], Cl studies: } \\
9 \mathrm{CS}+5 \mathrm{CS} \text { (2 studies) [75]; } \\
\text { see also: Pneu-WREX and } \\
\text { ArmeoSpring }\end{array}$ \\
\hline
\end{tabular}

\section{Systems assisting elbow, forearm and wrist movements}

\begin{tabular}{|c|c|c|c|c|c|c|}
\hline Ding [179] & 4 & $\begin{array}{l}\text { Elbow - FE, } \\
\text { forearm - PS, } \\
\text { wrist - FE, AA }\end{array}$ & $\begin{array}{l}\text { Joint angles } \\
\text { (a Motion } \\
\text { Capture System } \\
\text { is used) }\end{array}$ & Pneumatic (x8) & $\begin{array}{l}\text { Wearable (but not } \\
\text { portable) orthosis; } \\
\text { power assistance for } \\
\text { explicitly specified } \\
\text { muscles }\end{array}$ & C0 study: 6 hs \\
\hline MAHI, Gupta [76] & 5 & $\begin{array}{l}\text { Elbow - FE, } \\
\text { forearm - PS, } \\
\text { wrist - FE * AA }\end{array}$ & Joint angles & $\begin{array}{l}\text { Frameless DC } \\
\text { brushless motors }\end{array}$ & $\begin{array}{l}\text { Wearable orthosis } \\
\text { (force-feedback } \\
\text { exoskeleton); } \\
\text { physical therapy }\end{array}$ & $\begin{array}{l}\text { Prototype; extension for } \\
\text { MIME; see also: RiceWrist }\end{array}$ \\
\hline WOTAS, Rocon [99] & [3] & $\begin{array}{l}\text { [Elbow - FE, } \\
\text { forearm - PS, } \\
\text { wrist - FE] }\end{array}$ & $\begin{array}{l}\text { Angular } \\
\text { velocity, } \\
\text { torques }\end{array}$ & DC motors (x3) & $\begin{array}{l}\text { Wearable orthosis; } \\
\text { tremor suppression }\end{array}$ & Cl study: 10 mainly ET \\
\hline
\end{tabular}

\begin{tabular}{|c|c|c|c|c|c|c|}
\hline \multicolumn{7}{|c|}{ Systems assisting forearm, wrist and fingers movements } \\
\hline $\begin{array}{l}\text { Haptic Knob, } \\
\text { Lambercy [77] }\end{array}$ & 2 & $\begin{array}{l}\text { Forearm - } \\
P S^{*} \text { wrist }-F E \\
\text { fingers }-G R\end{array}$ & Position, torque & $\begin{array}{l}\text { DC brushed motors } \\
(x 2)\end{array}$ & $\begin{array}{l}\text { Stationary system } \\
\text { ( } 2 \text { parallelograms); } \\
\text { physical therapy }\end{array}$ & Cl study: 3 cS \\
\hline Hasegawa [98] & 11 & $\begin{array}{l}\text { Forearm - PS, } \\
\text { wrist - FE, AA, } \\
\text { thumb - FE }(x 2) \text {, } \\
\text { index finger - } \\
\text { FE }(x 3) \text {, other } \\
\text { fingers together - } \\
\text { FE }(x 3)\end{array}$ & SEMG & DC motors ( $x 11)$ & $\begin{array}{l}\text { Wearable orthosis; } \\
\text { grasp assistance }\end{array}$ & Co study: 1 hs \\
\hline Kawasaki [178] & 18 & $\begin{array}{l}\text { Forearm - PS, } \\
\text { wrist - FE, thumb - } \\
\text { FE }(\times 3), A A \\
\text { other fingers - } \\
\text { FE }(\times 2), A A\end{array}$ & $\begin{array}{l}\text { Joint angles of } \\
\text { healthy hand }\end{array}$ & Servo motors (x22) & $\begin{array}{l}\text { Stationary system } \\
\text { (exoskeleton-based); } \\
\text { physical therapy }\end{array}$ & Co study: 1 hs \\
\hline Scherer [156] & {$[1]$} & $\begin{array}{l}\text { [Forearm and } \\
\text { fingers twisting } \\
\text { movements* } \\
\text { wrist - FE] }\end{array}$ & Position, torque & $\begin{array}{l}\text { Magnetic particle } \\
\text { brake }\end{array}$ & $\begin{array}{l}\text { Stationary system } \\
\text { (end-effector-based, } \\
\text { rotating handle); } \\
\text { physical therapy }\end{array}$ & $\begin{array}{l}\text { Cl study: } 2 \text { stroke }+ \\
1 \text { MS }\end{array}$ \\
\hline \multicolumn{7}{|c|}{ Systems assisting shoulder, elbow, forearm and wrist movements } \\
\hline $\begin{array}{l}\text { Braccio di Ferro, } \\
\text { Casadio [134] }\end{array}$ & 2 & $\begin{array}{l}\text { Shoulder* } \\
\text { elbow * (forearm)* } \\
\text { wrist (in the } \\
\text { horizonatal or } \\
\text { vertical plane) }\end{array}$ & $\begin{array}{l}\text { Device joint } \\
\text { angles, end- } \\
\text { point force }\end{array}$ & $\begin{array}{l}\text { AC brushless } \\
\text { servomotors (x2) }\end{array}$ & $\begin{array}{l}\text { Stationary system } \\
\text { (end-effector-based); } \\
\text { physical therapy }\end{array}$ & $\begin{array}{l}\text { Cl studies: } 10 \mathrm{cS}+4 \text { hs [78] } \\
7 \mathrm{MS}+9 \text { hs [79], } 11 \mathrm{MS}+ \\
11 \mathrm{hs}[80], 8 \mathrm{MS}[81]\end{array}$ \\
\hline CADEN-7, Perry [97] & $2 \times 7$ & $\begin{array}{l}\text { Shoulder - FE, } \\
\text { AA, RT, elbow - } \\
\text { FE, forearm - PS, } \\
\text { wrist - FE, AA }\end{array}$ & $\begin{array}{l}\text { sEMG, joint } \\
\text { angles, angular } \\
\text { velocities and } \\
\text { forces/torques }\end{array}$ & $\begin{array}{l}\text { DC brushed motors } \\
(2 \times 7)\end{array}$ & $\begin{array}{l}\text { Stationary system } \\
\text { (exoskeleton-based), } \\
2 \text { robotic arms; } \\
\text { power assistance }\end{array}$ & C0 study: 1 hs \\
\hline
\end{tabular}


Table 1 Robotic devices for upper limb rehabilitation (Continued)

\begin{tabular}{|c|c|c|c|c|c|c|}
\hline Denève [82] & 3 & $\begin{array}{l}\text { Shoulder * elbow } \\
{ }^{*}\left(\text { forearm) }{ }^{*} \text { wrist }\right.\end{array}$ & $\begin{array}{l}\text { Device joint } \\
\text { angles, end- } \\
\text { point force }\end{array}$ & $\begin{array}{l}\text { AC brushless } \\
\text { motors }(\times 3)\end{array}$ & $\begin{array}{l}\text { Stationary system } \\
\text { (end-effector-based); } \\
\text { physical therapy }\end{array}$ & Prototype \\
\hline EMUL, Furusho [159] & 3 & $\begin{array}{l}\text { Shoulder* } \\
\text { elbow* (forearm)* } \\
\text { wrist }\end{array}$ & $\begin{array}{l}\text { End-point } \\
\text { position }\end{array}$ & $\begin{array}{l}\text { Electric motors + } \\
\text { ERF clutches }\end{array}$ & $\begin{array}{l}\text { Stationary system } \\
\text { (end-effector-based); } \\
\text { physical therapy }\end{array}$ & $\begin{array}{l}\text { Cl study: } 6 \text { stroke; } \\
\text { predecessor of PLEMO, see } \\
\text { also: Robotherapist }\end{array}$ \\
\hline $\begin{array}{l}\text { ESTEC exoskeleton, } \\
\text { Schiele [115] }\end{array}$ & 9 & $\begin{array}{l}\text { Shoulder - FE, } \\
A A, R T, V D, H D \text {, } \\
\text { elbow - FE, } \\
\text { forearm - PS, } \\
\text { wrist - FE, AA }\end{array}$ & Joint angles & Not selected yet & $\begin{array}{l}\text { Wearable system } \\
\text { (exoskeleton-based); } \\
\text { physical therapy }\end{array}$ & First prototype \\
\hline Furuhashi [83] & 3 & $\begin{array}{l}\text { Shoulder * elbow* } \\
(\text { forearm) * wrist }\end{array}$ & $\begin{array}{l}\text { End-point } \\
\text { torque }\end{array}$ & DC motors $(\times 3)$ & $\begin{array}{l}\text { Stationary system } \\
\text { (end-effector-based); } \\
\text { physical therapy }\end{array}$ & Prototype \\
\hline $\begin{array}{l}\text { Hybrid-PLEMO, Kikuchi } \\
\text { [135] }\end{array}$ & 2 & $\begin{array}{l}\text { Shoulder * elbow* } \\
\text { (forearm)* wrist } \\
\text { (in the adjustable } \\
\text { plane) }\end{array}$ & $\begin{array}{l}\text { Device joint } \\
\text { angles, end- } \\
\text { point force }\end{array}$ & $\begin{array}{l}\text { DC servomotors } \\
(\times 2)+\text { ERF } \\
\text { clutches/brakes } \\
(\times 4)\end{array}$ & $\begin{array}{l}\text { Stationary system } \\
\text { (end-effector-based); } \\
\text { physical therapy }\end{array}$ & $\begin{array}{l}\text { Prototype; based on } \\
\text { PLEMO }\end{array}$ \\
\hline Lam [180] & 2 & $\begin{array}{l}\text { Shoulder * elbow* } \\
\text { (forearm) * wrist } \\
\text { (in the plane) }\end{array}$ & $\begin{array}{l}\text { End-point } \\
\text { position, abnor- } \\
\text { mal trunk posi- } \\
\text { tion detection }\end{array}$ & Not specified & $\begin{array}{l}\text { Stationary system } \\
\text { (end-effector-based); } \\
\text { physical therapy }\end{array}$ & C0 study: 8 hs \\
\hline Li [176] & 5 & $\begin{array}{l}\text { Shoulder - FE, } \\
\text { AA, elbow - FE, } \\
\text { forearm - PS, } \\
\text { wrist - FE }\end{array}$ & $\begin{array}{l}\text { sEMG signals } \\
\text { from not } \\
\text { affected arm }\end{array}$ & $\begin{array}{l}A C(x 3) \text { and } D C(x 2) \\
\text { servo motors }\end{array}$ & $\begin{array}{l}\text { Wearable system } \\
\text { (exoskeleton-based); } \\
\text { physical therapy }\end{array}$ & Prototype \\
\hline MACARM, Beer [112] & 6 & $\begin{array}{l}\text { Shoulder* } \\
\text { elbow* forearm * } \\
\text { wrist }\end{array}$ & $\begin{array}{l}\text { End-point posi- } \\
\text { tion and force }\end{array}$ & Electric motors (x8) & $\begin{array}{l}\text { Stationary system } \\
\text { (end-effector-based, } \\
\text { cable-driven robot); } \\
\text { physical therapy }\end{array}$ & Cl study: 5 cS \\
\hline Mathai [84] & 3 & $\begin{array}{l}\text { Shoulder* } \\
\text { elbow* forearm * } \\
\text { wrist }\end{array}$ & $\begin{array}{l}\text { End-point } \\
\text { torque, position } \\
\text { and velocity } \\
\text { (HapticMaster) }\end{array}$ & $\begin{array}{l}\text { DC brushed motors } \\
\text { (HapticMaster) }\end{array}$ & $\begin{array}{l}\text { Stationary system } \\
\text { (end-effector-based); } \\
\text { physical therapy }\end{array}$ & Cl study: 4 cS \\
\hline $\begin{array}{l}\text { MIME-RiceWrist, Gupta } \\
\text { [119] }\end{array}$ & 10 & $\begin{array}{l}\text { Shoulder* } \\
\text { elbow* forearm * } \\
\text { wrist }\end{array}$ & $\begin{array}{l}\text { See separate } \\
\text { information for } \\
\text { MIME and } \\
\text { RiceWrist } \\
\text { system }\end{array}$ & $\begin{array}{l}\text { See separate } \\
\text { information for } \\
\text { MIME and RiceWrist } \\
\text { system }\end{array}$ & $\begin{array}{l}\text { Stationary system } \\
\text { (robotic arm + } \\
\text { orthosis); physical } \\
\text { therapy }\end{array}$ & Cl study: stroke (np) \\
\hline PLEMO, Kikuchi [105] & {$[2]$} & $\begin{array}{l}\text { [Shoulder* elbow* } \\
\text { (forearm) * wrist] } \\
\text { (in the adjustable } \\
\text { plane) }\end{array}$ & $\begin{array}{l}\text { Device joint } \\
\text { angles, end- } \\
\text { point force }\end{array}$ & ERF brakes & $\begin{array}{l}\text { Stationary system } \\
\text { (end-effector-based); } \\
\text { physical therapy }\end{array}$ & $\begin{array}{l}\text { Cl study: } 6 \text { stroke + } \\
27 \text { hs [85]; successor of } \\
\text { EMUL, predecessor of } \\
\text { Hybrid-PLEMO }\end{array}$ \\
\hline $\begin{array}{l}\text { Robotherapist, } \\
\text { Furusho [160] }\end{array}$ & 6 & $\begin{array}{l}\text { Shoulder * } \\
\text { elbow* forearm * } \\
\text { wrist }\end{array}$ & $\begin{array}{l}\text { End-point } \\
\text { position }\end{array}$ & $\begin{array}{l}\text { Electric motors + } \\
\text { ERF clutches }\end{array}$ & $\begin{array}{l}\text { Stationary system } \\
\text { (end-effector-based); } \\
\text { physical therapy }\end{array}$ & $\begin{array}{l}\text { Prototype; see also: } \\
\text { EMUL }\end{array}$ \\
\hline $\begin{array}{l}\text { RUPERTIV, } \\
\text { Balasubrama- nian } \\
\text { [151] }\end{array}$ & 5 & $\begin{array}{l}\text { Shoulder - AA, } \\
\text { RT, elbow - FE, } \\
\text { forearm - PS, } \\
\text { wrist - FE }\end{array}$ & $\begin{array}{l}\text { Joint torques } \\
\text { and actuators } \\
\text { pressure }\end{array}$ & Pneumatic (PAMs) & $\begin{array}{l}\text { Wearable system } \\
\text { (exoskeleton-based); } \\
\text { physical therapy }\end{array}$ & Cl study: 6 cS [86] \\
\hline $\begin{array}{l}\text { Salford Arm } \\
\text { Rehabilitation } \\
\text { Exoskeleton, } \\
\text { Tsagarakis [148] } \\
\end{array}$ & 7 & $\begin{array}{l}\text { Shoulder - FE, } \\
\text { AA, RT, elbow - } \\
\text { FE, forearm - PS, } \\
\text { wrist - FE, AA }\end{array}$ & $\begin{array}{l}\text { Joint positions } \\
\text { and torques }\end{array}$ & $\begin{array}{l}\text { Linear pneumatic } \\
\text { actuators (PAMs) } \\
(\times 14)\end{array}$ & $\begin{array}{l}\text { Stationary system } \\
\text { (exoskeleton-based); } \\
\text { physical therapy }\end{array}$ & Prototype \\
\hline Sophia-3, Rosati [87] & 2 & $\begin{array}{l}\text { Shoulder * } \\
\text { elbow }{ }^{*}\left(\text { forearm) }{ }^{*}\right. \\
\text { wrist (in the } \\
\text { plane) }\end{array}$ & $\begin{array}{l}\text { End-point posi- } \\
\text { tion and force }\end{array}$ & AC motors & $\begin{array}{l}\text { Stationary system } \\
\text { (end-effector-based, } \\
\text { planar cable-driven } \\
\text { robot); physical } \\
\text { therapy }\end{array}$ & $\begin{array}{l}\text { First prototype; see also: } \\
\text { Sophia-4 }\end{array}$ \\
\hline Sophia-4, Rosati [87] & 2 & $\begin{array}{l}\text { Shoulder * } \\
\text { elbow* (forearm)* } \\
\text { wrist (in the } \\
\text { plane) }\end{array}$ & $\begin{array}{l}\text { End-point } \\
\text { position and } \\
\text { force }\end{array}$ & DC motors & $\begin{array}{l}\text { Stationary system } \\
\text { (end-effector-based, } \\
\text { planar cable-driven } \\
\text { robot); physical } \\
\text { therapy }\end{array}$ & $\begin{array}{l}\text { Prototype; see also: } \\
\text { Sophia-3 }\end{array}$ \\
\hline
\end{tabular}


Table 1 Robotic devices for upper limb rehabilitation (Continued)

\begin{tabular}{|c|c|c|c|c|c|c|}
\hline $\begin{array}{l}\text { SUEFUL-7, } \\
{[166]}\end{array}$ & 7 & $\begin{array}{l}\text { Shoulder - FE, } \\
\text { AA, RT, elbow - } \\
\text { FE, forearm - PS, } \\
\text { wrist - FE, AA }\end{array}$ & $\begin{array}{l}\text { sEMG/joint } \\
\text { forces/torques }\end{array}$ & $\begin{array}{l}\text { DC servo motors } \\
(x 7)\end{array}$ & $\begin{array}{l}\text { Stationary system } \\
\text { (exoskeleton-based); } \\
\text { power assistance }\end{array}$ & $\begin{array}{l}\text { CO study: } 2 \text { hs; shoulder- } \\
\text { elbow orthosis integratec } \\
\text { with W-EXOS system }\end{array}$ \\
\hline Takahashi [182] & 2 & $\begin{array}{l}\text { Shoulder* } \\
\text { elbow* } \\
\text { (forearm)* wrist } \\
\text { (in the plane) }\end{array}$ & $\begin{array}{l}\text { End point posi- } \\
\text { tion }\end{array}$ & $\begin{array}{l}\text { Electric } \\
\text { servomotors (x2) }\end{array}$ & $\begin{array}{l}\text { Stationary system } \\
\text { (end-effector-based); } \\
\text { physical therapy }\end{array}$ & $\begin{array}{l}\text { Cl study: } 5 \text { stroke }+ \\
2 \text { Guillain-Bare syndrome }\end{array}$ \\
\hline Tanaka [88] & 2 & $\begin{array}{l}\text { Shoulder * } \\
\text { elbow* (forearm)* } \\
\text { wrist (in the } \\
\text { plane) }\end{array}$ & $\begin{array}{l}\text { End-point force } \\
\text { and position }\end{array}$ & AC linear motor (x2) & $\begin{array}{l}\text { Stationary system } \\
\text { (end-effector-based); } \\
\text { physical therapy }\end{array}$ & CO study: 6 hs \\
\hline UHD, Oblak [139] & 2 & $\begin{array}{l}3 \text { configurations } \\
\text { possible: 1) } \\
\text { shoulder * elbow, } \\
\text { 2) forearm - PS, } \\
\text { wrist - FE, 3) } \\
\text { forearm - PS, } \\
\text { wrist - AA }\end{array}$ & $\begin{array}{l}\text { Torque and } \\
\text { handle position }\end{array}$ & $\begin{array}{l}\text { DC motors (x2), } \\
\text { (SEA) }\end{array}$ & $\begin{array}{l}\text { Stationary system } \\
\text { (end-effector-based); } \\
\text { physical therapy }\end{array}$ & $\begin{array}{l}\text { Cl study: } 1 \mathrm{cS} \text {; } \\
\text { reconfigurable robot }\end{array}$ \\
\hline Umemura [152] & 7 & $\begin{array}{l}\text { Shoulder - FE, } \\
\text { AA, RT, elbow - } \\
\text { FE, forearm - PS, } \\
\text { wrist - FE, AA }\end{array}$ & $\begin{array}{l}\text { Actuators } \\
\text { pressure }\end{array}$ & Hydraulic & $\begin{array}{l}\text { Stationary system } \\
\text { (end-effector-based); } \\
\text { physical therapy }\end{array}$ & Prototype \\
\hline UMH, Morales [127] & 6 & $\begin{array}{l}\text { Shoulder* } \\
\text { elbow* forearm * } \\
\text { wrist }\end{array}$ & Joint torques & Pneumatic & $\begin{array}{l}\text { Stationary system } \\
\text { (two robotic arms); } \\
\text { physical therapy }\end{array}$ & Co study: hs (np) \\
\hline Xiu-Feng [89] & 2 & $\begin{array}{l}\text { Shoulder* } \\
\text { elbow* } \\
\text { (forearm)* wrist } \\
\text { (in the plane) }\end{array}$ & $\begin{array}{l}\text { Device joint } \\
\text { angles, end- } \\
\text { point force }\end{array}$ & $\begin{array}{l}\text { AC servomotors } \\
(\times 2)\end{array}$ & $\begin{array}{l}\text { Stationary system } \\
\text { (end-effector-based); } \\
\text { physical therapy }\end{array}$ & Cl study: 30 stroke \\
\hline
\end{tabular}

\section{Systems assisting shoulder, elbow, forearm, wrist and finger movements (whole arm)}

\begin{tabular}{|c|c|c|c|c|c|c|}
\hline $\begin{array}{l}\text { ArmeoPower, Hocoma } \\
\text { AG; based on: ARMin } \\
\text { III, Nef [90] }\end{array}$ & $6\{+1\}$ & $\begin{array}{l}\text { Shoulder - FE, } \\
\text { AA, RT, elbow - } \\
\text { FE, forearm - } \\
\text { PS, wrist - FE, } \\
\text { \{fingers - GR\} }\end{array}$ & $\begin{array}{l}\text { Joint angles, } \\
\text { grasp force }\end{array}$ & DC motors $(x 6)$ & $\begin{array}{l}\text { Stationary system } \\
\text { (exoskeleton-based); } \\
\text { physical therapy }\end{array}$ & $\begin{array}{l}\text { Commercial system; Cl } \\
\text { studies: } 3 \text { cS (ARMin I) } \\
\text { [91], } 4 \text { cS (ARMin II) [92] }\end{array}$ \\
\hline $\begin{array}{l}\text { ArmeoSpring, Hocoma } \\
\text { AG; based on: T-WREX, } \\
\text { Sanchez [106] }\end{array}$ & $\{7\}$ & $\begin{array}{l}\text { \{Shoulder - FE, } \\
\text { AA, RT, elbow - } \\
\text { FE, forearm - } \\
\text { PS, wrist - FE, } \\
\text { fingers - GR\} }\end{array}$ & $\begin{array}{l}\text { Joint angles, } \\
\text { grasp force }\end{array}$ & None & $\begin{array}{l}\text { Stationary system } \\
\text { (exoskeleton-based); } \\
\text { physical therapy }\end{array}$ & $\begin{array}{l}\text { Commercial system (CE } \\
\text { Mark, FDA clearance); } \\
\text { Cl study: } 10 \text { MS [93]; see } \\
\text { also: T-WREX }\end{array}$ \\
\hline ARMOR, Mayr [177] & 8 & $\begin{array}{l}\text { Shoulder - FE, AA, } \\
\text { RT, elbow - FE, } \\
\text { forearm - PS, } \\
\text { wrist - FE, } \\
\text { thumb - FE, } \\
\text { other fingers } \\
\text { together - FE }\end{array}$ & $\begin{array}{l}\text { Joint angles } \\
\text { of the master } \\
\text { hand }\end{array}$ & Electric motor & $\begin{array}{l}\text { Stationary master- } \\
\text { slave system } \\
\text { (exoskeleton-based); } \\
\text { physical therapy }\end{array}$ & Cll study: 8(8) sS \\
\hline $\begin{array}{l}\text { Gentle/G, Loureiro } \\
{[123]}\end{array}$ & $6\{+3\}$ & $\begin{array}{l}\text { Shoulder* } \\
\text { elbow (3 DOF, } \\
\text { HapticMaster), } \\
\text { \{forearm - PS, } \\
\text { wrist - FE, AA\}, } \\
\text { thumb - FE, } \\
\text { other fingers } \\
\text { together - FE (x2) } \\
\text { (grasp robot) }\end{array}$ & $\begin{array}{l}\text { End-point } \\
\text { torque, position } \\
\text { and velocity } \\
\text { (HapticMaster) } \\
\text { joint angels and } \\
\text { end-point force } \\
\text { (grasp robot) }\end{array}$ & $\begin{array}{l}\text { DC brushed } \\
\text { motors } \\
\text { (HapticMaster and } \\
\text { grasp robot) }\end{array}$ & $\begin{array}{l}\text { Stationary system } \\
\text { (robotic arm + } \\
\text { orthosis); physical } \\
\text { therapy }\end{array}$ & $\begin{array}{l}\text { Cll study: 4(4) sS [94]; } \\
\text { based on Gentle/S }\end{array}$ \\
\hline HEnRiE, Mihelj [124] & $4\{+2\}$ & $\begin{array}{l}\text { Shoulder * elbow } \\
\text { (3 DOF, Haptic- } \\
\text { Master), \{wrist - } \\
\text { FE, AA\}, thumb, } \\
\text { middle and index }\end{array}$ & $\begin{array}{l}\text { End-point } \\
\text { torque, position } \\
\text { and velocity } \\
\text { (HapticMaster) } \\
\text { joint angels and }\end{array}$ & $\begin{array}{l}\text { DC brushed } \\
\text { motors } \\
\text { (HapticMaster) } \\
\text { electric motors } \\
\text { (grasping device) }\end{array}$ & $\begin{array}{l}\text { Stationary system } \\
\text { (robotic arm + } \\
\text { orthosis); physical } \\
\text { therapy }\end{array}$ & $\begin{array}{l}\text { Prototype (with spring } \\
\text { instead of an actuator in } \\
\text { the hand part); CO study: } \\
\text { hs; based on Gentle/s }\end{array}$ \\
\hline
\end{tabular}

middle and index joint angels and finger together - end-point force (grasping device) 
Table 1 Robotic devices for upper limb rehabilitation (Continued)

\begin{tabular}{|c|c|c|c|c|c|c|}
\hline IntelliArm, Ren [116] & $8\{+2\}$ & $\begin{array}{l}\text { Shoulder - FE, AA, } \\
\text { RT, VD, }\{H D(\times 2)\} \text {, } \\
\text { elbow - FE, fore- } \\
\text { arm - PS, wrist } \\
\text { - FE, all fingers } \\
\text { together - GR }\end{array}$ & $\begin{array}{l}\text { Joint angles } \\
\text { and torques }\end{array}$ & Not specified & $\begin{array}{l}\text { Stationary system } \\
\text { (exoskeleton- } \\
\text { based); physical } \\
\text { therapy }\end{array}$ & $\mathrm{Cl}$ study: stroke (np) \\
\hline $\begin{array}{l}\text { MUNDUS, Pedrocchi } \\
\text { [101] }\end{array}$ & {$[3]+\{2\}+1$} & $\begin{array}{l}\text { [Shoulder - FE, } \\
\text { AA, elbow - FE], } \\
\text { optional: forearm - } \\
\text { PS, wrist - FE } \\
\text { (shoulder-elbow- } \\
\text { wrist exoskeleton), } \\
\text { optional: all } \\
\text { fingers together - } \\
\text { GR (hand orthosis) }\end{array}$ & $\begin{array}{l}\text { sEMG, button, } \\
\text { eye-movement } \\
\text { or Bran Computer } \\
\text { Interface; object } \\
\text { labels - radio } \\
\text { frequency } \\
\text { identification }\end{array}$ & $\begin{array}{l}\text { elastic elements } \\
\text { or DC brakes } \\
\text { (shoulder- } \\
\text { elbow-wrist } \\
\text { exoskeleton), FES } \\
\text { (optional), DC } \\
\text { motor (optional } \\
\text { hand orthosis) }\end{array}$ & $\begin{array}{l}\text { Modular } \\
\text { wheelchair- } \\
\text { mounted system } \\
\text { (exoskeleton- } \\
\text { based); } \\
\text { movement } \\
\text { assistance }\end{array}$ & $\mathrm{Cl}$ study: $3 \mathrm{SCl}+2 \mathrm{MS}$ \\
\hline $\begin{array}{l}\text { ReoGo, Motorica } \\
\text { Medical Inc. }\end{array}$ & $2+\{1\}$ & $\begin{array}{l}\text { Shoulder * elbow; } \\
\text { also }\left\{{ }^{*} \text { wrist }\right\} \text { or } \\
\text { \{fingers - FE }\} \text { if } \\
\text { special handle } \\
\text { used }\end{array}$ & $\begin{array}{l}\text { End-point posi- } \\
\text { tion }\end{array}$ & $\begin{array}{l}\text { Electric motors } \\
(x 4)\end{array}$ & $\begin{array}{l}\text { Portable system } \\
\text { (end-effector- } \\
\text { based) with } \\
\text { various handles; } \\
\text { physical therapy }\end{array}$ & $\begin{array}{l}\text { Commercial system; } \\
\text { CIII/CIV study: 60(np) sS } \\
\text { [198], Cl studies: } 14 \text { CS [95], } \\
10 \text { sS [96] }\end{array}$ \\
\hline
\end{tabular}

All the systems in the following table are grouped according to the joint movement they support. For the sake of convenience, we consider the shoulder complex, the forearm and the hand (fingers) as single joints. Thus, we distinguish the following "joints": shoulder, elbow, forearm, wrist and fingers. Devices assisting movements of only one "joint" (starting from shoulder and ending with fingers) are described first followed by devices assisting movement of two, three and four joints (in that order). The end of the table presents systems assisting movement of the whole arm.

For some systems it was difficult to classify them into a particular group. One of such cases includes the end-effector-based systems with a splint. A specific classification to particular group may depend on the joints constrained in particular case by the splint. Furthermore, some devices allow for movements in some joints only in a limited range.

In some cases the same system may appear multiple times in the table on various stages of development. We have accepted such occurrences only if, in our opinion, the difference between two versions of the system justified considering them as two various systems. Otherwise, information included in the table includes only the most recent version of the system available at the time of this publication.

System names are provided in italics. Whenever possible, the first column of the table provides the system name and reference (including the name of the first author) to the publication in which the system is described. We only provide the appropriate reference for systems without a name. The names of commercial systems are followed by their producer names. Appropriate information is provided following a semicolon for commercial systems based on systems being described in scientific publication before commercialization. Except one case, i.e. ArmeoSpring based on T-WREX system, the description of the predecessors is not provided elsewhere in the table because we found no significant differences between the predecessors and their commercial versions.

The last column contains information about the current stage of system development, clinical trials performed using the system and some additional information are provided. If the system has undergone clinical evaluation, information about the category of the trial, number of participants enrolled and their condition, as well as reference to the paper presenting results of the study is also provided. We distinguish four categories of the studies marked as $\mathrm{CO}$, Cl, CII, CIII/CIV. For a description, see Table 7. Categories CII and CIII/CIV provide two numbers of subjects. The first number indicates the total number of participants enrolled in the study. The number in parenthesis indicates number of participants undergoing therapy using the particular system. We made this distinction because there is often a control group undergoing other form of therapy in the CII and CIII/CIV studies. If both numbers are equal, all participants underwent therapy using the specified system but other parameter of the study varied between the groups (e.g. training intensity, device control strategy, or order in which various forms of therapy were applied). No reference after the number and condition of participants indicates that the reference is the same as the one provided in the first column. Information about predecessors or successors is also provided, if available. We use the following symbols and abbreviations:

- for degrees of freedom of the device (DOF) and supported movements (second and third column of the table respectively): [ ] - indicates passive (i.e. exerting only resistive force) and \{\} - indicates not-actuated degrees of freedom or movements, otherwise active.

- for supported movements (third column): (joint name) - indicates that range of movements for that joint is limited to a very small range, AA - adduction/abduction, FE - flexion/extension, GR - grasp and release, PS - pronation/supination, RT - internal/external rotation, HD - horizonatal displacement, VD - vertical displacement (both in the shoulder girdle), MCP - metacarpophalangeal joint, * - indicates that the direction of the movement of the device does not correspond to the direction of any of basic anatomical movements (e.g. pronation/supination, flexion/extension, rotation) but is a combination of many, (x number) - indicates that a few particular movements are possible (e.g. flexion in a few joints of one finger), (in the plane) - indicates that the end effector of the device moves only in a specified plane; for the explanation of anatomical terms of motion see Figure 2.

- for main control inputs and actuators (fourth and fifth column respectively): (commercial system name) - indicates that the particular commercial device (usually robot or haptic interface) is incorporated in the described system and that the particular sensors or actuators are part of that commercial system.

- for main control (forth column): sEMG - surface electromyography.

- for actuators (fifth column): AC - alternating current, DC - direct current, ERF - electrorheological fluid based, FES - functional electrical stimulation, MRF -

magnetorheological fluid based, PAM - pneumatic artificial muscle, SEA - series elastic actuator, (x number) - number of particular actuators being used (provided only if such an information was available).

- for clinical studies (last column): C0, Cl, CII, CIII/CIV - category of the study: 0, I, II and III/IV, respectively (for category descriptions see the subsection Clinical studies of the survey); subject condition: aS - acute stroke, CP - cerebral palsy, CS - chronic stroke, ET - essential tremor, hs - healthy subject(s), MS - multiple sclerosis, SCI - spinal cord injury, sS - subacute stroke, TBI - traumatic brain injury; $\mathrm{np}$ - number of subjects is not provided.

new and improved devices for robotic upper limb rehabilitation. The aim of this paper is to summarize existing technical solutions for physical therapy of the upper limb.

The survey of robotic devices is comprised of advanced technology systems. As defined in this report, the design of advance technology systems includes sensors, actuators, and control units; purely mechanical solutions are excluded from this survey. Although the research team made an effort to identify as many systems as possible, it is reasonable to acknowledge that many systems 
are left unmentioned. Nevertheless, this documentation is intended to be a valuable source of information for engineers, scientists and physiotherapists working on new solutions for physical rehabilitation.

\section{The survey}

\section{Scope of the survey}

At the outset, the research team identified literature associated with the subject matter based on searches in PubMed, the Institute of Electrical and Electronics Engineers (IEEE), Science Direct and Google Scholar databases using various combinations of the following keywords: upper extremity, arm, hand, rehabilitation, therapy, training, movement, motion, assistance, support, robot, robotized, robotic, mechatronic, and motorized. Additionally, referenced literature from the selected publications was included in the survey as well. The information obtained from this literature compendium is supplemented with the data acquired from professional caregivers and manufacturers' catalogs and websites, as well as direct communications with rehabilitation professionals, manufacturers and patients. Over 120 systems are summarized and compared in Table 1; this tabulated summary constitutes the reference for information provided in subsequent sections. As previously mentioned, the scope of this report is generally limited to the devices that support or retrain movement or manipulation abilities of disabled individuals. This survey excludes systems developed for movement assessment, occupational purposes or boosting physical abilities of healthy people. We however considered some academic, not yet specialized systems, supporting upper-extremity movements, especially if they have potential to be used for rehabilitation purposes (e.g. CADEN-7 [97]). This survey also excludes devices that substitute movements of the disabled extremity but do not replace the movement itself (e.g. wheelchair mounted manipulators or autonomous robots). Although these devices improve the patient's quality of life, they differ significantly from systems described in this survey and constitute a separate category of devices. Some companies (e.g. CSMi Computer Sports Medicine, Inc.; Biodex Medical Systems, Inc.; BTE Technologies, Inc.) manufacture sensorized equipment for rehabilitation of various joints and muscles and whose principle of operation often resembles that of exercising devices found at fitness centers. Those devices are used mainly to strengthen muscles and joints and provide some predefined resistance (e.g. isotonic, isometric or isokinetic exercises) or active force (e.g. continuous passive motion exercises). These devices also constitute a different category from the systems included in this survey because their functions are performed along a predefined operation pathway. Although difficult to clearly identify, the aforementioned were also excluded from this review.
Throughout this report, the term "number of degrees of freedom (DOF)" describes the sum of all independent movements (i.e. displacements or rotations) that can be performed in all the joints of the device. The number of DOF is defined in order to determine the exact position and orientation of all segments of the device. Also, some sections in this report are supplemented by an explanation of the most important terminology for readers who are not familiar with the technical vocabulary.

\section{Application field and target group}

A description of the specific field of application for upper limb rehabilitation devices often determines solutions for which the device itself may be applied. Upper-extremity rehabilitation involves actions that stimulate patients' independence and quality of life. Two main application fields of robotic devices stand out: support to perform some ADLs (e.g. by power assistance or tremor suppression) and providing physical training (therapy). Although there is a significant need for powered devices supporting basic ADL at home, there are only a few of such devices proposed so far (see sixth column in Table 1). This is mainly due to technical and economical restrictions. Such devices should significantly improve the lives of their users, otherwise patients become dissatisfied and discontinue their use shortly after. They should be also safe, easily to handle and inexpensive. Portability is also often expected from devices assisting patients to perform basic ADL; in such cases the amount of available energy is limited by the capacity to store energy. Furthermore, if the device is supposed to support movements of multiple joints, the number of needed actuators increases as well as the weight of the device. Therefore, the number of portable actuated devices supporting upper extremity movements is typically low. Instead, purely mechanical solutions are used for that purpose. A few examples of portable powered devices for upper extremity assistance used in daily living are PowerGrip system (Broaden Horizons, Inc., USA) and a system proposed by Hasegawa, et al. [98] (both for grasp assistance), as well as WOTAS orthosis [99] and a system proposed by Loureiro, et al. [100] (both for tremor suppression). However, portability is not always necessary. Often, especially after a stroke or a spinal cord injury, disorders of the upper extremity are accompanied by lower extremity disabilities. These scenarios are typically characterized by immobilized conditions and require a wheelchair. Therefore, many systems assisting upper limb movements are installed close to the patient (e.g. modular wheelchair-mounted system MUNDUS [101]).

Another group of the robotic devices used for rehabilitation purposes, much bigger than the group of devices supporting basic ADLs, constitute devices providing physical therapy. These may be designed for either 
specialized therapeutic institutes or home-based conditions. A vast majority of these devices may be used only at therapeutic institutes since they require supervised assistance from qualified personnel. Their price is often prohibitive for personal use due to their complexity. The patient demand for home-based therapy is expected to increase. Along this context, the concept of the Gloreha system (Idrogenet srl) is provided in two versions: (1) a more complex and more adaptable professional version intended for use at hospitals and rehabilitation centers and (2) a simplified low-cost version intended for patient use at home. However, according to Dijkers, et al. [102], many therapists may stop using devices if set-up takes more than 5 minutes. Thus new developed devices for physical training should be intuitive, easy and fast to set-up and have a reasonable price.

Stroke is the most common cause among diseases and injuries for upper limb movement disorders. It is estimated that by 2030 , stroke will be the fourth leading cause of reduced disability-adjusted life-years (DALY) in western countries. DALY takes into account years of life lost due to premature death as wells as years of life lived in less than full health [103]. Other causes include traumatic brain injury, spinal cord injury and injuries to motoneurons, as well as certain neurological diseases such as multiple sclerosis, cerebral palsy, Guillain-Barre syndrome, essential tremor and Parkinson's disease. Currently proposed robotic systems for upper limb rehabilitation are typically tested on stroke patients. Only a fraction of these systems are investigated on subjects suffering from other diseases (see last column of Table 1).

\section{Type of assistance}

The most important terminology introduced in this section is explained in Table 2. Devices for upper limb rehabilitation may provide different types of motion assistance: active, passive, haptic and coaching. Active devices provide active motion assistance and possess at least one actuator, thus they are able to produce movement of the upper-extremity. Most of the devices discussed in this survey are active (see Table 1). Such assistance of movements is required if patient is too weak to perform specific exercises. However, even with active devices, an exercise is considered passive when a patient's effort is not required. For example, devices providing continuous passive motion exercises are active, but those exercises are categorized as passive because the subject remains inactive while the device actively moves the joint through a controlled range of motion. It is not necessary to apply active assistance to resist patient's movement, to increase patient's force or to ensure the patient is following the desired trajectory. Instead, passive devices may be applied that are equipped with actuators providing resistive force only (i.e. brakes). Such actuators consume less energy and are cheaper than
Table 2 Glossary of terms concerning type of assistance

\begin{tabular}{ll}
\hline Term & Description \\
\hline Active device & $\begin{array}{l}\text { A device able to move limbs. Under such condition, } \\
\text { this device requires active actuators which may } \\
\text { increase the weight. It may also apply to subjects } \\
\text { completely unable to move their limb. }\end{array}$ \\
\hline Passive device & $\begin{array}{l}\text { A device unable to move limbs, but may resist the } \\
\text { movement when exerted in the wrong direction. This } \\
\text { type of device may only be used for rehabilitation of } \\
\text { subjects able to move their limbs. It is usually lighter } \\
\text { than active device since it possesses no actuators } \\
\text { other than brakes. }\end{array}$ \\
\hline Haptic device & $\begin{array}{l}\text { A device that interfaces with the user through the } \\
\text { sense of touch. In most cases it provides some } \\
\text { amount of resistive force, often also some other } \\
\text { sensation (e.g. vibration). It is sometimes also able to } \\
\text { generate specific movements. However, the force it } \\
\text { generates is usually small. Haptic devices are } \\
\text { commonly used in rehabilitation settings with virtual } \\
\text { environments. }\end{array}$ \\
\hline Coaching device & $\begin{array}{l}\text { A device that neither assists nor resists movement. } \\
\text { However, it is able to track the movement and provide } \\
\text { feedback related to the performance of the subject. } \\
\text { As haptic devices, coaching devices are also } \\
\text { commonly used in rehabilitation settings with virtual } \\
\text { environments. }\end{array}$ \\
\hline Passive exercise & $\begin{array}{l}\text { An exercise in which subjects actively move their limb, } \\
\text { although some assistance of the device may be } \\
\text { provided. Such type of the exercise may be performed } \\
\text { using any of the above listed types of devices. }\end{array}$ \\
$\begin{array}{l}\text { An exercise in which the subject remains passive, } \\
\text { while a device moves the limb. This type of exercise } \\
\text { requires an active device. Continuous passive motion } \\
\text { (CPM) training is an example of passive exercise with } \\
\text { active devices. }\end{array}$ \\
\hline
\end{tabular}

the heavier actuators for active assistance. Devices using only resistive actuators include both devices for physical therapy, e.g. MEM-MRB [104] and PLEMO [105], and systems for tremor suppression, e.g. WOTAS [99] orthosis and a system proposed by Loureiro, et al. [100].

Haptic devices constitute another group of systems interacting with the user through the sense of touch. Haptic devices are similarly classified as either active or passive, depending on their type of actuator. In this report, haptic devices are independently categorized because their main function is not to cause or resist movement but rather to provide tactile sensation to the user. Other non-actuated devices for upper limb rehabilitation do not generate any forces but provide different feedback. These systems are labeled coaching devices throughout this report. Because coaching devices are sensorized, they serve as input interface for interaction with therapeutic games in virtual reality (VR) (e.g. T-WREX [106], ArmeoSpring from Hocoma AG) or for telerehabilitation (i.e. remotely supervised therapy). Coaching systems using video-based motion recognition (e.g. Microsoft Kinect) would also belong to this category if it were not for their 
lack of any mechanical part in contact with the patient. Therefore, these systems will not be further discussed in this survey.

Passive and non-actuated systems are less complex, safer and cheaper than their active counterparts. However, they are often modified in the development process with more active characteristics. Still, the main characteristic that identifies a non-actuated or passive device is the lack of the ability to perform movement; they may be an option for continuation of the rehabilitation process, rather than for training of people with significant movement disorders at an early stage of rehabilitation.

\section{Mechanical design}

The most important terminology introduced in this section is explained in Table 3. When comparing the mechanical structure of robotic devices for movement rehabilitation often two categories of devices are considered: end-effector-based and exoskeleton-based. The

Table 3 Glossary of terms concerning mechanical design of robots for rehabilitation

\begin{tabular}{|c|c|}
\hline Term & Description \\
\hline $\begin{array}{l}\text { End-effector based } \\
\text { device }\end{array}$ & $\begin{array}{l}\text { Contacts a subject's limb only at its most distal } \\
\text { part. It simplifies the structure of the device. } \\
\text { However, it may complicate the control of the } \\
\text { limb position in cases with multiple possible } \\
\text { degrees of freedom. }\end{array}$ \\
\hline $\begin{array}{l}\text { Exoskeleton-based } \\
\text { device }\end{array}$ & $\begin{array}{l}\text { A device with a mechanical structure that mirrors } \\
\text { the skeletal structure of the limb, i.e. each segment } \\
\text { of the limb associated with a joint movement is } \\
\text { attached to the corresponding segment of the } \\
\text { device. This design allows independent, } \\
\text { concurrent and precise control of movements in a } \\
\text { few limb joints. It is, however, more complex than } \\
\text { an end-effector based device. Orthoses restricting } \\
\text { or assisting movement in one or more joints may } \\
\text { be also considered exoskeleton-based devices. }\end{array}$ \\
\hline Planar robot & $\begin{array}{l}\text { A device, usually end-effector based, moving in a } \\
\text { specific plane. Design of planar robots, decreases } \\
\text { costs as well as the range of movements that } \\
\text { may be exercised. Although this device performs } \\
\text { movements in a plane, joints of the limb may still } \\
\text { move in a three-dimensional space. }\end{array}$ \\
\hline Back-drivability & $\begin{array}{l}\text { A property of mechanical design indicating that } \\
\text { the patient is able to move the device, even when } \\
\text { the device is in passive state. It increases patient } \\
\text { safety, because it does not constrain limb } \\
\text { movements and keeps patient's limb in a } \\
\text { comfortable position. }\end{array}$ \\
\hline Modularity & $\begin{array}{l}\text { A property of a device indicating that optional } \\
\text { parts may adapt it to a specific condition or simply } \\
\text { to perform additional exercises. }\end{array}$ \\
\hline Reconfigurability & $\begin{array}{l}\text { A property of a device indicating that its } \\
\text { mechanical structure may be modified without } \\
\text { adding additional parts in order to adapt it to the } \\
\text { condition of the subject or to perform other form } \\
\text { of training. }\end{array}$ \\
\hline
\end{tabular}

difference between the two categories is how the movement is transferred from the device to the patient's upper extremity. End-effector-based devices contact the patient's limb only at its most distal part that is attached to patient's upper extremity (i.e. end effector). Movements of the end effector change the position of the upper limb to which it is attached. However, segments of the upper extremity create a mechanical chain. Thus, movements of the end effector also indirectly change the position of other segments of the patient's body as well. Compared to end effectors, exoskeleton-based devices have a mechanical structure that mirrors the skeletal structure of patient's limb. Therefore movement in the particular joint of the device directly produces a movement of the specific joint of the limb.

The advantage of the end-effector-based systems is their simpler structure and thus less complicated control algorithms. However, it is difficult to isolate specific movements of a particular joint because these systems produce complex movements. The manipulator allows up to six unique movements (i.e. 3 rotations and 3 translations). Control of the movements of the patients upper limb is possible only if the sum of possible anatomical movements of patient arm in all assisted joints is limited to six. Increasing the number of defined movements for the same position of the end-point of the manipulator results in redundant configurations of the patient's arm, thus inducing risk of injuries and complicated control algorithms.

The typical end-effector-based systems include serial manipulators (e.g. MIT Manus [107] - Figure 1B, ACRE [108]), parallel (e.g. CRAMER [109] and a system proposed by Takaiwa and Noritsugu [110], both for wrist rehabilitation), and cable-driven robots (e.g. NeReBot [111] - Figure 1C, MACARM [112]). The mechanical structure of HandCARE [113] may be also recognized as the series of end-effector-based cable-driven robots, each of which induce movement of one finger. In this system a clutch system allows independent movement of each finger using only one actuator.

Application of the exoskeleton-based approach allows for independent and concurrent control of particular movement of patient's arm in many joints, even if the overall number of assisted movements is higher than six. However, in order to avoid patient injury, it is necessary to adjust lengths of particular segments of the manipulator to the lengths of the segments of the patient arm. Therefore setting-up such device for a particular patient, especially if the device has many segments, may take a significant amount of time. Furthermore, the position of the center of rotation of many joints of human body, especially of the shoulder complex [114], may change significantly during movement. Special mechanisms are necessary to ensure patient safety and comfort when an 

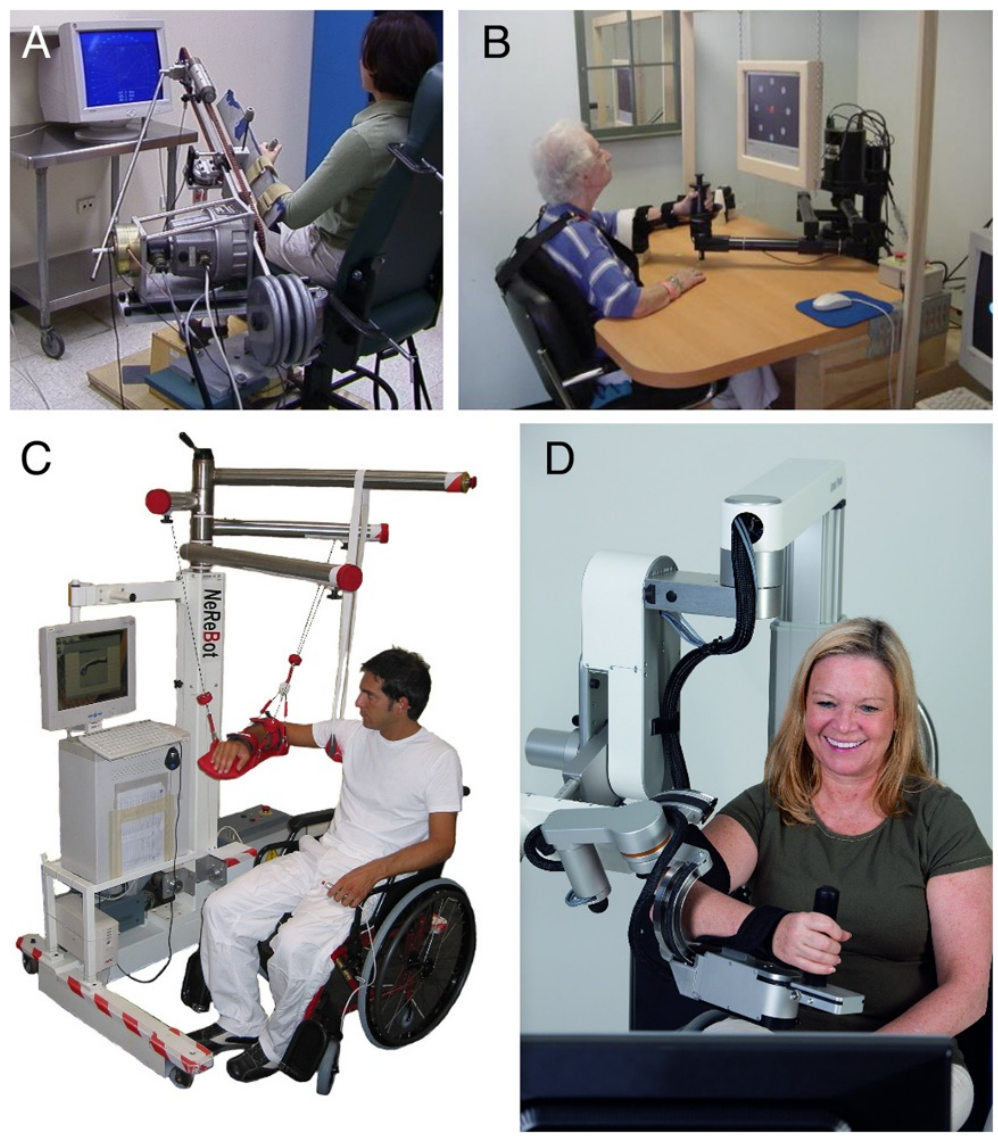

Figure 1 Examples of mechanical structures of robotic devices for upper limb rehabilitation. A: ARM Guide - simple system using linear bearing to modify orientation [136]; B: InMotion ARM - end-effector-based commercial system [133]; C: NeReBot - cable-driven robot, @2007 IEEE. Reprinted, with permission, from [111]; D: ArmeoPower - exoskeleton-based commercial system (courtesy of Hocoma AG).

exoskeleton-based robot assists the movements of these joints [114]. For this reason, the mechanical and control algorithm complexity of such devices is usually significantly higher than of the end-effector-based devices. The complexity escalates as the number of DOF increases.

In case of systems for the rehabilitation of the whole limb the number of DOF reaches nine (ESTEC exoskeleton [115]) or ten (IntelliArm [116]). Some systems for fingers or hand rehabilitation have an even higher number of DOF. Examples include the system proposed by Hasegawa, et al. with eleven DOF [98] and the hand exoskeleton developed at the Technical University (TU) of Berlin with twenty DOF [117]. Even at such a high number of DOF some of these devices still remain wearable (i.e. the user is able to walk within a limited area due to connections to power source and control unit, e.g. ESTEC and hand exoskeleton developed at the TU Berlin) or portable (i.e. the area within which the user may walk is not limited, e.g. the system proposed by Hasegawa).

Apart from purely exoskeleton- or end-effector-based devices, there are many systems combining a few approaches. In the ArmeoSpring system (Hocoma AG) for example, only the distal part - comprising the elbow, forearm and wrist - is designed as an exoskeleton. Therefore the limb posture is statically fully determined (as in exoskeleton-based systems) and the shoulder joint is not constrained, allowing easy individual system adaptation to different patients. A similar concept was applied in Biomimetic Orthosis for the Neurorehabilitation of the Elbow and Shoulder - BONES [118]. In that case, a parallel robot consisting of passive sliding rods pivoting with respect to a fixed frame provides shoulder movements. Such application of sliding rods allows internal/external rotation of the arm without any circular bearing element. The distal part allowing for flexing/extending the elbow resembles the exoskeleton structure. In the MIME-RiceWrist rehabilitation system [119] the end-effector-based MIME [120] system for shoulder and elbow rehabilitation is integrated with the parallel wrist mechanism used in the $M A H I$ exoskeleton (known as RiceWrist [119]).

Another example is the 6 DOF Gentle/S [121] system allowing for relatively large reaching movements (three 
actuated DOF of the end-effector-based commercial haptic interface HapticMaster, Moog in the Netherlands BV [122]) and arbitrary positioning of the hand (connection mechanism with three passive DOF). The Gentle/S system was further supplemented with a three-active-DOF hand exoskeleton to allow grasp and release movements. This new nine DOF system is known as Gentle/G [123].

The HEnRiE [124] is a similar system based on the Gentle/S system. In addition to the three active DOF of HapticMaster, HEnRiE includes a connection mechanism with two passive DOF for positioning of the hand and grasping device (two parallelogram mechanisms allowing parallel opening and closing of fingers attachments) with only one active DOF.

Some systems combine more than one robot at the same time. This approach may be considered as the combination of end-effector-approach, where only the most distal parts of robots are attached to the patient's upper limb, with the exoskeleton-based approach, where movements of few segments are directly controlled at the same time. Use of two robots to control the movements of the limb may allow for mimicking the operations performed by therapist using two hands. Examples of systems using two-robot-concept include REHAROB [125] (using two six-DOF manipulators), iPAM [126] and $U M H$ [127] (both having six DOF in total). Researchers at the University of Twente, in Enschede, Netherlands, made an attempt to use two HapticMaster systems to provide coordinated bilateral arm training, but limitations in hardware and software caused the virtual exercise to behave differently to the real-life [128]. In some cases industrial robots have also been used. The REHAROB uses IRB 140 and IRB $1400 H$ from ABB Ltd., while MIME [120] uses PUMA 560 robot. In general, industrial robots reduce costs; however, such robots have significantly higher impedance than the human upper limb and, according to Krebs, et al. [129], should not be in close physical contact with patients. Therefore most of the robots used for the rehabilitation of the upper limb are designed with a low intrinsic impedance. Some of those devices are also back drivable (e.g. HWARD [130] and RehabExos [131]), meaning that the patient's force is able to cause movement of those devices when they are in passive state. Back-drivability further increases safety of the patient because the device does not constrain patient movements. It also allows for using the device as an assessment tool to measure patient's range of motion.

The majority of the devices presented in Table 1 allow movements in three dimensions; however there are also planar robots, i.e., systems allowing movements only on a specified plane (e.g. MEMOS [132] and PLEMO [105]). Also the MIT Manus system initially allowed movements only on one plane [107]. Subsequently, an anti-gravity module added possibility to perform vertical movements
[133] (Figure 1B). Designing the device as a planar robot reduces the range of movements that can be exercised for particular joint. It also reduces the cost of the device. Furthermore, when the working plane is well selected, the range of training motion may suffice in most of therapeutic scenarios. Some of such planar devices allow changes in the working space between horizontal and vertical (Braccio di Ferro [134]) or even almost freely selecting the working plane (e.g. PLEMO and Hybrid-PLEMO [135]). It further increases the range of possible exercise scenarios while keeping the cost of the device at a minimum.

In the ARM Guide [136] (Figure 1A) and ARC-MIME [137] systems, with which patients practice reaching movements, the working space is limited to linear movements because the forearm typically follows a straight-line trajectory. However the orientation of the slide that assists forearm movements can be adjusted to reach multiple workspace regions and fit different scenarios.

Modularity and reconfigurability are concepts that could reduce therapy costs by adopting therapeutic devices for various disabilities or stages in patient recovery. However there are still only a few systems using these concepts. For example, InMotion ARM robot (the commercial version of MIT Manus, previously called InMotion 2.0) from Interactive Motion Technologies, Inc., may be extended by InMotion WRIST robot (previously InMotion 3.0), developed at MIT [138] as a stand-alone system, and InMotion HAND add-on module (previously InMotion 5.0) for grasp and release training. Another example of modular system is MUNDUS [101], consisting of various modules that may be included depending on the patient condition, starting from muscle weakness to complete lost of residual muscle function. For example as command input residual voluntary muscular activation, head/eye motion, or brain signals may be used. However, this system's complexity might make commercialization of the device very difficult.

A very interesting solution was implemented in the Universal Haptic Drive (UHD) [139]. It has only two DOF and, depending on the chosen configuration, it can train either shoulder and elbow during reaching tasks or forearm (flexion/extension) and wrist. For the latter setting option, it is also possible to select a flexion/extension or pronation/supination training for the wrist. See Figure 2 for an explanation of anatomical terms used for description of upper limb motion.

\section{Actuation and power transmission}

The most important terminology introduced in this section is explained in Table 4. Traditionally, energy to the actuators is provided in three forms: electric current, hydraulic fluid or pneumatic pressure. The selection of the energy source determines the type of actuators used in the system. Most of the devices for upper 


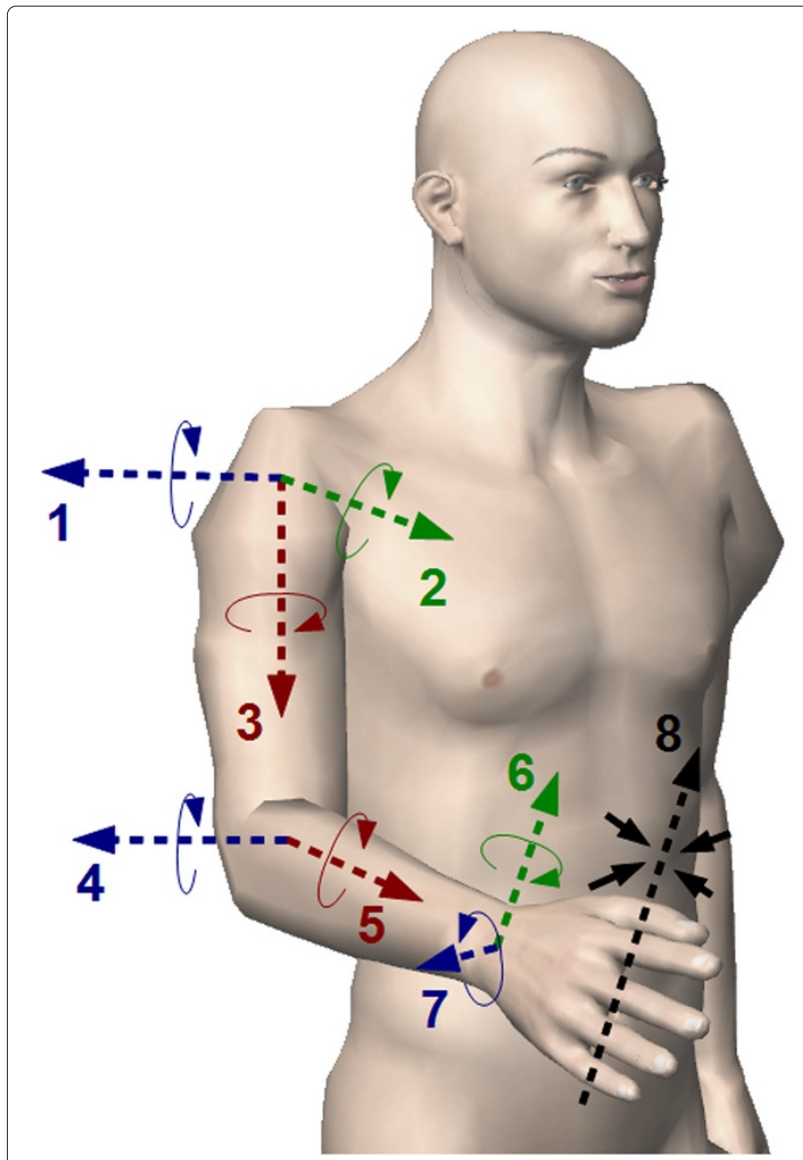

Figure 2 Main movements (degrees of freedom) of the upper extremity. 1: arm flexion/extension; 2: arm adduction/abduction; 3 : arm internal(medial)/external(lateral) rotation; 4: elbow flexion/ extension; 5: forearm pronation/supination; 6: wrist flexion/extension; 7: wrist adduction(ulnar deviation)/abduction(radial deviation); 8 : hand grasp/release.

extremity rehabilitation use electric actuators but there are also other systems with pneumatic and hydraulic actuators. The electric actuators are most common because of their ease to provide and store electrical energy as well as their relatively higher power. Various types and sizes of electrical motors and servomotors are currently commercially available. Some authors (e.g. Caldwell and Tsagarakis [140]) argue that electric actuators are too heavy, compared to their pneumatic counterparts, and their impedance is too high to be used in rehabilitation applications. However, the relatively high powerto-weight ratio of pneumatic actuators is achieved by neglecting the weight of the power source. Adding an elastic element in series with the actuator may also mitigate the high impedance of electric motors. This concept lead to the development of the so called Series Elastic Actuators (SEAs). SEAs decrease inertia and user interface impedance to provide an accurate and stable force
Table 4 Glossary of terms concerning actuation of robots for rehabilitation

\begin{tabular}{|c|c|}
\hline Term & Description \\
\hline Electric actuators & $\begin{array}{l}\text { Actuators powered by electric current. They } \\
\text { are the most common because they easily } \\
\text { provide a relatively high power and are able } \\
\text { to store energy. There is a wide selection } \\
\text { of commercially available electric actuators; } \\
\text { however, some of them are heavy and/or } \\
\text { their impedance is too high for rehabilitation } \\
\text { settings. }\end{array}$ \\
\hline Hydraulic actuators & $\begin{array}{l}\text { Actuators powered by hydraulic pressure } \\
\text { (usually oil). They are able to generate high } \\
\text { forces. Their system is relatively complex } \\
\text { considering the maintenance of pressurized } \\
\text { oil under pressure to prevent leakage. } \\
\text { Commercial hydraulic actuators are also } \\
\text { heavy, therefore, only specially designed } \\
\text { hydraulic actuators are used in rehabilitation } \\
\text { robotics. }\end{array}$ \\
\hline Pneumatic actuators & $\begin{array}{l}\text { Actuators powered by compressed air. They } \\
\text { have lower impedance and weigh less than } \\
\text { electric actuators. Special compressors or } \\
\text { containers with compressed air are required } \\
\text { for power. }\end{array}$ \\
\hline $\begin{array}{l}\text { Pneumatic Artificial } \\
\text { Muscle (PAM, McKibben } \\
\text { type actuator) }\end{array}$ & $\begin{array}{l}\text { A special type of pneumatic actuator with } \\
\text { an internal bladder surrounded by a braided } \\
\text { mesh shell with flexible, but non-extensible } \\
\text { threads. Because of their specific design, an } \\
\text { actuator under pressure shortens, similarly to } \\
\text { the contracting muscle. It is relatively light } \\
\text { and exerts force in a single direction. It is } \\
\text { difficult to control because of its slow and } \\
\text { non-linear dynamic functions. }\end{array}$ \\
\hline $\begin{array}{l}\text { Series Elastic Actuator } \\
\text { (SEA) }\end{array}$ & $\begin{array}{l}\text { A generic name used for a mechanism with } \\
\text { an elastic element placed in series with an } \\
\text { actuator. This solution is relatively often } \\
\text { met in the design of rehabilitation robots. It } \\
\text { decreases the inertia and intrinsic } \\
\text { impedance of the actuator to allow a more } \\
\text { accurate and stable force control and } \\
\text { increase patient safety. }\end{array}$ \\
\hline $\begin{array}{l}\text { Functional Electrical } \\
\text { Stimulation (FES) }\end{array}$ & $\begin{array}{l}\text { It is a technique that uses electrical current to } \\
\text { activate nerves and contract their innervated } \\
\text { muscles. It produces the movement of the } \\
\text { limb using natural actuators of the body. } \\
\text { However, it is difficult to achieve precise and } \\
\text { repeatable movement using this technique } \\
\text { and it may be painful for the patient. }\end{array}$ \\
\hline
\end{tabular}

control [141], thus increasing the safety of the patient. The disadvantage of application with an elastic element is the lower functional bandwidth. Still, the field of rehabilitation does not usually require high bandwidths. SEAs with electric motors are investigated in MARIONET [142] and UHD [139] systems, as well as in systems proposed by Vanderniepen, et al. (referred to as MACCEPA actuators) [143] and Rosati, et al. [144].

A few systems use pneumatic actuators. Pneumatic actuators are lighter and have lower inherent impedance than the electric counterparts. Because such actuators 
require pneumatic pressure, the system is generally either stationary (e.g. Pneu-WREX [145]), its service area is limited (e.g. ASSIST [146]) or the compressor is installed on the patient's wheelchair (e.g. system proposed by Lucas, et al. [147]). Special type of pneumatic actuators, called Pneumatic Artificial Muscles (PAMs), Pneumatic Muscle Actuators or McKibben type actuators are often used in rehabilitation robotics (e.g. Salford Arm Rehabilitation Exoskeleton [148] or system proposed by Kobayashi and Nozaki [149]). Such actuators consist of an internal bladder surrounded by braided mesh shell with flexible, but non-extensible, threads. When the bladder is pressurized, the actuator increases its diameter and shortens according to its volume, thus providing tension at its ends [150]. Due to such physical configuration, PAMs' weight is generally light compared to other actuators, but also have slow and non-linear dynamic response (especially large PAMs), in consequence they are not practical for clinical rehabilitation scenarios [131,151]. In addition, at least two actuators are necessary in order to provide antagonistic movements due to the unidirectional contracting. The ASSIST system has a special type of PAM with rotary pneumatic actuators that allows bending movements [146].

A total of four systems using hydraulic actuators were identified in this survey. All four of them are not standard and use actuators developed specially for that purpose. Reasons to evade industrial hydraulic actuators include weight, impedance, fluid leakages and difficulties to provide fluid. Large, noisy systems are usually necessary for that purpose. Mono-and bi-articular types of Hydraulic Bilateral Servo Actuators (HBSAs) are used in the wheelchair-mounted exoskeleton proposed by Umemura, et al. [152]. Miniaturized and flexible fluidic actuators (FFA) were applied in the elbow orthosis proposed by Pylatiuk, et al. [153]. Hydraulic SEAs are used in two other systems: the Dampace system [154] is equipped with powered hydraulic disk brakes; the Limpact system [155], developed by the same group, uses an active rotational Hydro-Elastic Actuator (rHEA).

In passive systems, it is often desired to modify the amount of resistance during the exercise. This modification increases the resistance when the patient departs from the desired trajectory or to provide haptic feedback for VR interactions. In existing systems, different solutions for provision of adjustable resistive force have been implemented. Powered hydraulic brakes, for example, controlled by electromotors in a SEA are used in Dampace system [154]. Magnetic particle brakes are used in ARM Guide [136] (Figure 1A), in its successor $A R C-M I M E$ [137] to resist other than longitudinal movements of the forearm, and in the device for training of multi-finger twist motion proposed by Scherer, et al. [156]. A few groups have also investigated the application of brakes incorporating magnetorheological (MRF brakes) and electrorheological fluids (ERF brakes). These fluids change their rheological properties (i.e. viscosity) depending on the applied magnetic or electric field, respectively. Thanks to those properties it is possible to achieve brakes with high-performance (with rapid and repeatable brake torque) [105]. MRF brakes are used in MRAGES [157] and MEM-MRB [104] systems. ERF brakes are used in PLEMO [105] and $M R \_C H I R O D$ v.2 [158] systems. The same group that developed the PLEMO also proposed ERF clutches to control the force provided by an electric motor in active systems. Such an actuation system was implemented in EMUL [159], Robotherapist [160] and Hybrid-PLEMO [135] devices.

The natural actuators of body muscles can be used instead of external actuators. For this purpose, an electrical stimulation of the muscles leading to their contraction can be applied. This specific electrical stimulation is known as Functional Electrical Stimulation (FES). FES significantly reduces the weight of the device. From a therapeutic point of view, FES allows patients to exercise muscles, improving muscle bulk and strength and preventing muscular atrophy [161]. It has been also shown that FES, complemented by conventional physiotherapy, may enhance the rehabilitation outcome [162]. However, FES may cause strong involuntary muscle contractions and can be painful for patient. Furthermore, it is difficult to control movements using FES because of the non-linear force characteristic of contracting muscles, muscles fatigue and dependency of the achieved contraction on the quality of the contact between stimulating electrodes and the body tissue. There are two commercial systems using FES for upper limb rehabilitation: Ness H200 (Bioness, Inc., US) and NeuroMove (Zynex Medical, Inc., US). Two other systems combining FES with assistive force were proposed by Freeman, et al. [163] and Li, et al. [164].

When selecting actuators, it is also important to consider their location, especially with exoskeleton-based mechanical structures. The actuators can be placed distally, close to the joints on which they actuate (e.g. ArmeoPower system, Figure 1D). This specification simplifies the power transmission by using direct drives. However, it increases the weight of the distal part of the device and inertia and makes it more difficult to control the system. On the other hand, locating the actuators in the proximal part of the device, often in the part that remains constrained, reduces the weight and inertia of the distal part. However, a power transmission mechanism complicates the mechanical structure and may lead to difficulties with control due to friction. For example, the same group who developed InMotion HAND 
system proposed an earlier prototype of the hand module with eight active DOF and cable-driven mechanism for power transmission. The friction in that mechanism and its level of complexity was too high for clinical scenarios [165]. Nevertheless, there are systems, in which power transmission using cables and gear drives was successfully applied, like for example $C A D E N-7$ [97] and SUEFUL-7 [166].

\section{Control signals}

The most important terminology introduced in this section is explained in Table 5. Various signals may be used as control input of the device. Switches are often used to simplify design. Examples include the PowerGrip system from Broaden Horizons, Inc., hand held triggers (e.g. FES based system for grasp assistance proposed by Nathan, et al. [167]) and a joystick (e.g.MULOS [168]). Most of the systems having more complex control strategies use either kinematic, dynamic or a mix of both input signals (see Table 1 for a comparison). The type of the signal used as control input is partially determined by the low-level control strategy and vice-versa. In some cases, signals provided by actuator encoders (concerning position or torque) may be directly used for control purposes. However, usually torque measured by the encoder is a sum of the torque exerted by the user on device and internal torques in the device. Therefore, for better control of forces between patient and device, it is useful to apply additional sensors that will measure those forces directly.

Some systems use surface electromyography (sEMG) as an input signal, which provides information about intention of the person to perform particular movement. Therefore it is possible to detect and support it. Most of such systems support elbow movements, as sEMG signals from muscles controlling this joints (i.e. biceps brachii or triceps brachii) are relatively easily measured. Among proposed solutions are both stationary (e.g. systems proposed

Table 5 Glossary of terms concerning input control signals of robots for rehabilitation

\begin{tabular}{ll}
\hline Term & Description \\
\hline Dynamic signals & $\begin{array}{l}\text { Signals related to the torque or force exerted } \\
\text { by the subject on various joints of the device } \\
\text { (exoskeleton-based device) or at its end effector } \\
\text { (end-effector-based device). }\end{array}$ \\
\hline Kinematic signals & $\begin{array}{l}\text { Signals related to positions, orientations, velocities } \\
\text { and accelerations of particular segments or joints } \\
\text { of the device or of the limb. }\end{array}$ \\
\hline Trigger signal & $\begin{array}{l}\text { A signal initiating a specific action. In simple cases, } \\
\text { a switch or a button triggers the signal. In more } \\
\text { complex cases, a threshold value of some signal is } \\
\text { specified to trigger the action (e.g. a sEMG value } \\
\text { corresponding to a level of muscle contraction). }\end{array}$ \\
\hline
\end{tabular}

by Rosen, et al. [169] and Kiguchi, et al. [170]) and portable systems (e.g. systems proposed by Ögce and Özyalçin [171] and Pylatiuk, et al. [153]). So far the most successful of those systems is the one DOF portable orthosis developed at the Massachusetts Institute of Technology (MIT), Cambridge, US [172]. The system successfully sustained clinical trials, received FDA approval and was commercialized as Myomo e100 system (Myomo, Inc.) [173]. Examples of sEMG-controlled systems supporting movements of other joints include those proposed by Kiguchi, et al. [114] for shoulder rehabilitation, W-EXOS [174] for forearm and wrist rehabilitation, PolyJbot [175] for wrist rehabilitation, SUEFUL-7 [166] exoskeleton for whole limb (excluding fingers) movement assistance, TU Berlin Hand Exoskeleton [117] for fingers rehabilitation, as well as 11-DOF portable orthosis for grasp assistance proposed by Hasegawa, et al. [98]. The sEMG signals from the contralateral healthy limb have been also used to control movements of the affected one (see system proposed by $\mathrm{Li}$, et al. [176]). The concept of using movements of the not affected limb to control motion of the affected one has been also implemented in Bi-Manu-Track system (Reha-Stim, Germany), ARMOR exoskeleton [177] and device proposed by Kawasaki, et al. [178]. Using the other limb to control the affected one is especially useful during rehabilitation after stroke. In cases of hemiparesis (or hemiplegia), often only one side of the body is affected.

In some systems also contact-less movement detection methods have been used. For example, Ding, et al. [179] proposed a system to assist the load of arbitrary selected muscles using motion capture systems in order to calculate the actual muscle force.

\section{Feedback to the user}

Different types of feedback may be provided to the user, among them: visual, tactile, audio and in the form of electrical stimulation. Some systems, for example those proposed by Lam, et al. [180] and Nathan, et al. [167], use vibrational stimulation of the muscle tendons to support their contraction. It was also suggested that providing tactile feedback to flexor and extensor surfaces of the skin at the appropriate location could produce more naturalistic movements and improve clinical outcomes [3]. Some other systems combine other types of the feedback, for example system proposed by Casellato, et al. [181] combines visual and haptic feedback to improve motor performance of children with dystonia.

A significant number of systems provide training in virtual reality (VR) scenarios. VR provides a much more interesting training surrounding to the patient, compared to the typically available conditions presented in therapeutic units. Furthermore, it allows for fast modification of 
training scenarios, increasing patient attention and motivation to perform the exercise. Therefore it may also improve positive outcome of the therapy. It also adapts the system for various patients in a very short time frame and restarts the task if the object was dropped or misplaced. Haptic devices are especially well suited for provision of therapy in VR because they provide an impression of manipulating the virtual objects. Some groups developed own versions of haptic systems. For example Takahashi, et al. [182] proposed haptic device for arm rehabilitation, which can apply multiple types of force including resistance, assistance, elasticity, viscosity and friction. Other examples are: a two DOF Haptic Interface for Finger Exercise (HAFI), which provides rehabilitation of only one finger at a time [183]; a force reflecting glove, named MRAGES, using magnetorheological fluid [157]; MR_CHIROD v.2, a one DOF grasp exercise device for functional magnetic resonance imaging [158] and force-feedback glove Rutgers Master II-ND [184], developed at the Rutgers University (Piscataway, US) and used in hand therapy scenarios (e.g. [185-187]).

Many groups have investigated application of a few of commercial haptic devices for rehabilitation of upper extremity. Among such haptic interfaces are:

- HapticMaster incorporated for example in Gentle/S [121] (for other examples see Table 1),

- in-parallel robots Phantom Omni and Premium (Geomagic, Inc., US) - used e.g. in experiments performed by Casellato, et al. [181], Brewer, et al. [188], and Xydas and Louca [189],

- parallel robot Falcon (Novint Technology, Inc., US) used in My Scrivener system for hand writing training (Obslap Research LLC, US) [190],

- force-feedback glove CyberGrasp (CyberGlove Systems LLC,US) - used among others in therapeutic scenarios investigated by Adamovich, et al. [191,192].

Because the entertainment industry have recently introduced many new devices to capture motion of the healthy people for interaction with VR-based games, it may be expected that soon some of those devices will be also adapted for rehabilitation purposes, providing so called "serious games".

\section{Control strategy}

The most important terminology introduced in this section is explained in Table 6. Following the example of Marchal-Crespo and Reinkensmeyer [193] we will consider "high-" and "low-level" control strategies used by rehabilitation robots. "High-level" control algorithms are explicitly designed to provoke motor plasticity whereas "low-level" strategies control the force, position, impedance or admittance factors of the "high-level" control strategies.
Table 6 Glossary of terms concerning control strategy of robots for rehabilitation

\begin{tabular}{ll}
\hline Term & Description \\
strategy & $\begin{array}{l}\text { A control strategy with control algorithms } \\
\text { explicitly designed to induce motor plasticity. }\end{array}$ \\
\hline Assistive control & $\begin{array}{l}\text { A "high-level" control strategy in which a device } \\
\text { provides the physical assistance to aid the patient } \\
\text { in accomplishing an intended movement. }\end{array}$ \\
\hline $\begin{array}{l}\text { Challenge-based } \\
\text { control }\end{array}$ & $\begin{array}{l}\text { A "high-level" control strategy in which a device } \\
\text { challenges the patient to accomplish an intended } \\
\text { movement. }\end{array}$ \\
\hline Haptic stimulation & $\begin{array}{l}\text { A "high-level" control strategy in which a robotic } \\
\text { device is used as a haptic interface to perform } \\
\text { activities in virtual reality environment. }\end{array}$ \\
\hline Couching control & $\begin{array}{l}\text { A "high-level" control strategy in which a robotic } \\
\text { device neither physically assists nor resists the } \\
\text { movement of the subject. It only quantifies and } \\
\text { provides feedback (visual, acoustic or other) } \\
\text { concerning the performance of the subject during } \\
\text { exercise. }\end{array}$ \\
\hline $\begin{array}{l}\text { A control strategy considered in the implementa- } \\
\text { tion of the "high-level" control strategy in a device } \\
\text { by appropriate control of the force, position, } \\
\text { impedance or admittance. }\end{array}$ \\
\hline strategy
\end{tabular}

This terminology is mostly based on the one proposed by Marchal-Crespo and Reinkensmeyer [193].

\section{"High-level" control strategies}

There is a myriad of "high-level" control strategies for robotic movement training. This section briefly summarizes the classification of those strategies presented by Marchal-Crespo and Reinkensmeyer [193]. They identify four categorizes of control strategies: (a) assistive control, (b) challenge-based control, (c) haptic stimulation, and (d) non-contacting coaching. Although some systems may fall into a few of these categorizes, this classification well illustrates main notions in the "high-level" control of robotic devices for upper limb rehabilitation. Those control strategies in most cases correspond also to active, passive, haptic and coaching types of motion assistance described before.

The assistive control strategy makes tasks safer and easier to accomplish, allowing more repetitions. There are four types of assistive strategies: impedance-based, counterbalance-based, EMG-based and performancebased adaptive control. In the impedance-based strategy, the patient follows a particular trajectory. The device does not intervene as long as the patient follows this trajectory. However, as the patient leaves the trajectory, the device produces a restoring force that 
increases along with the deviation from the desired trajectory. Often some margin of deviation from allowed trajectory is accepted before restoring force is provided. Counterbalance-based strategies provide a partial, passive or active weight counterbalance to a limb, those making the exercises easier, as the amount of force needed to move the limb against the gravity may be significantly reduced. EMG-based approach uses the patient's own sEMG signals to either trigger or proportionally control the assistance. Both of those approaches encourage patients' effort. However, the triggered method is more susceptible to slacking, as the patient may learn to provide only the amount of force needed to trigger the assistance. Finally, the performancebased adaptive control strategies monitor the performance of the patient and adapt some aspects of the assistance (e.g. force, stiffness, time, path) according to the current performance of the patient, as well as performance during particular number of preceding task repetitions.

Challenge-based control strategies fall into three groups: resistive, amplifying error and constraint-induced. The resistive strategies resist the desired movements, those increasing the effort and attention of the patient. The error amplifying strategies are based on the theory that faster improvements are achieved when error is increased [194]. Therefore they track the deviations from the desired trajectories and either increase the observed kinematic error or amplify its visual representation on the screen. The constraint-induced robotic rehabilitation strategy, similarly to conventional constraint-induced therapy, promotes the use of the affected limb by constraining the not affected one.

Haptic stimulation strategies make use of haptic devices described above, providing tactile sensation for interactions with virtual reality objects. These strategies support training of basic ADLs in safe conditions and without long set-up. They provide alternate tasks in various environments, attracting attention and providing conditions for implicit learning.

Non-contacting coaching strategy is applied in systems that do not contact participants but rather monitor their activity and provide instructions to the patient. Instructions indicate how to perform particular activities or what should be improved. Since such devices do not contact the patient, they are not applicable for systems described herein. However, this category may be extended to include also some sensorized, but not-actuated exoskeletons, such as the gravity balancing orthosis T-WREX [106].

\section{"Low-level" control strategies}

Different "low-level" control strategies are combined to develop "high-level" rehabilitation strategies. Many "lowlevel" control strategies may be proposed during following stages of development of a robotic rehabilitation device. This report provides a short description of basic approaches and does not include a comprehensive comparison of "low-level" control strategies. General books on control engineering provide a more detailed description, in addition to articles referenced in Table 1.

As the rehabilitation robots interact with human body, it is necessary to consider the manipulator and patient as a coupled mechanical system. The application of force or position control is not enough to ensure appropriate and safe dynamic interaction between human and robot [195]. Other control strategies, such as impedance or admittance control are implemented in most of the robots for upper limb rehabilitation. In the impedance control approach the motion of the limb is measured and the robot provides the corresponding force feedback, whereas in the admittance control approach the force exerted by the user is measured, and the device generates the corresponding displacement. The advantages and disadvantages of the impedance and admittance control systems are complementary [196]. In general, robots with impedance control have stable interaction but poor accuracy in free-space due to friction. This low accuracy can be improved using inner loop torque sensors and low-friction joints or direct drives. Admittance control in contrast compensates the mass and friction of the device and provides higher accuracy in non-contact tasks, but can be unstable during dynamic interactions. This problem is eliminated using SEAs. Devices using admittance control require also high transmission ratios (e.g. harmonic drives) for precise motion control [196]. In some cases both of theses approaches may be combined together. Impedance control strategy has been implemented for example in MIT Manus [107] (Figure 1B) and L-Exos exoskeleton [197], admittance control is found in MEMOS [132] and $P P A M$ [126].

\section{Clinical evidence Clinical studies}

As previously discussed, there has been a significant effort during last two decades to improve the design and control strategy of robotic rehabilitation devices. Yet, less has been done to prove the efficacy of such systems in rehabilitation settings. Although the results of clinical evaluation of therapy applying robots are still sparse, the problem is slowly being recognized. The focus in rehabilitation robotics is starting to move from technical laboratories to clinics. References to clinical trials in which robotic rehabilitation devices have been used are provided in the last 
column of Table 1. The classification of clinical trials used in this review is summarized in Table 7.

From the developer and manufacturer's point of view, there may be at least three objectives in performing clinical trials. The first one is to address regulatory requirements. The devices described in this review are considered medical devices in most countries and as such the studies proving device efficacy and safety may be required before they are authorized for distribution. Although in some cases the exemption from providing the clinical data may be granted, e.g. if the device is recognized as low risk (Class 1 device in the European Union and the USA) or if equivalent device has been already approved for commercialization, the clinical data may be required by health insurance authorities in order to provide reimbursement. In this case the objective of the trial is to obtain a proof of clinical or financial benefit of the use of the device as compared to the existing modes of therapy. The third objective of clinical trials is to provide the professional community with the clinical evidence of device's efficacy. Although, the three objectives may seem to be similar, the requirements are not the same, therefore when designing a clinical trial it should be considered if the obtained results will allow to satisfy requirements of those three objectives. For the study design requirements to satisfy the marketing and reimbursement objectives, we refer the readers to the legal regulations in the country of interest. Whereas, for a review on the process to design a clinical train with sound scientific results we refer to Lo [198].

From the clinical point of view, the objective of the clinical study may be different than to validate a particular device. For therapists the robotic device is a tool

Table 7 The classification of clinical trials of rehabilitation robots used in this review

\begin{tabular}{ll}
\hline Term & Description \\
\hline Category 0 & $\begin{array}{l}\text { Initial feasibility studies: Trials performed with low } \\
\text { number of healthy volunteers, often using the prototype } \\
\text { of a device, in order to evaluate its safety and clinical } \\
\text { feasibility. }\end{array}$ \\
\hline Category I & $\begin{array}{l}\text { Pilot Consideration-of-Concept studies: Clinical trials } \\
\text { aimed at testing device safety, clinical feasibility and } \\
\text { potential benefit. They are performed in a small } \\
\text { population of subjects suffering from the target disease. } \\
\text { There is either no control group in the trial, or healthy } \\
\text { subjects are used as control. }\end{array}$ \\
\hline Category II & $\begin{array}{l}\text { Development-of-Concept studies: Clinical studies } \\
\text { aiming at verification of device efficacy. Include a } \\
\text { standardized description of the intervention, a control } \\
\text { group, randomization and blinded outcome assessment. }\end{array}$ \\
\hline Category III/IV & $\begin{array}{l}\text { Demonstration-of-Concept-Studies/Proof-of-Concept } \\
\text { studies: Further evaluation of the device efficacy. Similar } \\
\text { to category II, however, usually these are multicentered } \\
\text { studies with high number of participants. }\end{array}$
\end{tabular}

This classification is based on guidelines provided by Lo [198] and supplemented by Category 0 . that provides a therapy protocol rather than an end product [198], thus they are rather interested in responses to questions concerning optimal training intensity, disorders for which such form of training may be beneficial, and whether robotic therapy should substitute or complement other forms of therapy.

This survey includes a search into the US Clinical Trials database (http://clinicaltrials.gov/) from October 2013 using a combination of keywords: robotic, robot, therapy and rehabilitation. This is an web-based database existing since 1997 and maintained by the US National Library of Medicine at the National Institutes of Health. Under the American Food and Drug Administration Amendments Act (FDAAA) of 2007 all the applicable clinical trials (what concerns category II and III/IV studies in our survey) performed in the USA and starting after 2007 have to be registered in this database. However, it includes also some category I studies and many other studies performed in other countries. Results of this search identified 197 clinical trials out of which 62 are relevant to this survey. The selected trials are divided into two categories. The main objective of the first category is to proof the efficacy or safety of the device, therefore there was either no control group or a control group was undergoing the standard form of the therapy. The main objective of the second category is to determine a more efficient form of the therapy. In the latter category, the participants were assigned to groups undergoing similar forms of therapy, but at different intensities, using various devices or undergoing various forms of therapy in different order. A total of 31 studies aim at device safety or efficacy validation while 27 address better forms of therapy. A total of four trials were excluded. The objective of these trials was to validate other forms of therapy; devices described in this review have only been used as a reference. As indicated in Figure 3, the number of participants enrolled in studies for therapy improvement significantly increased during last three years compared to the number of participants in the device safety/efficacy validation studies. This suggests that the objective of the studies changes from validating forms of therapy to finding optimal applications methods. This survey identified a total of 21 devices out of the 58 clinical studies. However, it was not possible to determine the robotic device in 11 studies. Surprisingly, almost only stroke survivors (54 studies) were enrolled. In the remaining four studies subjects with cerebral palsy, spinal cord injury, traumatic brain injury and rotator cuff tear were involved.

\section{Outcomes of clinical studies}

Many questions concerning effective robotic upper-limb rehabilitation still remain unanswered. One of the most important reasons is that the most effective interventions to optimize neural plasticity are still not clear and it is not 


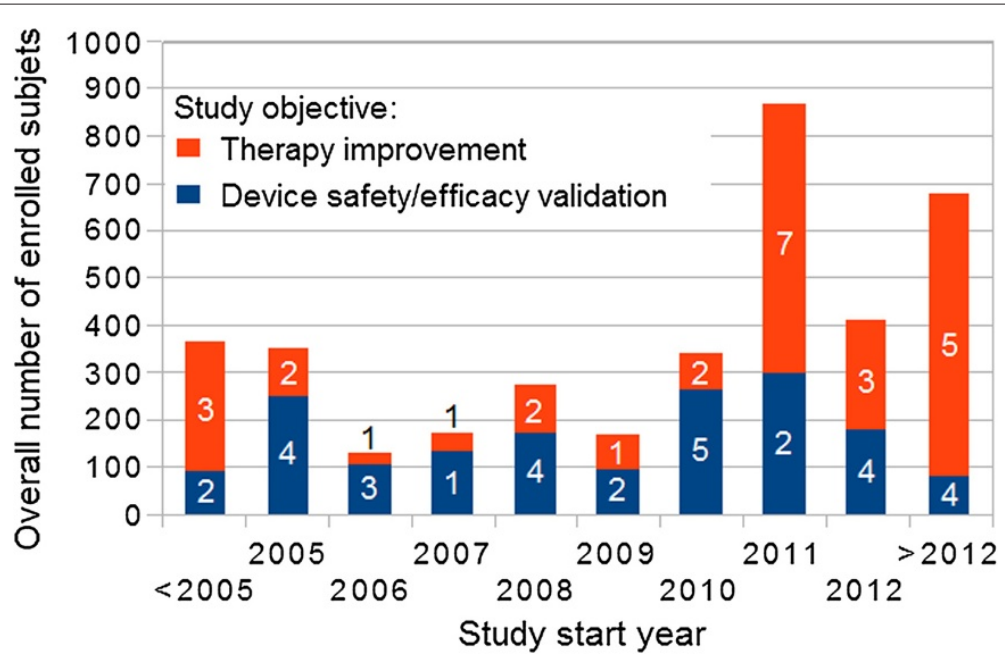

Figure 3 Number of clinical studies and enrolled subjects depending on study objective and start year. Results based on a search in the clinicaltrials.gov database in October 2013. Numbers on and above the bars indicate the number of studies in each category starting in the particular year.

possible to implement them in rehabilitation robotics [7]. The other is that the results of the clinical controlled trials remains limited and those already available are difficult to compare with each other $[7,193]$. It is also questionable which measures should be used to evaluate the effects of therapy and which outcome should be compared: shortterm or long-term. Scales based on evaluation of abilities influencing the quality of life are often not objective enough, since they rely on therapist expertise.

Although it is not possible to indicate the best control strategy for the rehabilitation, there is already some evidence showing that some strategies are producing better outcomes, whereas some can even decrease recovery time compared to possible non-robotic strategies [193].

Most accepted theories about robotic rehabilitation are clear: The goal of the rehabilitation training is not only to maximize the number of repetition but to maximize the patients attention and effort as well [3]. The monotonous exercises provide worse retention of a skill compared with alternate training [7]. Adaptive therapy and assistance as needed provide better results as fixed pattern therapy [193]. Robotic therapy can possibly decrease recovery if it encourages slacking since the patient may decrease effort and attention due to the use of adaptive algorithm [193]. Because learning is error based, faster improvement may be achieved when error is increased [194]. Implicit learning, allowing patients to learn skills without awareness, may result in greater learning effect [7]. Many functional gains are more dependent on wrist and hand movements than on the mobility of shoulder and elbow [7]. It is not the maximal voluntary contraction (strength of the muscles) but appropriately timed activity of agonist and antagonist (coordination of the movements) that significantly improve the rehabilitation [3].

As previously stated, the objective of this report is not to review the results of clinical studies performed so far. A detailed review of clinical studies is referenced in other publications [7,198-202]. The most important results are still worth mentioning. Systematic review and meta-analysis of the trials performed in stroke patients suggest that robotic training improves motor impairment and strength but do not improve ability to perform ADLs $[199,200]$. The results of the first large randomized multicenter study in which training with MIT-Manus robotic system have been compared with intensive therapistprovided therapy and usual care have revealed that there is no significant difference in the outcomes of the two intensive forms of the therapy [203]. Thus the most important advantage of robotic systems is their ability to provide intensive repetitive training without overburdening therapists [204]. Another advantage is the ability to provide more motivating training context, by means of a computer gaming environment with quantitative feedback to motivate practice [205]. Concerning cost-effectiveness of robotic rehabilitation, the results of the previously mentioned multicenter trial have shown that when the total cost of the therapy is compared, i.e. the cost of the therapy plus the cost of all the other health care use, the costs of the two forms of the intensive therapy (i.e. robot-assisted and therapist-provided) are similar [203]. However, the cost of technology is expected to decrease, as opposed to the cost of human labor. Therefore cost-effective advantage toward robot-therapy may be expected [198]. 


\section{Conclusions}

Due to population changes, shortage of professional therapists, and the increasing scientific and technical potential, many research groups have proposed devices with the potential to facilitate the rehabilitation process. Many devices for upper limb rehabilitation have already been proposed. A vast majority of these proposed devices are technically advanced and are designed for clinical settings. However, there is still significant need to improve efficiency and reduce cost of home-based devices for therapy and ADLs assistance. The effectiveness of robotic over conventional therapy is arguable and the best therapy strategy is still not clear. The situation may change soon, because more and more devices are being commercialized and more scientific results will be available. It may encourage next groups to propose their own solutions. Developing new devices and improving those already in the market will be easier, when taking advantage from the already existing solutions. We hope that this survey will help to navigate between those solutions and select best of them, thus facilitating development of new and better systems for robotic upper limb rehabilitation. We also hope that it will be a valuable source of information for all the professionals looking for a comprehensive reference.

\section{Competing interests}

The authors declare that they have no competing interests.

\begin{abstract}
Authors' contributions
PM performed systematical review of papers and drafted the manuscript. JE, $\mathrm{KGH}$, and $\mathrm{AJ}$ contributed to the review of various systems and revised the manuscript. SL contributed to the concept, paper structure and revised the manuscript. All authors read and approved the manuscript.
\end{abstract}

\section{Acknowledgements}

We would like to thank Mrs. Christine Azevedo-Coste, Ph.D. for her critical reading and revising the manuscript and Mr. Gabriel David Nevarez, P.E. for proofreading the English.

The work of PM has been supported by the European Union in the framework of European Social Fund through the Warsaw University of Technology Development Program. He would also like to thank the Center for Advanced Studies of the Warsaw University of Technology for the support that he received during preparation of this work and the Chair of Medical Engineering (mediTEC), Helmholtz-Institute for Biomedical Engineering, RWTH Aachen University for the possibility of performing the majority of the work on this manuscript during scientific stay in that institution.

\footnotetext{
Author details

${ }_{1}^{1}$ DEMAR - LIRMM, INRIA, University of Montpellier 2, CNRS, Montpellier, 161 rue Ada, 34095 Montpellier, France. ${ }^{2}$ Institute of Metrology and Biomedical Engineering, Warsaw University of Technology, ul. Św. A. Boboli 8, 02-525 Warszawa, Poland. ${ }^{3} \mathrm{Helm}$ holtz-Institute for Biomedical Engineering, RWTH Aachen University, Pauwelsstraße 20, 52074 Aachen, Germany. ${ }^{4}$ Chair of Medical Engineering (mediTEC), Helmholtz-Institute for Biomedical Engineering, RWTH Aachen University, Pauwelsstraße 20, 52074 Aachen, Germany. ${ }^{5}$ Philips Chair of Medical Information Technology (MedIT), Helmholtz-Institute for Biomedical Engineering, RWTH Aachen University, Pauwelsstraße 20, 52074 Aachen, Germany.
}

\section{References}

1. Platz T: Evidenzbasierte Armrehabilitation: Eine systematische Literaturübersicht [Evidence-based arm rehabilitation-a systematic review of the literature]. Nervenarzt 2003, 74(10):841-849. [http://dx.doi.org/10.1007/s00115-003-1549-7]

2. Feys H, Weerdt WD, Verbeke G, Steck GC, Capiau C, Kiekens C, Dejaeger E, Hoydonck GV, Vermeersch G, Cras P: Early and repetitive stimulation of the arm can substantially improve the long-term outcome after stroke: a 5-year follow-up study of a randomized trial. Stroke 2004, 35(4):924-929. [http://dx.doi.org/10.1161/01.STR.0000121645.44752.f7]

3. Patton J, Small SL, Rymer WZ: Functional restoration for the stroke survivor: informing the efforts of engineers. Top Stroke Rehabil 2008, 15(6):521-541. [http://dx.doi.org/10.1310/tsr1506-521]

4. Richards $L$, Hanson C, Wellborn M, Sethi A: Driving motor recovery after stroke. Top Stroke Rehabil 2008, 15(5):397-411.

[http://dx.doi.org/10.1310/tsr1505-397]

5. WHO: The global burden of disease: 2004 Update. World Health Organization (WHO) 2008. [http://www.who.int/healthinfo/ global_burden_disease/GBD_report_2004update_full.pdf]

6. Riener R, Nef T, Colombo G: Robot-aided neurorehabilitation of the upper extremities. Med Biol Eng Comput 2005, 43:2-10.

7. Brewer BR, McDowell SK, Worthen-Chaudhari LC: Poststroke upper extremity rehabilitation: a review of robotic systems and clinical results. Top Stroke Rehabil 2007, 14(6):22-44. [http://dx.doi.org/10.1310/tsr1406-22]

8. Gopura RARC, Kiguchi K: Mechanical designs of active upper-limb exoskeleton robots: State-of-the-art and design difficulties. In Proc. IEEE International Conference on Rehabilitation Robotics ICORR. Kyoto, Japan; 2009:178-187.

9. Cheng HS, Ju MS, Lin CCK: Improving elbow torque output of stroke patients with assistive torque controlled by EMG signals. J Biomech Eng 2003, 125(6):881-886.

10. Cozens JA: Robotic assistance of an active upper limb exercise in neurologically impaired patients. Rehabil Eng, IEEE Trans 1999, 7(2):254-256.

11. Mavroidis C, Nikitczuk J, Weinberg B, Danaher G, Jensen K, Pelletier P, Prugnarola J, Stuart R, Arango R, Leahey M, Pavone R, Provo A, Yasevac D: Smart portable rehabilitation devices. J Neuroeng Rehabil 2005, 2:18. [http://dx.doi.org/10.1186/1743-0003-2-18]

12. Song $\mathrm{R}$, yu Tong $\mathrm{K}$, $\mathrm{Hu} \mathrm{X}$ : Assistive control system using continuous myoelectric signal in robot-aided arm training for patients after stroke. IEEE Trans Neural Syst Rehabil Eng 2008, 16(4):371-379. [http://dx. doi.org/10.1109/TNSRE.2008.926707]

13. Hu X, Tong KY, Song R, Tsang VS, Leung PO, Li L: Variation of muscle coactivation patterns in chronic stroke during robot-assisted elbow training. Arch Phys Med Rehabil 2007, 88(8):1022-1029. [http://dx.doi.org/10.1016/j.apmr.2007.05.006]

14. Song R, Tong KY, Hu XL, Tsang SF, Li L: The therapeutic effects of myoelectrically controlled robotic system for persons after stroke-a pilot study. Conf Proc IEEE Eng Med Bio/ Soc 2006, 1:4945-4948. [http://dx.doi.org/10.1109/IEMBS.2006.260186]

15. Kung PC, Ju MS, Lin CCK: Design of a forearm rehabilitation robot. In Proc. IEEE 10th International Conference on Rehabilitation Robotics ICORR. Noordwijk, Netherlands; 2007:228-233.

16. Kung PC, Lin CCK, Ju MS, Chen SM: Time course of abnormal synergies of stroke patients treated and assessed by a neuro-rehabilitation robot. In Proc. IEEE International Conference on Rehabilitation Robotics ICORR. Kyoto, Japan; 2009:12-17.

17. Colombo R, Pisano F, Mazzone A, Delconte C, Micera S, Carrozza MC, Dario P, Minuco G: Design strategies to improve patient motivation during robot-aided rehabilitation. J Neuroeng Rehabil 2007, 4:3. [http://dx.doi.org/10.1186/1743-0003-4-3]

18. Hu XL, Tong KY, Song R, Zheng XJ, Lui KH, Leung WWF, Ng S, Au-Yeung SSY: Quantitative evaluation of motor functional recovery process in chronic stroke patients during robot-assisted wrist training. J Electromyogr Kinesiol 2009, 19(4):639-650. [http://dx.doi.org/10.1016/j.jelekin.2008.04.002]

19. Hu XL, Tong KY, Song R, Zheng XJ, Leung WWF: A comparison between electromyography-driven robot and passive motion device on wrist rehabilitation for chronic stroke. Neurorehabil Neural Repair 2009, 23(8):837-846. [http://dx.doi.org/10.1177/1545968309338191] 
20. Sale P, Lombardi V, Franceschini M: Hand robotics rehabilitation: feasibility and preliminary results of a robotic treatment in patients with hemiparesis. Stroke Res Treat 2012, 2012:820931. [http://dx.doi.org/10.1155/2012/820931]

21. Chen M, Ho SK, Zhou HF, Pang PMK, Hu XL, Ng DTW, Tong KY: Interactive rehabilitation robot for hand function training. In ProC. IEEE International Conference on Rehabilitation Robotics ICORR. Kyoto, Japan; 2009:777-780

22. Turner M, Gomez D, Tremblay M, Cutkosky M: Preliminary tests of an arm-grounded haptic feedback device in telemanipulation. In Proc of the ASME Dynamic Systems and Control Division. Volume 64. Anaheim, CA; 1998:145-149.

23. Ertas $\mathrm{H}$, Hocaoglu E, Barkana DE, Patoglu V: Finger exoskeleton for treatment of tendon injuries. In Proc. IEEE International Conference on Rehabilitation Robotics ICORR. Kyoto, Japan; 2009:194-201.

24. Fuxiang Z: An Embedded Control Platform of a Continuous Passive Motion Machine for Injured Fingers. In Rehabilitation Robotics. Edited by Kommu SS. Vienna, Austria: I-Tech Education Publishing; 2007:579-606.

25. Vanoglio F, Luisa A, Garofali F, Mora C: Evaluation of the effectiveness of Gloreha (Hand Rehabilitation Glove) on hemiplegic patients. Pilot study. In XIII Congress of Italian Society of Neurorehabilitation, 18-20 April. Italy: Bari; 2013.

26. Parrinello I, Faletti S, Santus G: Use of a continuous passive motion device for hand rehabilitation: clinical trial on neurological patients. In 41 National Congress of Italian Society of Medicine and Physical Rehabilitation, 14-16 October. Rome, Italy; 2013.

27. Varalta V, Smania N, Geroin C, Fonte C, Gandolfi M, Picelli A, Munari D, lanes $P$, Montemezzi $G$, La Marchina E: Effects of passive rehabilitation of the upper limb with robotic device Gloreha on visual-spatial and attentive exploration capacities of patients with stroke issues. In XIII Congress of Italian Society of Neurorehabilitation, 18-20 April. Bari, Italy; 2013.

28. Ho NSK, Tong KY, Hu XL, Fung KL, Wei XJ, Rong W, Susanto EA: An EMG-driven exoskeleton hand robotic training device on chronic stroke subjects: task training system for stroke rehabilitation. IEEE Int Conf Rehabil Robot; Boston, MA 2011, 2011:5975340. [http://dx.doi. org/10.1109/ICORR.2011.5975340]

29. Schabowsky CN, Godfrey SB, Holley RJ, Lum PS, Development and pilot testing of HEXORR: hand EXOskeleton rehabilitation robot. $J$ Neuroeng Rehabil 2010, 7:36. [http://dx.doi.org/10.1186/1743-0003-7-36]

30. Kline T, Kamper D, Schmit B: Control system for pneumatically controlled glove to assist in grasp activities. In Proc. 9th International Conference on Rehabilitation Robotics ICORR. Chicago, IL; 2005:78-81.

31. Mulas M, Folgheraiter M, Gini G: An EMG-controlled exoskeleton for hand rehabilitation. In Proc. 9th International Conference on Rehabilitation Robotics ICORR. Chicago, IL; 2005:371-374.

32. Hesse S, Kuhlmann H, Wilk J, Tomelleri C, Kirker SGB: A new electromechanical trainer for sensorimotor rehabilitation of paralysed fingers: a case series in chronic and acute stroke patients. J Neuroeng Rehabil 2008, 5:21. [http://dx.doi.org/10.1186/ 1743-0003-5-21]

33. Rotella MF, Reuther KE, Hofmann CL, Hage EB, BuSha BF: An Orthotic Hand-Assistive Exoskeleton for Actuated Pinch and Grasp. In Bioengineering Conference, IEEE 35th Annual Northeast. Boston, MA; 2009:1-2. [http://dx.doi.org/10.1109/NEBC.2009.4967693]

34. Sarakoglou I, Tsagarakis NG, Caldwell DG: Occupational and physical therapy using a hand exoskeleton based exerciser. In Proc. IEEE/RSJ International Conference on Intelligent Robots and Systems (IROS). Volume 3. Sendai, Japan; 2004:2973-2978.

35. Tong KY, Ho SK, Pang PK, Hu XL, Tam WK, Fung KL, Wei XJ, Chen PN, Chen $M$ : An intention driven hand functions task training robotic system. In Conf Proc IEEE Eng Med Bio/ Soc. Volume 2010. Buenos Aires, Argentina; 2010:3406-3409. [http://dx.doi.org/10.1109/IEMBS.2010.5627930]

36. Wege A, Hommel G: Development and control of a hand exoskeleton for rehabilitation of hand injuries. In Intelligent Robots and Systems, (IROS 2005). 2005 IEEE/RSJ International Conference on. Edmonton, Canada; 2005:3046-3051.

37. Worsnopp TT, Peshkin MA, Colgate JE, Kamper DG: An Actuated Finger Exoskeleton for Hand Rehabilitation Following Stroke. In PrOC. IEEE 10th International Conference on Rehabilitation Robotics ICORR. Noordwijk, Netherlands; 2007:896-901.

38. Xing K, Xu Q, He J, Wang Y, Liu Z, Huang X: A wearable device for repetitive hand therapy. In Proc. 2nd IEEE RAS \& EMBS International Conference on Biomedical Robotics and Biomechatronics BioRob. Scottsdale, AZ; 2008:919-923.

39. Ellis MD, Sukal T, DeMott T, Dewald JPA: ACT 3D exercise targets gravity-induced discoordination and improves reaching work area in individuals with stroke. In Proc. IEEE 10th International Conference on Rehabilitation Robotics /CORR. Noordwijk, Netherlands; 2007:890-895.

40. Kahn LE, Lum PS, Rymer WZ, Reinkensmeyer DJ: Robot-assisted movement training for the stroke-impaired arm: Does it matter what the robot does? J Rehabil Res Dev 2006, 43(5):619-630.

41. Chang JJ, Tung WL, Wu WL, Huang MH, Su FC: Effects of robot-aided bilateral force-induced isokinetic arm training combined with conventional rehabilitation on arm motor function in patients with chronic stroke. Arch Phys Med Rehabil 2007, 88(10):1332-1338. [http://dx.doi.org/10.1016/j.apmr.2007.07.016]

42. Volpe BT, Krebs HI, Hogan N, OTR LE, Diels C, Aisen M: A novel approach to stroke rehabilitation: robot-aided sensorimotor stimulation. Neurology 2000, 54(10):1938-1944.

43. Rabadi M, Galgano M, Lynch D, Akerman M, Lesser M, Volpe B: A pilot study of activity-based therapy in the arm motor recovery post stroke: a randomized controlled trial. Clin Rehabil 2008, 22(12):1071-1082. [http://dx.doi.org/10.1177/0269215508095358]

44. Ju MS, Lin CCK, Lin DH, Hwang IS, Chen SM: A rehabilitation robot with force-position hybrid fuzzy controller: hybrid fuzzy control of rehabilitation robot. IEEE Trans Neural Syst Rehabil Eng 2005, 13(3):349-358. [http://dx.doi.org/10.1109/TNSRE.2005.847354]

45. Kiguchi K, Rahman MH, Sasaki M, Teramoto K: Development of a 3DOF mobile exoskeleton robot for human upper-limb motion assist. Robotics and Autonomous Systems 2008, 56(8):678-691. [http://www. sciencedirect.com/science/article/B6V16-4R8MDRP-1/2/ 7d307e7bbef3e5958a6960e3da652723]

46. Rosati G, Gallina P, Masiero S, Rossi A: Design of a new 5 d.o.f. wire-based robot for rehabilitation. In Proc. 9th International Conference on Rehabilitation Robotics ICORR. Chicago, IL; 2005:430-433.

47. Colombo R, Sterpi I, Mazzone A, Delconte C, Minuco G, Pisano F: Measuring changes of movement dynamics during robot-aided neurorehabilitation of stroke patients. IEEE Trans Neural Syst Rehabil Eng 2010, 18:75-85. [http://dx.doi.org/10.1109/TNSRE.2009.2028831]

48. Lum PS, Burgar CG, Shor PC, Majmundar M, der Loos, M V: Robot-assisted movement training compared with conventional therapy techniques for the rehabilitation of upper-limb motor function after stroke. Arch Phys Med Rehabil 2002, 83(7):952-959.

49. Lum PS, Burgar CG, der Loos, M V, Shor PC, Majmundar M, Yap R: MIME robotic device for upper-limb neurorehabilitation in subacute stroke subjects: A follow-up study. J Rehabil Res Dev 2006, 43(5):631-642.

50. Lum PS, Burgar CG, Shor PC: Evidence for improved muscle activation patterns after retraining of reaching movements with the MIME robotic system in subjects with post-stroke hemiparesis. IEEE Trans Neural Syst Rehabil Eng 2004, 12(2):186-194. [http://dx.doi.org/ 10.1109/TNSRE.2004.827225]

51. Moubarak S, Pham M, Pajdla T, Redarce T: Design Results of an Upper Extremity Exoskeleton. In Proc. 4th European Conference of the International Federation for Medical and Biological Engineering. Antwerp, Belgium; 2008.

52. Masiero S, Celia A, Rosati G, Armani M: Robotic-assisted rehabilitation of the upper limb after acute stroke. Arch Phys Med Rehabil 2007, 88(2):142-149. [http://dx.doi.org/10.1016/j.apmr.2006.10.032]

53. Masiero S, Armani M, Rosati G: Upper-limb robot-assisted therapy in rehabilitation of acute stroke patients: focused review and results of new randomized controlled trial. J Rehabil Res Dev 2011, 48(4):355-366.

54. Fazekas $G$, Horvath M, Troznai $T$, Toth A: Robot-mediated upper limb physiotherapy for patients with spastic hemiparesis: a preliminary study. J Rehabil Med 2007, 39(7):580-582. [http://www.ingentaconnect. com/content/mjl/sreh/2007/00000039/00000007/art00013]

55. Hesse S, Schulte-Tigges G, Konrad M, Bardeleben A, Werner C: Robot-assisted arm trainer for the passive and active practice of 
bilateral forearm and wrist movements in hemiparetic subjects. Arch Phys Med Rehabil 2003, 84(6):915-920.

56. Hesse S, Werner C, Pohl M, Rueckriem S, Mehrholz J, Lingnau ML: Computerized arm training improves the motor control of the severely affected arm after stroke: a single-blinded randomized trial in two centers. Stroke 2005, 36(9):1960-1966. [http://dx.doi.org/10. 1161/01.STR.0000177865.37334.ce]

57. Allington J, Spencer SJ, Klein J, Buell M, Reinkensmeyer DJ, Bobrow J: Supinator Extender (SUE): a pneumatically actuated robot for forearm/wrist rehabilitation after stroke. Conf ProC IEEE Eng Med Biol Soc 2011, 2011:1579-1582. [http://dx.doi.org/10.1109/IEMBS.2011. 6090459]

58. Cordo P, Lutsep H, Cordo L, Wright WG, Cacciatore T, Skoss R: Assisted movement with enhanced sensation (AMES): coupling motor and sensory to remediate motor deficits in chronic stroke patients. Neurorehabil Neural Repair 2009, 23:67-77. [http://dx.doi.org/10.1177/ 1545968308317437]

59. Koeneman EJ, Schultz RS, Wolf SL, Herring DE, Koeneman JB: A pneumatic muscle hand therapy device. Conf Proc IEEE Eng Med Biol Soc 2004, 4:2711-2713. [http://dx.doi.org/10.1109/IEMBS.2004.1403777]

60. Kutner NG, Zhang R, Butler AJ, Wolf SL, Alberts JL: Quality-of-life change associated with robotic-assisted therapy to improve hand motor function in patients with subacute stroke: a randomized clinical trial. Phys Ther 2010, 90(4):493-504. [http://dx.doi.org/10.2522/ ptj.20090160]

61. Rosenstein L, Ridgel AL, Thota A, Samame B, Alberts JL: Effects of combined robotic therapy and repetitive-task practice on upper-extremity function in a patient with chronic stroke. $\mathrm{Am} J$ Occup Ther 2008, 62:28-35.

62. Frick EM, Alberts JL: Combined use of repetitive task practice and an assistive robotic device in a patient with subacute stroke. Phys Ther 2006, 86(10):1378-1386. [http://dx.doi.org/10.2522/ptj. 20050149]

63. Johnson M, Wisneski K, Anderson J, Nathan D, Smith R: Development of ADLER: The activities of daily living exercise robot. In 1st IEEE/ RAS-EMBS Int. Conf. Biomedical Robotics and Biomechatronics, BioRob. Pisa, Italy; 2006:881-886.

64. Wisneski KJ, Johnson MJ: Quantifying kinematics of purposeful movements to real, imagined, or absent functional objects: implications for modelling trajectories for robot-assisted ADL tasks. J Neuroeng Rehabil 2007, 4:7. [http://dx.doi.org/10.1186/17430003-4-7]

65. Pignolo L, Dolce G, Basta G, Lucca LF, Serra S, Sannita WG: Upper limb rehabilitation after stroke: ARAMIS a "robo-mechatronic" innovative approach and prototype. In 4th IEEE RAS \& EMBS Int. Conf. Biomedical Robotics and Biomechatronics (BioRob). Rome, Italy; 2012:1410-1414. [http://ieeexplore.ieee.org/stamp/stamp.jsp? arnumber $=6290868]$

66. Coote S, Murphy B, Harwin W, Stokes E: The effect of the GENTLE/s robot-mediated therapy system on arm function after stroke. Clin Rehabil 2008, 22(5):395-405. [http://dx.doi.org/10.1177/ 0269215507085060]

67. Culmer PR, Jackson AE, Makower SG, Cozens JA, Levesley MC, Mon-Williams M, Bhakta B: A novel robotic system for quantifying arm kinematics and kinetics: description and evaluation in therapist-assisted passive arm movements post-stroke. J Neurosci Methods 2011, 197(2):259-269. [http://dx.doi.org/10.1016/j.jneumeth. 2011.03.004]

68. Kiguchi K, Kose Y, Hayashi Y: Task-oriented perception-assist for an upper-limb powerassist exoskeleton robot. In Proc. World Automation Congress (WAC). Kobe, Japan; 2010:1-6. [http://ieeexplore. ieee.org/xpls/abs_all.jsp?arnumber=5665314]

69. Frisoli A, Bergamasco M, Borelli L, Montagner A, Greco G, Procopio C, Carboncini M, Rossi B: Robotic assisted rehabilitation in virtual reality with the L-EXOS. In Proc. of 7th ICDVRAT with ArtAbilitation. Maia, Portugal; 2008:253-260.

70. Carignan C, Tang J, Roderick S, Naylor M: A Configuration-Space Approach to Controlling a Rehabilitation Arm Exoskeleton. In Proc IEEE 10th International Conference on Rehabilitation Robotics ICORR. Noordwijk, Netherlands; 2007:179-187.
71. Fluet GG, Qiu Q, Saleh S, Ramirez D, Adamovich S, Kelly D, Parikh H: Robot-assisted virtual rehabilitation (NJIT-RAVR) system for children with upper extremity hemiplegia. In Virtual Rehabilitation International Conference. Haifa, Israel; 2009:189-192.

72. Wolbrecht ET, Chan V, Reinkensmeyer DJ, Bobrow JE: Optimizing compliant, model-based robotic assistance to promote neurorehabilitation. IEEE Trans Neural Syst Rehabil Eng 2008, 16(3):286-297. [http://dx.doi.org/10.1109/TNSRE.2008.918389]

73. Housman SJ, Le V, Rahman T, Sanchez RJ, Reinkensmeyer DJ: ArmTraining with T-WREX After Chronic Stroke: Preliminary Results of a Randomized Controlled Trial. In Proc. IEEE 10th International Conference on Rehabilitation Robotics ICORR. Noordwijk, Netherlands; 2007:562-568.

74. Housman SJ, Scott KM, Reinkensmeyer DJ: A randomized controlled trial of gravity-supported, computer-enhanced arm exercise for individuals with severe hemiparesis. Neurorehabil Neural Repair 2009, 23(5):505-514. [http://dx.doi.org/10.1177/ 1545968308331148]

75. Sanchez RJ, Liu J, Rao S, Shah P, Smith R, Rahman T, Cramer SC, Bobrow JE, Reinkensmeyer $D J$ : Automating arm movement training following severe stroke: functional exercises with quantitative feedback in a gravity-reduced environment. IEEE Trans Neural Syst Rehabil Eng 2006, 14(3):378-389. [http://dx.doi.org/10.1109/TNSRE.2006.881553]

76. Gupta A, O'Malley M: Design of a haptic arm exoskeleton for training and rehabilitation. IEEE ASME Trans Mechatronics 2006, 11(3):280.

77. Lambercy O, Dovat L, Gassert R, Burdet E, Teo CL, Milner T: A haptic knob for rehabilitation of hand function. IEEE Trans Neural Syst Rehabil Eng 2007, 15(3):356-366. [http://dx.doi.org/10.1109/TNSRE.2007.903913]

78. Casadio M, Giannoni P, Morasso P, Sanguineti V: A proof of concept study for the integration of robot therapy with physiotherapy in the treatment of stroke patients. Clin Rehabil 2009, 23(3):217-228. [http://dx.doi.org/10.1177/0269215508096759]

79. Carpinella I, Cattaneo D, Abuarqub S, Ferrarin M: Robot-based rehabilitation of the upper limbs in multiple sclerosis: feasibility and preliminary results. J Rehabil Med 2009, 41(12):966-970. [http:// www.ingentaconnect.com/content/mjl/sreh/2009/00000041/ 00000012/art00004]

80. Casadio M, Sanguineti V, Solaro C, Morasso PG: A Haptic Robot Reveals the Adaptation Capability of Individuals with Multiple Sclerosis. Int J Rob Res 2007, 26(11-12):1225-1233.

81. Vergaro E, Squeri V, Brichetto G, Casadio M, Morasso P, Solaro C, Sanguineti $V$ : Adaptive robot training for the treatment of incoordination in Multiple Sclerosis. J Neuroeng Rehabil 2010, 7:37 [http://dx.doi.org/10.1186/1743-0003-7-37]

82. Denève A, Moughamir S, Afilal L, Zaytoon J: Control system design of a 3-DOF upper limbs rehabilitation robot. Comput Methods Programs Biomed 2008, 89(2):202-214. [http://dx.doi.org/10.1016/j. cmpb.2007.07.006]

83. Furuhashi Y, Nagasaki M, Aoki T, Morita Y, Ukai H, Matsui N: Development of rehabilitation support robot for personalized rehabilitation of upper limbs. In Proc. IEEE International Conference on Rehabilitation Robotics ICORR. Kyoto, Japan; 2009:787-792.

84. Mathai A, Qiu Q: Incorporating Haptic Effects Into Three-Dimensional Virtual Environments to Train the Hemiparetic Upper Extremity; 2009. [http://dx.doi.org/10.1109/TNSRE.2009.2028830]

85. Ozawa T, Kikuchi T, Fukushima K, Akai H, Fukuda T, Tanida S, Fujikawa T, Kano S, Furusho J: Initial clinical tests for assessment models of synergy movements of stroke patients using PLEMO system with sensor grip device. In Proc. IEEE International Conference on Rehabilitation Robotics ICORR. Kyoto, Japan; 2009:873-878.

86. Zhang H, Balasubramanian S, Wei R, Austin H, Buchanan S, Herman R, He J: RUPERT closed loop control design. Conf Proc IEEE Eng Med Biol Soc 2010, 2010:3686-3689. [http://dx.doi.org/10.1109/IEMBS.2010. 5627647]

87. Rosati G, Zanotto D, Secoli R, Rossi A: Design and control of two planar cable-driven robots for upper-limb neurorehabilitation. In Proc. IEEE International Conference on Rehabilitation Robotics ICORR. Kyoto, Japan; 2009:560-565.

88. Tanaka Y, Ishii M, Tsuji T, Imamura N: Modeling and evaluation of human motor skills in a virtual tennis task. Conf ProC IEEE Eng Med Biol Soc; Vancouver, Canada 2008, 2008:4190-4193. [http://dx.doi.org/10. 1109/IEMBS.2008.4650133] 
89. Xiu-Feng Z, Lin-Hong J, Li-Yun G: A novel robot neurorehabilitation for upper limb motion. Conf Proc IEEE Eng Med Biol Soc; Shanghai, China 2005, 5:5040-5043. [http://dx.doi.org/10.1109/IEMBS.2005.1615608]

90. Nef T, Guidali M, Klamroth-Marganska V, Riener R: ARMin - Exoskeleton Robot for Stroke Rehabilitation. In World Congress on Medical Physics and Biomedical Engineering, September 7-12. Volume 25/IX of IFMBE Proceedings. Edited by Dössel O, Schlegel WC. Munich, Germany: Springer; 2009:127-130.

91. Nef T, Quinter G, Müller R, Riener: Effects of arm training with the robotic device ARMin I in chronic stroke: three single cases. Neurodegener Dis 2009, 6(5-6):240-251. [http://dx.doi.org/10.1159/ 000262444]

92. Staubli P, Nef T, Klamroth-Marganska V, Riener R: Effects of intensive arm training with the rehabilitation robot ARMin II in chronic stroke patients: four single-cases. J Neuroeng Rehabil 2009, 6:46. [http://dx.doi.org/10.1186/1743-0003-6-46]

93. Gijbels D, Lamers I, Kerkhofs L, Alders G, Knippenberg E, Feys P: The Armeo Spring as training tool to improve upper limb functionality in multiple sclerosis: a pilot study. J Neuroeng Rehabil 2011, 8:5. [http://dx.doi.org/10.1186/1743-0003-8-5]

94. Loureiro RCV, Lamperd B, Collin C, Harwin WS: Reach \& grasp therapy: Effects of the Gentle/G System assessing sub-acute stroke whole-arm rehabilitation. In Proc. IEEE International Conference on Rehabilitation Robotics ICORR. Volume 2009. Kyoto; 2009:755-760.

95. Bovolenta F, Sale P, Dall'Armi V, Clerici P, Franceschini M: Robot-aided therapy for upper limbs in patients with stroke-related lesions. Brief report of a clinical experience. J Neuroeng Rehabil 2011, 8:18.

96. Treger I, Faran S, Ring H: Robot-assisted therapy for neuromuscular training of sub-acute stroke patients. A feasibility study. Eur J Phys Rehabil Med 2008, 44(4):431-435.

97. Perry JC, Rosen J, Burns S: Upper-limb powered exoskeleton design. Mechatronics, IEEE/ASME Trans 2007, 12(4):408-417

98. Hasegawa Y, Mikami Y, Watanabe K, Sankai Y: Five-fingered assistive hand with mechanical compliance of human finger. In IEEE Int. Conf. Robotics and Automation (ICRA). Pasadena, CA; 2008:718-724.

99. Rocon E, Belda-Lois JM, Ruiz AF, Manto M, Moreno JC, Pons JL: Design and validation of a rehabilitation robotic exoskeleton for tremor assessment and suppression. IEEE Trans Neural Syst Rehabil Eng 2007, 15(3):367-378. [http://dx.doi.org/10.1109/TNSRE.2007.903917]

100. Loureiro RCV, Belda-Lois JM, Lima ER, Pons JL, Sanchez-Lacuesta JJ, Harwin WS: Upper limb tremor suppression in ADL via an orthosis incorporating a controllable double viscous beam actuator. In Proc. 9th Int. Conf. on Rehabilitation Robotics ICORR. Chicago, IL; 2005:119-122.

101. Pedrocchi A, Ferrante S, Ambrosini E, Gandolla M, Casellato C, Schauer T, Klauer C, Pascual J, Vidaurre C, Gfoehler M, Reichenfelser W, Karner J, Micera S, Crema A, Molteni F, Rossini M, Palumbo G, Guanziroli E, Jedlitschka A, Hack M, Bulgheroni M, D Amico E, Schenk P, Zwicker S, Duschau-Wicke A, Miseikis J, Graber L, Ferrigno G: MUNDUS project: MUltimodal Neuroprosthesis for daily upper limb support. J Neuroeng Rehabil 2013, 10:66. [http://dx.doi.org/10.1186/1743-000310-66]

102. Dijkers MP, deBear PC, Erlandson RF, Kristy K, Geer DM, Nichols A: Patient and staff acceptance of robotic technology in occupational therapy: a pilot study. J Rehabil Res Dev 1991, 28(2):33-44.

103. Donnan GA, Fisher M, Macleod M, Davis SM: Stroke. Lancet 2008, 371 (9624):1612-1623. [http://dx.doi.org/10.1016/S0140-6736(08)60694-7]

104. Oda K, Isozumi S, Ohyama Y, Tamida K, Kikuchi T, Furusho J: Development of isokinetic and iso-contractile exercise machine MEM-MRB using MR brake. In Proc. IEEE Int. Conf. on Rehabilitation Robotics (ICORR). Kyoto, Japan; 2009:6-11.

105. Kikuchi T, Xinghao H, Fukushima K, Oda K, Furusho J, Inoue A: Quasi-3-DOF rehabilitation system for upper limbs: its force-feedback mechanism and software for rehabilitation. In Proc. IEEE 10th International Conference on Rehabilitation Robotics (ICORR). Noordwijk, Netherlands; 2007:24-27.

106. Sanchez R, Reinkensmeyer D, Shah P, Liu J, Rao S, Smith R, Cramer S, Rahman T, Bobrow J: Monitoring functional arm movement for home-based therapy after stroke. Conf Proc IEEE Eng Med Bio/ Soc; San Francisco, CA 2004, 7:4787-4790. [http://dx.doi.org/10.1109/IEMBS.2004. 1404325]
107. Krebs HI, Hogan N, Aisen ML, Volpe BT: Robot-aided neurorehabilitation. IEEE Trans Rehabil Eng 1998, 6:75-87.

108. Schoone M, van Os, P, Campagne A: Robot-mediated Active Rehabilitation (ACRE) A user trial. In Proc. IEEE 10th International Conference on Rehabilitation Robotics (ICORR). Noordwijk, Netherlands; 2007:477-481

109. Spencer SJ, Klein J, Minakata K, Le V, Bobrow JE, Reinkensmeyer DJ: A low cost parallel robot and trajectory optimization method for wrist and forearm rehabilitation using the Wii. In Proc. 2nd IEEE RAS \& EMBS International Conference on Biomedical Robotics and Biomechatronics (BioRob). Scottsdale, AZ; 2008:869-874.

110. Takaiwa M, Noritsugu T: Development of Wrist Rehabilitation Equipment Using Pneumatic Parallel Manipulator. In Proc. IEEE International Conference on Robotics and Automation (ICRA). Barcelona, Spain; 2005:2302-2307. [http://dx.doi.org/10.1109/ROBOT.2005. 1570456]

111. Rosati G, Gallina P, Masiero S: Design, implementation and clinical tests of a wire-based robot for neurorehabilitation. IEEE Trans Neural Syst Rehabil Eng 2007, 15(4):560-569. [http://dx.doi.org/10.1109/TNSRE. 2007.908560]

112. Beer RF, Naujokas C, Bachrach B, Mayhew D: Development and evaluation of a gravity compensated training environment for robotic rehabilitation of post-stroke reaching. In Proc. 2nd IEEE RAS \& EMBS International Conference on Biomedical Robotics and Biomechatronics (BioRob). Scottsdale, AZ; 2008:205-210.

113. Dovat L, Lambercy O, Gassert R, Maeder T, Milner T, Leong TC, Burdet E: HandCARE: a cable-actuated rehabilitation system to train hand function after stroke. IEEE Trans Neural Syst Rehabil Eng 2008, 16(6):582-591. [http://dx.doi.org/10.1109/TNSRE.2008. 2010347]

114. Kiguchi K, Iwami K, Yasuda M, Watanabe K, Fukuda T: An exoskeletal robot for human shoulder joint motion assist. Mechatronics, IEEE/ASME Transactions on 2003, 8:125-135.

115. Schiele $A$, van der Helm, FCT: Kinematic design to improve ergonomics in human machine interaction. IEEE Trans Neural Syst Rehabil Eng 2006, 14(4):456-469. [http://dx.doi.org/10.1109/TNSRE.2006. 881565]

116. Ren Y, Park HS, Zhang LQ: Developing a whole-arm exoskeleton robot with hand opening and closing mechanism for upper limb stroke rehabilitation. In Proc. IEEE International Conference on Rehabilitation Robotics (ICORR). Kyoto, Japan; 2009:761-765.

117. Fleischer C, Kondak K, Wege A, Kossyk I: Research on Exoskeletons at the TU Berlin. In Proceedings of German Workshop on Robotics, June 9-10. Braunschweig, Germany; 2009. [http://www.gwr09.org/download/ paper/gwr09_0043.pdf]

118. Klein J, Spencer S, Allington J, Bobrow JE, Reinkensmeyer DJ: Optimization of a parallel shoulder mechanism to achieve a high-force, low-mass, robotic-arm exoskeleton. Robotics, IEEE Trans 2010, 26(4):710-715

119. Gupta A, O'Malley M, Patoglu V, Burgar C: Design, control and performance of RiceWrist: a force feedback wrist exoskeleton for rehabilitation and training. Int $J$ Robot Res 2008, 27(2):233.

120. Burgar CG, Lum PS, Shor PC, der Loos, H F M V: Development of robots for rehabilitation therapy: the Palo Alto VA/Stanford experience. J Rehabil Res Dev 2000, 37(6):663-673. [http://www.rehab.research.va. gov/jour/00/37/6/pdf/burgar.pdf]

121. Amirabdollahian F, Loureiro R, Gradwell E, Collin C, Harwin W, Johnson $G$ : Multivariate analysis of the Fugl-Meyer outcome measures assessing the effectiveness of GENTLE/S robot-mediated stroke therapy. J Neuroeng Rehabil 2007, 4:4. [http://dx.doi.org/10.1186/17430003-4-4]

122. Van Der Linde R, Lammertse P: HapticMaster-a generic force controlled robot for human interaction. Industrial Robot: Int J 2003, 30(6):515-524.

123. Loureiro RCV, Harwin WS: Reach \& Grasp Therapy: Design and Control of a 9-DOF Robotic Neuro-rehabilitation System. In Proc IEEE 10th International Conference on Rehabilitation Robotics (ICORR). Noordwijk, Netherlands; 2007:757-763.

124. Mihelj M, Podobnik J, Munih M: HEnRiE - Haptic environment for reaching and grasping exercise. In Proc. 2nd IEEE RAS \& EMBS 
International Conference on Biomedical Robotics and Biomechatronics (BioRob). Scottsdale, AZ; 2008:907-912.

125. Toth A, Fazekas G, Arz G, Jurak M, Horvath M, Passive robotic movement therapy of the spastic hemiparetic arm with REHAROB: report of the first clinical test and the follow-up system improvement. In Proc. 9th International Conference on Rehabilitation Robotics (ICORR). Chicago, IL; 2005:127-130.

126. Culmer PR, Jackson AE, Makower S, Richardson R, Cozens JA, Levesley MC, Bhakta BB: A Control Strategy for Upper Limb Robotic Rehabilitation With a Dual Robot System. Mechatronics, IEEE/ASME Trans 2009, PP(99):1-11.

127. Morales R, Badesa FJ, García-Aracil N, Sabater JM: Pneumatic robotic systems for upper limb rehabilitation. Med Biol Eng Comput 2011, 49(10):1145-1156. [http://dx.doi.org/10.1007/s11517-011-0814-3]

128. Houtsma JA, Houten FJAMV: Virtual reality and a haptic master-slave set-up in post-stroke upper-limb rehabilitation. Proc Inst Mech Eng H 2006, 220(6):715-718.

129. Krebs HI, Hogan N, Durfee W, Herr H: Rehabilitation robotics, orthotics and prosthetics. In Textbook of Neural Repair and Rehabilitation(Selzer M, Clarke S, Cohen L, Duncan P, Gage F, eds.): Cambridge Univ Press; 2006.

130. Takahashi CD, Der-Yeghiaian L, Le V, Motiwala RR, Cramer SC: Robot-based hand motor therapy after stroke. Brain 2008 131(Pt 2):425-437. [http://dx.doi.org/10.1093/brain/awm311]

131. Vertechy R, Frisoli A, Dettori A, Solazzi M, Bergamasco M: Development of a new exoskeleton for upper limb rehabilitation. In Proc. IEEE International Conference on Rehabilitation Robotics (ICORR). Kyoto, Japan; 2009:188-193.

132. Micera S, Carrozza M, Guglielmelli E, Cappiello G, Zaccone F, Freschi C, Colombo R, Mazzone A, Delconte C, Pisano F, et al.: A simple robotic system for neurorehabilitation. Autonomous Robots 2005, 19(3):271-284

133. Krebs H, Ferraro M, Buerger S, Newbery M, Makiyama A, Sandmann M, Lynch D, Volpe B, Hogan N: Rehabilitation robotics: pilot trial of a spatial extension for MIT-Manus. J Neuroeng Rehabil 2004, 1:5. [http:// dx.doi.org/10.1186/1743-0003-1-5]

134. Casadio M, Sanguineti V, Morasso PG, Arrichiello V: Braccio di Ferro: a new haptic workstation for neuromotor rehabilitation. Technol Health Care 2006, 14(3):123-142.

135. Kikuchi T, Ozawa T, Akai H, Furusho J: "Hybrid-PLEMO", rehabilitation system for upper limbs with active / passive force feedback, and its application for facilitation techniques. In Proc. IEEE International Conference on Rehabilitation Robotics (ICORR). Kyoto, Japan; 2009:781-786

136. Reinkensmeyer DJ, Kahn LE, Averbuch M, McKenna-Cole A, Schmit BD, Rymer WZ: Understanding and treating arm movement impairment after chronic brain injury: progress with the ARM guide. J Rehabil Res Dev 2000, 37(6):653-662.

137. Lum P, Reinkensmeyer D, Mahoney R, Rymer WZ, Burgar C: Robotic devices for movement therapy after stroke: current status and challenges to clinical acceptance. Top Stroke Rehabil 2002, 8(4):40-53.

138. Krebs HI, Volpe BT, Williams D, Celestino J, Charles SK, Lynch D, Hogan N: Robot-aided neurorehabilitation: a robot for wrist rehabilitation. IEEE Trans Neural Syst Rehabil Eng 2007, 15(3):327-335. [http://dx.doi.org/ 10.1109/TNSRE.2007.903899]

139. Oblak J, Cikajlo I, Matjacic Z: Universal Haptic Drive: A Robot for Arm and Wrist Rehabilitation. IEEE Trans Neural Syst Rehabil Eng 2009. [http://dx.doi.org/10.1109/TNSRE.2009.2034162]

140. Caldwell DC, Tsagarakis N: "Soft" Exoskeletons for Upper and Lower Body Rehabilitation - Design, Control and Testing. Int J Humanoid Robot 2007, 4:1-24.

141. Pratt GA, Williamson MM: Series elastic actuators. In Intelligent Robots and Systems 95. 'Human Robot Interaction and Cooperative Robots', Proceedings. 1995 IEEE/RSJ International Conference on. Volume 1. Pittsburgh, PA; 1995:399-406.

142. Sulzer JS, Peshkin MA, Patton JL: Design of a Mobile, Inexpensive Device for Upper Extremity Rehabilitation at Home. In PrOc. IEEE 10th International Conference on Rehabilitation Robotics (ICORR). Noordwijk, Netherlands; 2007:933-937.

143. Vanderniepen I, Van Ham R, Van Damme M, Versluys R, Lefeber D: Orthopaedic rehabilitation: A powered elbow orthosis using compliant actuation. In Proc. IEEE International Conference on Rehabilitation Robotics (ICORR). Kyoto, Japan; 2009:172-177.

144. Rosati G, Cenci S, Boschetti G, Zanotto D, Masiero S: Design of a single-dof active hand orthosis for neurorehabilitation. In PrOc. IEEE International Conference on Rehabilitation Robotics (ICORR). Kyoto, Japan; 2009:161-166.

145. Wolbrecht ET, Leavitt J, Reinkensmeyer DJ, Bobrow JE: Control of a pneumatic orthosis for upper extremity stroke rehabilitation. Conf Proc IEEE Eng Med Bio/ Soc; New York, NY 2006, 1:2687-2693. [http://dx. doi.org/10.1109/IEMBS.2006.259941]

146. Sasaki D, Noritsugu T, Takaiwa M: Development of Active Support Splint driven by Pneumatic Soft Actuator (ASSIST). In Proc. IEEE International Conference on Robotics and Automation (ICRA). Barcelona, Spain; 2005:520-525

147. Lucas L, DiCicco M, Matsuoka Y: An EMG-controlled hand exoskeleton for natural pinching. J Robot Mechatronics 2004, 16:482-488.

148. Tsagarakis N, Caldwell D: Development and control of a "soft-actuated" exoskeleton for use in physiotherapy and training. Autonomous Robots 2003, 15:21-33.

149. Kobayashi H, Nozaki H: Development of muscle suit for supporting manual worker. In IEEE/RSJ International Conference on Intelligent Robots and Systems (IROS). San Diego, CA; 2007:1769-1774.

150. Chou CP, Hannaford B: Measurement and modeling of McKibben pneumatic artificial muscles. IEEE Trans Robot Automation 1996, 12:90-102.

151. Balasubramanian S, Wei R, Perez M, Shepard B, Koeneman E, Koeneman J, He J: RUPERT: An exoskeleton robot for assisting rehabilitation of arm functions. In Proc. Virtual Rehabilitation. Vancouver, Canada; 2008:163-167. [http://dx.doi.org/10.1109/ICVR.2008.4625154]

152. Umemura A, Saito Y, Fujisaki K: A study on power-assisted rehabilitation robot arms operated by patient with upper limb disabilities. In Proc. IEEE International Conference on Rehabilitation Robotics (ICORR). Kyoto, Japan; 2009:451-456.

153. Pylatiuk C, Kargov A, Gaiser I, Werner T, Schulz S, Bretthauer G: Design of a flexible fluidic actuation system for a hybrid elbow orthosis. In Proc. IEEE International Conference on Rehabilitation Robotics (ICORR). Kyoto, Japan; 2009:167-171.

154. Stienen A, Hekman E, Prange G, Jannink M, Aalsma A, van der Helm F van der Kooij H: Dampace: Design of an exoskeleton for force-coordination training in upper-extremity rehabilitation. $J$ Med Devices 2009, 3:031003.

155. Stienen AHA, Hekman EEG, ter Braak, H, Aalsma AMM, van der Helm FCT, van der Kooij $\mathrm{H}$ : Design of a rotational hydro-elastic actuator for an active upper-extremity rehabilitation exoskeleton. In Proc. 2nd IEEE RAS \& EMBS International Conference on Biomedical Robotics and Biomechatronics (BioRob). Scottsdale, AZ; 2008:881-888.

156. Scherer R, Pradhan S, Dellon B, Kim D, Klatzky R, Matsuoka Y: Characterization of multi-finger twist motion toward robotic rehabilitation. In Proc. IEEE International Conference on Rehabilitation Robotics (ICORR). Kyoto, Japan; 2009:812-817.

157. Winter $\mathrm{SH}$, Bouzit M: Use of Magnetorheological fluid in a force feedback glove. IEEE Trans Neural Syst Rehabil Eng 2007, 15:2-8. [http:// dx.doi.org/10.1109/TNSRE.2007.891401]

158. Khanicheh A, Mintzopoulos D, Weinberg B, Tzika AA, Mavroidis C: MR_CHIROD v.2: magnetic resonance compatible smart hand rehabilitation device for brain imaging. Neural Syst Rehabil Eng IEEE Trans 2008, 16:91-98.

159. Furusho J, Koyanagi K, Imada Y, Fujii Y, Nakanishi K, Domen K, Miyakoshi $K$, Ryu U, Takenaka S, Inoue A: A 3-D rehabilitation system for upper limbs developed in a 5-year NEDO project and its clinical testing. Chicago, IL; 2005:53-56.

160. Furusho J, Kikuchi T, Oda K, Ohyama Y, Morita T, Shichi N, Jin Y, Inoue A: A 6-DOF Rehabilitation Support System for Upper Limbs including Wrists "Robotherapist" with Physical Therapy. In Proc. IEEE 10th International Conference on Rehabilitation Robotics (ICORR). Noordwijk, Netherlands; 2007:304-309.

161. Hamid S, Hayek R: Role of electrical stimulation for rehabilitation and regeneration after spinal cord injury: an overview. Eur Spine $J$ 2008, 17(9):1256-1269. [http://dx.doi.org/10.1007/s00586-008-0729-3]

162. Takano Y, Haneda Y, Maeda T, Sakai Y, Matsuse H, Kawaguchi T, Tagawa $Y$, Shiba N: Increasing muscle strength and mass of thigh in 
elderly people with the hybrid-training method of electrical stimulation and volitional contraction. Tohoku J Exp Med 2010, 221:77-85

163. Freeman CT, Hughes AM, Burridge JH, Chappell PH, Lewin PL, Rogers E: A robotic workstation for stroke rehabilitation of the upper extremity using FES. Med Eng Phys 2009, 31(3):364-373. [http://dx.doi. org/10.1016/j.medengphy.2008.05.008]

164. Li R, Hu XL, Tong KY: Combined Electromyography(EMG)-driven system with functional electrical stimulation (FES) for poststroke rehabilitation. In Proc. 2nd IEEE RAS \& EMBS International Conference on Biomedical Robotics and Biomechatronics (BioRob). Scottsdale, AZ; 2008:642-646

165. Masia L, Krebs HI, Cappa P, Hogan N: Design, Characterization, and Impedance Limits of a Hand Robot. In Proc. IEEE 10th International Conference on Rehabilitation Robotics (ICORR). Noordwijk, Netherlands; 2007:1085-1089.

166. Gopura RARC, Kiguchi K, Li Y: SUEFUL-7: A 7DOF upper-limb exoskeleton robot with muscle-model-oriented EMG-based control. In IEEE/RSJ International Conference on Intelligent Robots and Systems (IROS). St. Louis, MO; 2009:1126-1131.

167. Nathan DE, Johnson MJ, McGuire J: Feasibility of integrating FES grasp assistance with a task-oriented robot-assisted therapy environment: A case study. In Proc. 2nd IEEE RAS \& EMBS International Conference on Biomedical Robotics and Biomechatronics (BioRob). Scottsdale, AZ; 2008:807-812.

168. Johnson GR, Carus DA, Parrini G, Marchese SS, Valeggi R: The design of a five-degree-of-freedom powered orthosis for the upper limb. Proc Inst Mech Eng H 2001, 215(3):275-284.

169. Rosen J, Brand M, Fuchs MB, Arcan M: A myosignal-based powered exoskeleton system. IEEE Trans Syst, Man and Cybernet, Part A 2001, 31(3):210-222.

170. Kiguchi K, Esaki R, Tsuruta T, Watanabe K, Fukuda T: An exoskeleton system for elbow joint motion rehabilitation. In Proc. IEEE/ASME International Conference on Advanced Intelligent Mechatronics (AIM). Volume 2. Port Island, Japan; 2003:1228-1233. vol.2.

171. Ögce F, Özyalçin H: Case study: A myoelectrically controlled shoulder-elbow orthosis for unrecovered brachial plexus injury. Prosthet Orthot Int 2000, 24(3):252-255.

172. Stein J, Narendran K, McBean J, Krebs K, Hughes R: Electromyography-controlled exoskeletal upper-limb-powered orthosis for exercise training after stroke. Am J Phys Med Rehabil 2007, 86(4):255-261. [http://dx.doi.org/10.1097/PHM.0b013e3180383cc5]

173. Stein J: e100 NeuroRobotic system. Expert Rev Med Devices 2009, 6:15-19. [http://dx.doi.org/10.1586/17434440.6.1.15]

174. Gopura RAR, Kiguchi K: A human forearm and wrist motion assist exoskeleton robot with EMG-based Fuzzy-neuro control. In Proc 2nd IEEE RAS \& EMBS International Conference on Biomedical Robotics and Biomechatronics (BioRob). Scottsdale, AZ; 2008:550-555.

175. Song R, Tong KY, Hu XL, Zheng XJ: Myoelectrically Controlled Robotic System That Provide Voluntary Mechanical Help for Persons after Stroke. In Proc. IEEE 10th International Conference on Rehabilitation Robotics (ICORR). Noordwijk, Netherlands; 2007:246-249.

176. Li Q, Wang D, Du Z, Song Y, Sun L: sEMG Based Control for 5 DOF Upper Limb Rehabilitation Robot System. In Proc. IEEE International Conference on Robotics and Biomimetics (ROBIO). Kunming, China; 2006:1305-1310.

177. Mayr A, Kofler M, Saltuari L: [ARMOR: an electromechanical robot for upper limb training following stroke. A prospective randomised controlled pilot study]. Handchir Mikrochir Plast Chir 2008, 40:66-73. [http://dx.doi.org/10.1055/s-2007-989425]

178. Kawasaki H, Ito S, Ishigure Y, Nishimoto Y, Aoki T, Mouri T, Sakaeda H, Abe M: Development of a Hand Motion Assist Robot for Rehabilitation Therapy by Patient Self-Motion Control. In Proc. IEEE 10th International Conference on Rehabilitation Robotics (ICORR). Noordwijk, Netherlands; 2007:234-240.

179. Ding M, Ueda J, Ogasawara T: Pinpointed muscle force control using a power-assisting device: System configuration and experiment. In Proc. 2nd IEEE RAS \& EMBS International Conference on Biomedical Robotics and Biomechatronics (BioRob). Scottsdale, AZ; 2008:181-186.

180. Lam P, Hebert D, Boger J, Lacheray H, Gardner D, Apkarian J, Mihailidis A: A haptic-robotic platform for upper-limb reaching stroke therapy: preliminary design and evaluation results. J Neuroeng Rehabil 2008 5:15. [http://dx.doi.org/10.1186/1743-0003-5-15]

181. Casellato C, Pedrocchi A, Zorzi G, Vernisse L, Ferrigno G, Nardocci N: EMG-based visual-haptic biofeedback: a tool to improve motor control in children with primary dystonia. IEEE Trans Neural Syst Rehabil Eng 2013, 21(3):474-480. [http://dx.doi.org/10.1109/TNSRE.2012. 2222445]

182. Takahashi Y, Terada T, Inoue K, Ito Y, Ikeda Y, Lee H, Komeda T: Haptic Device System for Upper Limb Motor and Cognitive Function Rehabilitation: Grip Movement Comparison between Normal Subjects and Stroke Patients. In Proc. IEEE 10th International Conference on Rehabilitation Robotics (ICORR). Noordwijk, Netherlands; 2007: 736-741.

183. Mali U, Munih M: HIFE-haptic interface for finger exercise. Mechatronics, IEEE/ASME Trans 2006, 11:93-102.

184. Bouzit M, Burdea G, Popescu G, Boian R: The Rutgers Master II-new design force-feedback glove. Mechatronics, IEEE/ASME Trans 2002, 7(2):256-263.

185. Popescu VG, Burdea GC, Bouzit M, Hentz VR: A virtual-reality-based telerehabilitation system with force feedback. IEEE Trans Inf Technol Biomed 2000, 4:45-51.

186. Jack D, Boian R, Merians AS, Tremaine M, Burdea GC, Adamovich SV, Recce $M$, Poizner H: Virtual reality-enhanced stroke rehabilitation. Neural Syst Rehabil Eng, IEEE Trans 2001, 9(3):308-318.

187. Adamovich SV, Merians AS, Boian R, Lewis JA, Tremaine M, Burdea GS, Recce M, Poizner H: A virtual reality-based exercise system for hand rehabilitation post-stroke. Presence: Teleoperators \& Virtual Environ 2005, 14(2):161-174.

188. Brewer BR, Klatzky R, Matsuoka Y: Visual feedback distortion in a robotic environment for hand rehabilitation. Brain Res Bull 2008 75(6):804-813. [http://dx.doi.org/10.1016/j.brainresbull.2008.01.006]

189. Xydas EG, Louca LS: Design and Development of a Haptic Peg-Board Exercise for the Rehabilitation of People with Multiple Sclerosis. In Proc. IEEE 10th International Conference on Rehabilitation Robotics (ICORR) Noordwijk, Netherlands; 2007:906-916.

190. Palsbo SE, Hood-Szivek P: Effect of robotic-assisted three-dimensional repetitive motion to improve hand motor function and control in children with handwriting deficits: a nonrandomized phase 2 device trial. Am J Occup Ther 2012, 66(6):682-690. [http://dx.doi.org/10.5014/ajot.2012.004556]

191. Adamovich S, Fluet GG, Merians AS, Mathai A, Qiu Q: Recovery of hand function in virtual reality: Training hemiparetic hand and arm together or separately. Conf Proc IEEE Eng Med Biol Soc; Vancouver Canada 2008, 2008:3475-3478. [http://dx.doi.org/10.1109//EMBS.2008. 4649954]

192. Adamovich SV, Fluet GG, Mathai A, Qiu Q, Lewis J, Merians AS: Design of a complex virtual reality simulation to train finger motion for persons with hemiparesis: a proof of concept study. J Neuroeng Rehabil 2009, 6:28. [http://dx.doi.org/10.1186/1743-0003-6-28]

193. Marchal-Crespo L, Reinkensmeyer DJ: Review of control strategies for robotic movement training after neurologic injury. J Neuroeng Rehabil 2009, 6:20. [http://dx.doi.org/10.1186/1743-0003-6-20]

194. Patton JL, Stoykov ME, Kovic M, Mussa-Ivaldi FA: Evaluation of robotic training forces that either enhance or reduce error in chronic hemiparetic stroke survivors. Exp Brain Res 2006, 168(3):368-383. [http://dx.doi.org/10.1007/s00221-005-0097-8]

195. Hogan N: Impedance control: an approach to manipulation. Part 1 Theory. J Dynamic Syst, Meas Control 1985, 107:1-7.

196. Ott C, Mukherjee R, Nakamura Y: Unified Impedance and Admittance Control. In Proc. IEEE Int Conf Robotics and Automation (ICRA). Anchorage, AK; 2010:554-561

197. Frisoli A, Borelli L, Montagner A, Marcheschi S, Procopio C, Salsedo F, Bergamasco M, Carboncini MC, Tolaini M, Rossi B: Arm rehabilitation with a robotic exoskeleleton in Virtual Reality. In Proc. IEEE 10th International Conference on Rehabilitation Robotics (ICORR). Noordwijk, Netherlands; 2007:631-642.

198. Lo AC: Clinical designs of recent robot rehabilitation trials. Am J Phys Med Rehabil 2012, 91 (11 Suppl 3):S204-S216. [http://dx.doi.org/10.1097/ PHM.0b013e31826bcfa3] 
199. Kwakkel G, Kollen BJ, Krebs HI: Effects of robot-assisted therapy on upper limb recovery after stroke: a systematic review. Neurorehabil Neural Repair 2008, 22(2):111-121.

200. Mehrholz J, Platz T, Kugler J, Pohl M: Electromechanical and robot-assisted arm training for improving arm function and activities of daily living after stroke. Cochrane Database Syst Rev 2008 , 4:CD006876. [http://onlinelibrary.wiley.com/doi/10.1002/14651858. CD006876.pub2/full]

201. Timmermans AAA, Seelen HAM, Willmann RD, Kingma $H$ : Technology-assisted training of arm-hand skills in stroke: concepts on reacquisition of motor control and therapist guidelines for rehabilitation technology design. J Neuroeng Rehabil 2009, 6:1 . [http://dx.doi.org/10.1186/1743-0003-6-1]

202. Waldner A, Tomelleri C, Hesse S: Transfer of scientific concepts to clinical practice: recent robot-assisted training studies. Funct Neurol 2009, 24(4):173-177.

203. Lo AC, Guarino PD, Richards LG, Haselkorn JK, Wittenberg GF, Federman DG, Ringer RJ, Wagner TH, Krebs HI, Volpe BT, Bever CTJr, Bravata DM, Duncan PW, Corn BH, Maffucci AD, Nadeau SE, Conroy SS, Powell JM, Huang GD, Peduzzi P: Robot-assisted therapy for long-term upper-limb impairment after stroke. N Engl J Med 2010, 362(19):1772-1783. [http://dx.doi.org/10.1056/NEJMoa0911341]

204. Brochard S, Robertson J, Médée B, Rémy-Néris O: What's new in new technologies for upper extremity rehabilitation? Curr Opin Neurol 2010, 23(6):683-687. [http://dx.doi.org/10.1097/WCO. ob013e32833f61ce]

205. Reinkensmeyer DJ, Boninger ML: Technologies and combination therapies for enhancing movement training for people with a disability. J Neuroeng Rehabil 2012, 9:17. [http://dx.doi.org/10.1186/ 1743-0003-9-17]

doi:10.1186/1743-0003-11-3

Cite this article as: Maciejasz et al.: A survey on robotic devices for upper limb rehabilitation. Journal of NeuroEngineering and Rehabilitation 2014 11:3.

\section{Submit your next manuscript to BioMed Central} and take full advantage of:

- Convenient online submission

- Thorough peer review

- No space constraints or color figure charges

- Immediate publication on acceptance

- Inclusion in PubMed, CAS, Scopus and Google Scholar

- Research which is freely available for redistribution

Submit your manuscript at www.biomedcentral.com/submit
( Biomed Central 